\title{
Bifurcations and Dynamics of the Rb-E2F Pathway Involving miR449
}

\author{
Lingling $\mathrm{Li}^{1}$ and Jianwei Shen ${ }^{2}$ \\ ${ }^{1}$ Department of Basic Courses, Guangdong Polytechnic College, Zhaoqing 526100, China \\ ${ }^{2}$ Institute of Applied Mathematics, Xuchang University, Xuchang, Henan 461000, China \\ Correspondence should be addressed to Jianwei Shen; xcjwshen@gmail.com
}

Received 23 February 2017; Revised 19 April 2017; Accepted 14 May 2017; Published 19 October 2017

Academic Editor: Shanmugam Lakshmanan

Copyright (c) 2017 Lingling Li and Jianwei Shen. This is an open access article distributed under the Creative Commons Attribution License, which permits unrestricted use, distribution, and reproduction in any medium, provided the original work is properly cited.

\begin{abstract}
We focused on the gene regulative network involving Rb-E2F pathway and microRNAs (miR449) and studied the influence of time delay on the dynamical behaviors of Rb-E2F pathway by using Hopf bifurcation theory. It is shown that under certain assumptions the steady state of the delay model is asymptotically stable for all delay values; there is a critical value under another set of conditions; the steady state is stable when the time delay is less than the critical value, while the steady state is changed to be unstable when the time delay is greater than the critical value. Thus, Hopf bifurcation appears at the steady state when the delay passes through the critical value. Numerical simulations were presented to illustrate the theoretical results.
\end{abstract}

\section{Introduction}

In the last few years, an increasing number of noncoding RNA (or microRNA) have been discovered to play central regulatory roles in gene regulation processes of prokaryotes and eukaryotes [1]. It has become evident that miRNAs regulate a variety of biological processes, and their expression is often deregulated in human malignancy. On one hand, miRNAs play roles in tumorigenesis by modulating oncogenic and tumor suppressor pathways. On the other hand, the expressions of miRNAs can be regulated by several oncogenic or tumor suppressor transcription factors [2]. In this paper, we focus on miR449, which can induce cell senescence and apoptosis and act as a tumor suppressor through regulating $\mathrm{Rb} / \mathrm{E} 2 \mathrm{~F}$ activity $[2,3]$. In recent years, large numbers of researches have focused on the mechanisms controlling cellular proliferation associated with human cancer regulated by Rb-E2F pathway experimentally [4-7]. Rb and E2F proteins play important roles in the regulation of cell division, cell growth, and programmed cell death by controlling the expressions of genes involved in these processes, which are best known for their regulation of the cell cycle at the G1/S transition [8]. As the first identified tumor suppressor gene [9], $\mathrm{Rb}$ is recognized to play a fundamental role in a signaling pathway that controls cell proliferation [10]. Rb regulates the transcription of genes that are essential for DNA replication and cell cycle progression by binding and inhibiting E2F transcription factors [11]. In the Rb-E2F pathway including negative feedback loops involving miR449, miR449 provides a twofold safety mechanism to avoid excessive E2F-induced proliferation by cell cycle arrest and apoptosis [12]. Mathematical models have been established to explain the nonlinear dynamical behaviors of the Rb-E2F pathway [12-14], which mainly concentrate on the stability and bifurcation of the deterministic systems, but not taking into account the effects of time delay.

Time delay is one of the most important characteristics of gene regulation. In many cases, a gene regulates the expression of another gene by its products (RNAs or proteins). Since it takes time to generate those products and different processes need different amounts of time, time delayed regulation is ubiquitous in cellular processes [15]. Time-delay system is also called system with aftereffect or hereditary system $[16,17]$, and it is common in mathematical biology, such as population dynamics, the chemostat, neural network, blood cell maturation, transcriptional regulator dynamics, virus dynamics, and genetic network [18-23]. Recent papers have demonstrated that complex dynamical behaviors can arise 


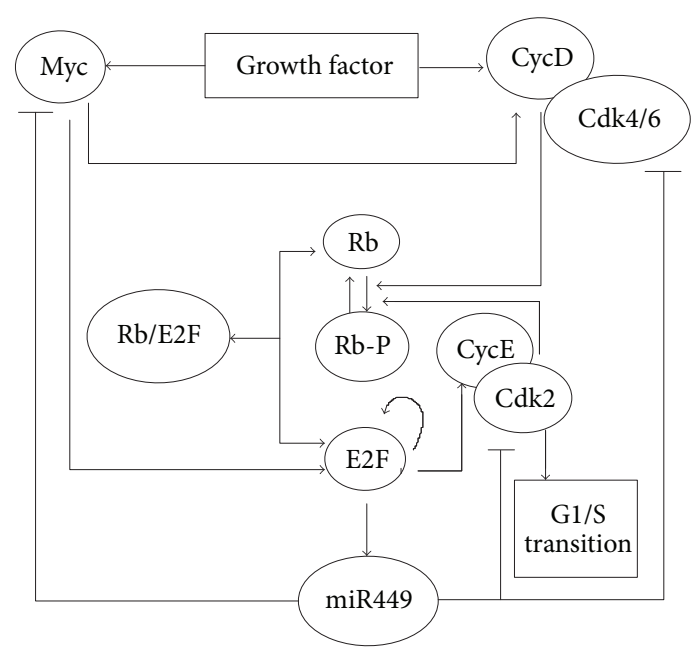

FIGURE 1: Rb-E2F pathway mediated by miR449.

as a consequence of time delays in biological systems. For example, in gene regulatory networks, time delays may lead to oscillations in protein levels and existing oscillations may become more robust $[24,25]$. Oscillatory cellular dynamics, in particular periodic oscillation, plays an important role in maintaining homeostasis of living organisms. In addition, taking into account time delays in models of gene regulatory networks is often essential to capture the whole range of dynamic behaviors. For example, in experiments, a single self-repressed gene has been observed to display oscillatory behavior, but which cannot be deduced by models that ignore time delays. However, this oscillatory behavior is reproduced by a mathematical model including time delays. In addition, theoretical analysis that ignored time delays led to the erroneous conclusion that oscillations were not possible for this single gene $[18,26]$. Many kinds of methods have been introduced to infer time delayed gene regulatory network [27-31]. Here, we mainly use the local linearization approach to analyze the nonlinear system.

In this paper, firstly, we will introduce $\mathrm{Rb}-\mathrm{E} 2 \mathrm{~F}$ pathway network involving miR449 modeled by time delayed differential equations. Secondly, we will study the dynamical behaviors of the model and derive sufficient conditions of the oscillation by using Hopf bifurcation theory. Particularly, we will also prove that there are periodic solutions under certain conditions. Finally, numerical simulations will be showed to illustrate the theoretical results.

\section{A Simple Gene Regulatory Network Mediated by miR449}

2.1. A Simple Mathematical Model of Gene Regulation with a Delayed Negative Feedback Loop. A simplified model (Figure 1) of the Rb-E2F pathway begins with growth signals activating $\mathrm{CycD}$. Initially, E2F is bound to and repressed by $\mathrm{Rb}$, a tumor suppressor protein that is dysfunctional in several major cancers. $\mathrm{CycD}$ represses the repressor $\mathrm{Rb}$ and allows E2F to be turned on. Myc also induces E2F transcription. Subsequently, E2F activates the transcription of CycE, which forms a complex with $\mathrm{Cdk} 2$ to further remove $\mathrm{Rb}$ repression, establishing a positive feedback loop. E2F also activates its own transcription, constituting another positive feedback loop. An interesting addition to the regulatory mechanism of Rb-E2F network is the recent discovery that miR449 modulates E2F activity. It has been demonstrated that E2F strongly upregulates the expression of miR449. In turn, E2F is inhibited by miR449 through regulating different transcripts. On one hand, miR449 directly affects level of its target transcript Myc and therefore lowers E2F concentration. On the other hand, miR449 directly affects E2F inducer Cdk6 and $\mathrm{CycE}$, thus forming negative feedback loops $[12,14]$. Yao et al. [14] provided a mathematical model in the absence of miR449 and indicated that the Rb-E2F pathway acts as a bistable switch to convert signal inputs into all-or-none E2F responses. Yan et al. [12] gave another mathematical model and further investigated the stabilities and bifurcations of E2F, $\mathrm{CycE}$, and miR449 in the participation of miR449. Our main work in this paper is considering the effects of time delays on the dynamic behaviors of the model including miR449. When time delays are taken into account, the time delayed differential equation model of the network including miR449 is described by the following system:

$$
\begin{aligned}
& \frac{d x_{1}}{d t}=k_{11} \frac{x_{3}}{k_{12}+x_{3}} \frac{x_{1}}{k_{13}+x_{1}}+k_{14} \frac{x_{3}}{k_{15}+x_{3}} \\
& +k_{16} x_{4} \frac{x_{8}}{k_{17}+x_{8}}+k_{18} x_{5} \frac{x_{8}}{k_{19}+x_{8}}-k_{110} x_{1} \\
& -k_{111} x_{6} x_{1} \\
& \frac{d x_{2}}{d t}=k_{21} \frac{x_{1}}{k_{22}+x_{1}}-k_{23} x_{2}-k_{24} x_{2} \frac{x_{3}}{k_{25}+x_{3}} \\
& -k_{26} x_{2} \frac{x_{4}}{k_{27}+x_{4}}-k_{28} x_{2} \frac{x_{5}}{k_{29}+x_{5}} \text {, } \\
& \frac{d x_{3}}{d t}=k_{31} \frac{S}{k_{32}+S}-k_{33} x_{3}-k_{34} x_{2}\left(t-\tau_{1}\right) \frac{x_{3}}{k_{35}+x_{3}} \text {, } \\
& \frac{d x_{4}}{d t}=k_{41} \frac{S}{k_{42}+S}+k_{43} \frac{x_{3}}{k_{44}+x_{3}}-k_{45} x_{4} \\
& -k_{46} x_{2}\left(t-\tau_{2}\right) \frac{x_{4}}{k_{47}+x_{4}} \\
& \frac{d x_{5}}{d t}=k_{51} \frac{x_{1}}{k_{52}+x_{1}}-k_{53} x_{5}-k_{54} x_{2}\left(t-\tau_{3}\right) \frac{x_{5}}{k_{55}+x_{5}} \text {, } \\
& \frac{d x_{6}}{d t}=k_{61}+k_{62} \frac{x_{7}}{k_{63}+x_{7}}-k_{64} x_{6} x_{1}-k_{65} x_{4} \frac{x_{8}}{k_{78}+x_{8}} \\
& -k_{67} x_{5} \frac{x_{6}}{k_{68}+x_{6}}-k_{69} x_{6} \text {, } \\
& \frac{d x_{7}}{d t}=k_{71} x_{4} \frac{x_{6}}{k_{72}+x_{6}}+k_{73} x_{5} \frac{x_{6}}{k_{74}+x_{6}} \\
& +k_{75} x_{4} \frac{x_{8}}{k_{76}+x_{8}}+k_{77} x_{5} \frac{x_{8}}{k_{78}+x_{8}} \\
& -k_{79} \frac{x_{7}}{k_{710}+x_{7}}-k_{711} x_{7} \text {, }
\end{aligned}
$$




$$
\begin{aligned}
\frac{d x_{8}}{d t}= & k_{81} x_{6} x_{1}-k_{82} x_{4} \frac{x_{8}}{k_{83}+x_{8}}-k_{84} x_{5} \frac{x_{8}}{k_{85}+x_{8}} \\
& -k_{86} x_{8} .
\end{aligned}
$$

\begin{tabular}{|c|c|}
\hline Rate constant & Value \\
\hline$k_{11}$ & 0.4 \\
\hline$k_{12}$ & 0.15 \\
\hline$k_{13}$ & 0.15 \\
\hline$k_{14}$ & 0.003 \\
\hline$k_{15}$ & 0.15 \\
\hline$k_{16}$ & 18 \\
\hline$k_{17}$ & 0.92 \\
\hline$k_{18}$ & 18 \\
\hline$k_{19}$ & 0.92 \\
\hline$k_{110}$ & 0.25 \\
\hline$k_{111}$ & 180 \\
\hline$k_{21}$ & 1.4 \\
\hline$k_{22}$ & 0.15 \\
\hline$k_{23}$ & 0.02 \\
\hline$k_{24}$ & 0.6 \\
\hline$k_{25}$ & 0.15 \\
\hline$k_{26}$ & 1 \\
\hline$k_{27}$ & 0.92 \\
\hline$k_{28}$ & 0.7 \\
\hline$k_{29}$ & 0.92 \\
\hline$k_{31}$ & 1 \\
\hline$k_{32}$ & 0.5 \\
\hline$k_{33}$ & 0.7 \\
\hline$k_{34}$ & 0.6 \\
\hline$k_{35}$ & 0.15 \\
\hline$k_{41}$ & 0.45 \\
\hline$k_{42}$ & 0.5 \\
\hline$k_{43}$ & 0.03 \\
\hline$k_{44}$ & 0.15 \\
\hline$k_{45}$ & 1.5 \\
\hline$k_{46}$ & 1 \\
\hline$k_{47}$ & 0.92 \\
\hline$k_{51}$ & 0.35 \\
\hline$k_{52}$ & 0.15 \\
\hline$k_{53}$ & 1.5 \\
\hline$k_{54}$ & 0.7 \\
\hline$k_{55}$ & 0.92 \\
\hline$k_{61}$ & 0.18 \\
\hline$k_{62}$ & 3.6 \\
\hline$k_{63}$ & 0.01 \\
\hline$k_{64}$ & 180 \\
\hline$k_{65}$ & 18 \\
\hline$k_{66}$ & 0.92 \\
\hline$k_{67}$ & 18 \\
\hline$k_{68}$ & 0.92 \\
\hline$k_{69}$ & 0.06 \\
\hline$k_{71}$ & 18 \\
\hline$k_{72}$ & 0.92 \\
\hline$k_{73}$ & 18 \\
\hline
\end{tabular}

TABLE 1: Parameters for the model. 
TABle 1: Continued.

\begin{tabular}{lc}
\hline Rate constant & Value \\
\hline$k_{74}$ & 0.92 \\
$k_{75}$ & 18 \\
$k_{76}$ & 0.92 \\
$k_{77}$ & 18 \\
$k_{78}$ & 0.92 \\
$k_{79}$ & 3.6 \\
$k_{710}$ & 0.01 \\
$k_{711}$ & 0.06 \\
$k_{81}$ & 180 \\
$k_{82}$ & 18 \\
$k_{83}$ & 0.92 \\
$k_{84}$ & 18 \\
$k_{85}$ & 0.92 \\
$k_{86}$ & 0.03 \\
\hline Note. The descriptions of the parameters in Table 1 are shown in $[13,15]$.
\end{tabular}

$$
\begin{aligned}
& -\frac{k_{14} x_{30}}{\left(k_{15}+x_{30}\right)^{2}} \\
& a_{14}=\frac{k_{16} x_{80}}{k_{17}+x_{80}}, \\
& a_{15}=\frac{k_{18} x_{80}}{k_{19}+x_{80}}, \\
& a_{16}=-k_{111} x_{10} \text {, } \\
& a_{18}=\frac{k_{16} x_{40}}{k_{17}+x_{80}}-\frac{k_{16} x_{40} x_{80}}{\left(k_{17}+x_{80}\right)^{2}}+\frac{k_{18}}{k_{50}} k_{19}+x_{80} \\
& -\frac{k_{18} x_{50} x_{80}}{\left(k_{19}+x_{80}\right)^{2}} \\
& a_{21}=\frac{k_{21}}{k_{22}+x_{10}}-\frac{k_{21} x_{10}}{\left(k_{22}+x_{10}\right)^{2}} \text {, } \\
& a_{22}=-k_{23}-\frac{k_{24} x_{30}}{k_{25}+x_{30}}-\frac{k_{26} x_{40}}{k_{27}+x_{40}}-\frac{k_{28} x_{50}}{k_{29}+x_{50}} \text {, } \\
& a_{23}=-\frac{k_{24} x_{20}}{k_{25}+x_{30}}+\frac{k_{24} x_{20} x_{30}}{\left(k_{25}+x_{30}\right)^{2}} \text {, } \\
& a_{24}=-\frac{k_{26} x_{20}}{k_{27}+x_{40}}+\frac{k_{26} x_{20} x_{40}}{\left(k_{27}+x_{40}\right)^{2}}, \\
& a_{25}=-\frac{k_{28} x_{20}}{k_{29}+x_{50}}+\frac{k_{28} x_{20} x_{50}}{\left(k_{29}+x_{50}\right)^{2}} \text {, } \\
& a_{33}=-k_{33}-\frac{k_{34} x_{20}}{k_{35}+x_{30}}+\frac{k_{34} x_{20} x_{30}}{\left(k_{35}+x_{30}\right)^{2}}, \\
& a_{43}=\frac{k_{43}}{k_{44}+x_{30}}-\frac{k_{43} x_{30}}{\left(k_{44}+x_{30}\right)^{2}},
\end{aligned}
$$

$$
\begin{aligned}
a_{44}= & -k_{45}-\frac{k_{46} x_{20}}{k_{47}+x_{40}}+\frac{k_{46} x_{20} x_{40}}{\left(k_{47}+x_{40}\right)^{2}}, \\
a_{51}= & \frac{k_{51}}{k_{52}+x_{10}}-\frac{k_{51} x_{10}}{\left(k_{52}+x_{10}\right)^{2}}, \\
a_{53}= & -k_{53}-\frac{k_{54} x_{20}}{k_{55}+x_{50}}+\frac{k_{54} x_{20} x_{50}}{\left(k_{55}+x_{50}\right)^{2}}, \\
a_{61}= & -k_{64} x_{60}, \\
a_{64}= & -\frac{k_{65} x_{60}}{k_{66}+x_{60}}, \\
a_{65}= & -\frac{k_{67} x_{60}}{k_{68}+x_{60}}, \\
a_{66}= & -k_{64} x_{10}-\frac{k_{65} x_{40}}{k_{66}+x_{60}}+\frac{k_{65} x_{40} x_{60}}{\left(k_{66}+x_{60}\right)^{2}}-\frac{k_{67} x_{50}}{k_{68}+x_{60}} \\
& +\frac{k_{67} x_{50} x_{60}}{\left(k_{68}+x_{60}\right)^{2}}-k_{69},
\end{aligned}
$$$$
a_{67}=\frac{k_{62}}{k_{63}+x_{70}}-\frac{k_{62} x_{70}}{\left(k_{63}+x_{70}\right)^{2}},
$$$$
a_{74}=\frac{k_{71} x_{60}}{k_{72}+x_{60}}+\frac{k_{75} x_{80}}{k_{76}+x_{80}},
$$$$
a_{75}=\frac{k_{73} x_{60}}{k_{74}+x_{60}}+\frac{k_{77} x_{80}}{k_{78}+x_{80}},
$$$$
a_{76}=\frac{k_{71} x_{40}}{k_{72}+x_{60}}-\frac{k_{71} x_{40} x_{60}}{\left(k_{72}+x_{60}\right)^{2}}+\frac{k_{73} x_{50}}{k_{74}+x_{60}}
$$$$
-\frac{k_{73} x_{50} x_{60}}{\left(k_{74}+x_{60}\right)^{2}}
$$$$
a_{77}=-\frac{k_{79}}{k_{710}+x_{70}}+\frac{k_{79} x_{70}}{\left(k_{710}+x_{70}\right)^{2}}-k_{711} \text {, }
$$$$
a_{78}=\frac{k_{75} x_{40}}{k_{76}+x_{80}}-\frac{k_{75} x_{40} x_{80}}{\left(k_{76}+x_{80}\right)^{2}}+\frac{k_{77} x_{50}}{k_{78}+x_{80}}
$$$$
-\frac{k_{77} x_{50} x_{80}}{\left(k_{78}+x_{80}\right)^{2}}
$$$$
a_{81}=k_{81} x_{60} \text {, }
$$$$
a_{84}=-\frac{k_{82} x_{80}}{k_{83}+x_{80}},
$$$$
a_{85}=-\frac{k_{84} x_{80}}{k_{85}+x_{80}},
$$$$
a_{86}=k_{81} x_{10} \text {, }
$$$$
a_{88}=-\frac{k_{82} x_{40}}{k_{83}+x_{80}}+\frac{k_{82} x_{40} x_{80}}{\left(k_{83}+x_{80}\right)^{2}}-\frac{k_{84} x_{50}}{k_{85}+x_{80}}
$$ 


$$
\begin{gathered}
+\frac{k_{84} x_{50} x_{80}}{\left(k_{85}+x_{80}\right)^{2}}-k_{86}, \\
b_{32}=-\frac{k_{34} x_{30}}{k_{35}+x_{30}}, \\
b_{42}=-\frac{k_{46} x_{40}}{k_{47}+x_{40}}, \\
b_{52}=-\frac{k_{54} x_{50}}{k_{55}+x_{50}} .
\end{gathered}
$$

Then we can obtain the characteristic equation of (2) at the equilibrium $\left(x_{10}, x_{20}, x_{30}, x_{40}, x_{50}, x_{60}, x_{70}, x_{80}\right)$ as follows:

$$
\left|\lambda I-A_{0}-B_{0} e^{-\lambda \tau}\right|=0,
$$

where $I$ is the $8 * 8$ identity matrix, and the characteristic equation (5) has the following form:

$$
\begin{aligned}
\lambda^{8} & +A_{1} \lambda^{7}+A_{2} \lambda^{6}+A_{3} \lambda^{5}+A_{4} \lambda^{4}+A_{5} \lambda^{3}+A_{6} \lambda^{2} \\
& +A_{7} \lambda+A_{8}+\left(B_{1} \lambda^{6}+B_{2} \lambda^{5}+B_{3} \lambda^{4}+B_{4} \lambda^{3}+B_{5} \lambda^{2}\right. \\
& \left.+B_{6} \lambda+B_{7}\right) e^{-\lambda \tau_{1}}+\left(C_{1} \lambda^{6}+C_{2} \lambda^{5}+C_{3} \lambda^{4}+C_{4} \lambda^{3}\right. \\
& \left.+C_{5} \lambda^{2}+C_{6} \lambda+C_{7}\right) e^{-\lambda \tau_{2}}+\left(E_{1} \lambda^{6}+E_{2} \lambda^{5}+E_{3} \lambda^{4}\right. \\
& \left.+E_{4} \lambda^{3}+E_{5} \lambda^{2}+E_{6} \lambda+E_{7}\right) e^{-\lambda \tau_{3}}=0,
\end{aligned}
$$

where the values of $A_{1-8}, B_{1-7}, C_{1-7}, E_{1-7}$ are showed in the Appendix.

If we assume that $\tau_{1}=\tau_{2}=\tau_{3}=\tau$, we will have

$$
\begin{aligned}
\lambda^{8} & +A_{1} \lambda^{7}+A_{2} \lambda^{6}+A_{3} \lambda^{5}+A_{4} \lambda^{4}+A_{5} \lambda^{3}+A_{6} \lambda^{2} \\
& +A_{7} \lambda+A_{8}+\left(D_{1} \lambda^{6}+D_{2} \lambda^{5}+D_{3} \lambda^{4}+D_{4} \lambda^{3}\right. \\
& \left.+D_{5} \lambda^{2}+D_{6} \lambda+D_{7}\right) e^{-\lambda \tau}=0,
\end{aligned}
$$

where $D_{j}=B_{j}+C_{j}+E_{j}, \quad j=1,2,3,4,5,6,7$.

(1) If $\tau=0$, (7) becomes

$$
\begin{gathered}
\lambda^{8}+G_{1} \lambda^{7}+G_{2} \lambda^{6}+G_{3} \lambda^{5}+G_{4} \lambda^{4}+G_{5} \lambda^{3}+G_{6} \lambda^{2} \\
+G_{7} \lambda+G_{8}=0,
\end{gathered}
$$

where

$$
\begin{aligned}
& G_{1}=A_{1}, \\
& G_{2}=A_{2}+D_{1}, \\
& G_{3}=A_{3}+D_{2}, \\
& G_{4}=A_{4}+D_{3}, \\
& G_{5}=A_{5}+D_{4}, \\
& G_{6}=A_{6}+D_{5}, \\
& G_{7}=A_{7}+D_{6}, \\
& G_{8}=A_{8}+D_{7} .
\end{aligned}
$$

According to the Routh-Hurwitz criterion, all roots of (8) have negative real parts if and only if all the subdeterminants in the diagonal are positive; that is,

$$
\begin{aligned}
& H_{1}=G_{1}>0 \text {, } \\
& H_{2}=\left|\begin{array}{cc}
G_{1} & 1 \\
G_{3} & G_{2}
\end{array}\right|>0 \text {, } \\
& H_{3}=\left|\begin{array}{ccc}
G_{1} & 1 & 0 \\
G_{3} & G_{2} & G_{1} \\
G_{5} & G_{4} & G_{3}
\end{array}\right|>0 \text {, } \\
& H_{4}=\left|\begin{array}{cccc}
G_{1} & 1 & 0 & 0 \\
G_{3} & G_{2} & G_{1} & 1 \\
G_{5} & G_{4} & G_{3} & G_{2} \\
G_{7} & G_{6} & G_{5} & G_{4}
\end{array}\right|>0 \text {, } \\
& H_{5}=\left|\begin{array}{ccccc}
G_{1} & 1 & 0 & 0 & 0 \\
G_{3} & G_{2} & G_{1} & 1 & 0 \\
G_{5} & G_{4} & G_{3} & G_{2} & G_{1} \\
G_{7} & G_{6} & G_{5} & G_{4} & G_{3} \\
0 & G_{8} & G_{7} & G_{6} & G_{5}
\end{array}\right|>0, \\
& H_{6}=\left|\begin{array}{cccccc}
G_{1} & 1 & 0 & 0 & 0 & 0 \\
G_{3} & G_{2} & G_{1} & 1 & 0 & 0 \\
G_{5} & G_{4} & G_{3} & G_{2} & G_{1} & 1 \\
G_{7} & G_{6} & G_{5} & G_{4} & G_{3} & G_{2} \\
0 & G_{8} & G_{7} & G_{6} & G_{5} & G_{4} \\
0 & 0 & 0 & G_{8} & G_{7} & G_{6}
\end{array}\right|>0, \\
& H_{7}=\left|\begin{array}{ccccccc}
G_{1} & 1 & 0 & 0 & 0 & 0 & 0 \\
G_{3} & G_{2} & G_{1} & 1 & 0 & 0 & 0 \\
G_{5} & G_{4} & G_{3} & G_{2} & G_{1} & 1 & 0 \\
G_{7} & G_{6} & G_{5} & G_{4} & G_{3} & G_{2} & G_{1} \\
0 & G_{8} & G_{7} & G_{6} & G_{5} & G_{4} & G_{3} \\
0 & 0 & 0 & G_{8} & G_{7} & G_{6} & G_{5} \\
0 & 0 & 0 & 0 & 0 & G_{8} & G_{7}
\end{array}\right|>0, \\
& H_{8}=G_{8} H_{7}>0 \text {. }
\end{aligned}
$$

(2) If $\tau>0$, considering the transcendental equation (7), clearly $i \omega(\omega>0)$ is a root of (7) if and only if

$$
\begin{aligned}
- & \omega^{8}+A_{2} \omega^{6}-A_{4} \omega^{4}+A_{6} \omega^{2}-A_{8} \\
+ & i\left(A_{1} \omega^{7}-A_{3} \omega^{5}+A_{5} \omega^{3}-A_{7} \omega\right) \\
= & -D_{1} \omega^{6}+D_{3} \omega^{4}-D_{5} \omega^{2}+D_{7} \\
& +i\left(D_{2} \omega^{5}-D_{4} \omega^{3}+D_{6} \omega\right)(\cos (\omega \tau)-i \sin (\omega \tau)) .
\end{aligned}
$$


Separating the real and imaginary parts of (11), we have

$$
\begin{aligned}
&-\omega^{8}+ A_{2} \omega^{6}-A_{4} \omega^{4}+A_{6} \omega^{2}-A_{8} \\
&=\left(-D_{1} \omega^{6}+D_{3} \omega^{4}-D_{5} \omega^{2}+D_{7}\right) \cos (\omega \tau) \\
&+\left(D_{2} \omega^{5}-D_{4} \omega^{3}+D_{6} \omega\right) \sin (\omega \tau), \\
& A_{1} \omega^{7}-A_{3} \omega^{5}+A_{5} \omega^{3}-A_{7} \omega \\
&=\left(D_{1} \omega^{6}-D_{3} \omega^{4}+D_{5} \omega^{2}-D_{7}\right) \sin (\omega \tau) \\
&+\left(D_{2} \omega^{5}-D_{4} \omega^{3}+D_{6} \omega\right) \cos (\omega \tau) .
\end{aligned}
$$

Adding up the squares of both equations of (12), we have

$$
\begin{aligned}
\omega^{16}+ & F_{1} \omega^{14}+F_{2} \omega^{12}+F_{3} \omega^{10}+F_{4} \omega^{8}+F_{5} \omega^{6}+F_{6} \omega^{4} \\
& +F_{7} \omega^{2}+F_{8}=0,
\end{aligned}
$$

where

$$
\begin{aligned}
F_{1}= & -2 A_{2}+A_{1}^{2}, \\
F_{2}= & -D_{1}^{2}-2 A_{1} A_{3}+A_{2}^{2}+2 A_{4}, \\
F_{3}= & 2 A_{1} A_{5}-2 A_{2} A_{4}-D_{2}^{2}+A_{3}^{2}-2 A_{6}+2 D_{1} D_{3}, \\
F_{4}= & -2 A_{3} A_{5}-2 A_{1} A_{7}-D_{3}^{2}+2 D_{2} D_{4}+2 A_{2} A_{6} \\
& +2 A_{8}+A_{4}^{2}-2 D_{1} D_{5}, \\
F_{5}= & -D_{4}^{2}-2 A_{4} A_{6}-2 A_{2} A_{8}-2 D_{2} D_{6}+2 D_{3} D_{5} \\
& +2 D_{1} D_{7}+2 A_{3} A_{7}+A_{5}^{2}, \\
F_{6}= & -2 D_{3} D_{7}+2 D_{4} D_{6}+2 A_{4} A_{8}-2 A_{5} A_{7}+A_{6}^{2} \\
& -D_{5}^{2}, \\
F_{7}= & -D_{6}^{2}-2 A_{6} A_{8}+2 D_{5} D_{7}+A_{7}^{2}, \\
F_{8}= & -D_{7}^{2}+A_{8}^{2} .
\end{aligned}
$$

Let $z=\omega^{2}$; (13) becomes

$$
\begin{aligned}
z^{8}+ & F_{1} z^{7}+F_{2} z^{6}+F_{3} z^{5}+F_{4} z^{4}+F_{5} z^{3}+F_{6} z^{2}+F_{7} z \\
+ & F_{8}=0 .
\end{aligned}
$$

Denote

$$
\begin{aligned}
h(z)= & z^{8}+F_{1} z^{7}+F_{2} z^{6}+F_{3} z^{5}+F_{4} z^{4}+F_{5} z^{3}+F_{6} z^{2} \\
& +F_{7} z+F_{8} .
\end{aligned}
$$

Lemma 1. If $F_{8}<0$, (15) has at least one positive root.

Proof. Clearly, $h(0)=F_{8}<0$, and $\lim _{z \rightarrow \infty} h(z)=\infty$. Hence, there exists $z_{0} \in(0, \infty)$, so that $h\left(z_{0}\right)=0$. This completes the proof.

Lemma 2. If $F_{8}>0$, the sufficient condition for (15) has positive roots being $z^{\prime}(7)>0$ and $h\left(z^{\prime}(7)\right)<0$.
Proof. From (16), we have $h^{\prime}(z)=8 z^{7}+7 F_{1} z^{6}+6 F_{2} z^{5}+5 F_{4} z+$ $4 F_{4} z^{3}+3 F_{5} z^{2}+2 F_{6} z+F_{7}$; suppose the equation $h^{\prime}(z)=0$ has seven real roots and satisfies $z^{\prime}(1)<z^{\prime}(2)<z^{\prime}(3)<z^{\prime}(4)<$ $z^{\prime}(5)<z^{\prime}(6)<z^{\prime}(7)$, and $z^{\prime}(7)$ is the local minimum value, if $z^{\prime}(7)>0$ and $h\left(z^{\prime}(7)\right)<0$; there exists $z \in\left(z^{\prime}(7), \infty\right)$, so that $h(z)=0$; this completes the proof.

Lemma 3. If $F_{8}>0$, according to the Routh-Hurwitz criterion, all the roots of (15) have negative real parts if and only if the subdeterminants in the diagonal are positive, that is, $\Delta j>0$, $j=1,2, \ldots, 8$.

Suppose that (15) has positive roots; without loss of generality, we assume that it has eight positive roots, denoted by $z_{1}, z_{2}, z_{3}, z_{4}, z_{5}, z_{6}, z_{7}, z_{8}$, respectively. Hence, (13) has eight positive roots, say $\omega_{1}=\sqrt{z_{1}}, \omega_{2}=\sqrt{z_{2}}, \omega_{3}=\sqrt{z_{3}}, \omega_{4}=$ $\sqrt{z_{4}}, \omega_{5}=\sqrt{z_{5}}, \omega_{6}=\sqrt{z_{6}}, \omega_{7}=\sqrt{z_{7}}, \omega_{8}=\sqrt{z_{8}}$.

From (12), we can get

$$
\begin{aligned}
\tau_{k}^{j}=\arccos \left(\frac{R_{1} R_{2}+R_{3} R_{4}}{R_{2}^{2}+R_{4}^{2}}\right), \\
\quad k=1,2,3, \ldots, 8, j=0,1,2,3, \ldots,
\end{aligned}
$$

where

$$
\begin{aligned}
& R_{1}=-\omega_{k}^{8}+A_{2} \omega_{k}^{6}-A_{4} \omega_{k}^{4}+A_{6} \omega_{k}^{2}-A_{8}, \\
& R_{2}=-D_{1} \omega_{k}^{6}+D_{3} \omega_{k}^{4}-D_{5} \omega_{k}^{2}+D_{7}, \\
& R_{3}=A_{1} \omega_{k}^{7}-A_{3} \omega_{k}^{5}+A_{5} \omega_{k}^{3}-A_{7} \omega_{k}, \\
& R_{4}=D_{2} \omega_{k}^{5}-D_{4} \omega_{k}^{3}+D_{6} \omega_{k} .
\end{aligned}
$$

Define

$$
\tau_{0}=\tau_{k_{0}}^{j_{0}}=\min _{\substack{1 \leq k \leq 8 \\ j \geq 0}}\left\{\tau_{k}^{j}\right\} .
$$

Let $\lambda(\tau)=\eta(\tau)+i \omega(\tau)$ be the root of (7) satisfying $\eta\left(\tau_{0}\right)=$ $0, \omega\left(\tau_{0}\right)=\omega_{0}$.

Lemma 4. Consider the exponential polynomial $P\left(\lambda, e^{-\lambda \tau_{1}}\right.$, $\left.\ldots, e^{-\lambda \tau_{m}}\right)=p_{1}(\lambda)+p_{2}(\lambda) e^{-\lambda \tau_{1}}+\cdots+p_{m}(\lambda) e^{-\lambda \tau_{m}}$, where $\tau_{i}>0(i=1,2, \ldots, m)$ and $p_{i}(\lambda)$ is polynomial about $\lambda$. As $\left(\tau_{1}, \tau_{2}, \ldots, \tau_{m}\right)$ vary, the sum of the orders of the zeros of $P\left(\lambda, e^{-\lambda \tau_{1}}, \ldots, e^{-\lambda \tau_{m}}\right)$ on the open right half plane can change only if a zero appears on or cross the imaginary axis.

Then, we have the following theoretical results.

Theorem 5. Suppose that conditions (10) are satisfied.

(i) If $\Delta j>0(j=1,2, \ldots, 8)$, then all roots of (7) have negative real parts for all $\tau \geq 0$; thus the steady state $\left(x_{10}, x_{20}, x_{30}, x_{40}, x_{50}, x_{60}, x_{70}, x_{80}\right)$ of system (2) is absolutely stable.

(ii) If $F_{8}<0$ or $F_{8} \geq 0, z^{\prime}(7)>0$, and $h\left(z^{\prime}(7)\right)<0$, then all the roots of (7) have negative real parts when $\tau \in\left[0, \tau_{0}\right)$; thus the steady state of system (2) is asymptotically stable. 
(iii) If the condition of (ii) is satisfied, $\tau=\tau_{0}$, and $h^{\prime}\left(z_{0}\right) \neq$ $0, z_{0}=\omega_{0}^{2}$, then $\pm i \omega_{0}$ is a pair of simple purely imaginary roots of (15) and all other roots have negative real parts. Moreover, $\left.\left(d \operatorname{Re} \lambda\left(\tau_{0}\right) / d \tau\right)\right|_{\tau=\tau_{0}}>0$. Thus, system (2) exhibits the Hopf bifurcation at $\left(x_{10}, x_{20}, x_{30}\right.$, $\left.x_{40}, x_{50}, x_{60}, x_{70}, x_{80}\right)$.

\section{Numerical Analysis}

In this section, we demonstrate the above theoretical results by numerical method. When we take $S=5, k_{21}=1.4$, and the other parameters are shown in Table 1, system (1) becomes

$$
\begin{array}{rl}
\frac{d x_{1}}{d t}= & \frac{0.4 x_{3} x_{1}}{\left(0.15+x_{3}\right)\left(0.15+x_{1}\right)}+\frac{0.003 x_{3}}{0.15+x_{3}} \\
& +\frac{18 x_{4} x_{8}}{0.92+x_{8}}+\frac{18 x_{5} x_{8}}{0.92+x_{8}}-0.25 x_{1} \\
& -180 x_{6} x_{1}, \\
\frac{d x_{2}}{d t}= & \frac{1.4 x_{1}}{0.15+x_{1}}-0.02 x_{2}-\frac{0.6 x_{2} x_{3}}{0.15+x_{3}}-\frac{x_{2} x_{4}}{0.92+x_{4}} \\
& -\frac{0.7 x_{2} x_{5}}{0.92+x_{5}}, \\
\frac{d x_{3}}{d t}= & 0.9090909090-0.7 x_{3}-\frac{0.6 x_{2}\left(t-\tau_{1}\right) x_{3}}{0.15+x_{3}}, \\
\frac{d x_{4}}{d t}= & 0.4090909090+\frac{0.03 x_{3}}{0.15+x_{3}}-1.5 x_{4} \\
& -\frac{x_{2}\left(t-\tau_{2}\right) x_{4}}{0.92+x_{4}}, \\
\frac{d x_{5}}{d t}= & \frac{0.35 x_{1}}{0.15+x_{1}}-1.5 x_{5}-\frac{0.7 x_{2}\left(t-\tau_{3}\right) x_{5}}{0.92+x_{5}}, \\
\frac{d x_{6}}{d t}= & 0.18+\frac{3.6 x_{7}}{0.01+x_{7}}-180 x_{6} x_{1}-\frac{18 x_{4} x_{6}}{0.92+x_{6}} \\
\frac{d x_{7}}{d t}= & \frac{18 x_{4} x_{6}}{0.92+x_{6}}+\frac{18 x_{5} x_{6}}{0.92+x_{6}}+\frac{18 x_{4} x_{8}}{0.92+x_{8}} \\
& +\frac{18 x_{5} x_{8}}{0.92+x_{8}}-\frac{3.6 x_{7}}{0.01+x_{7} x_{6}}-0.06 x_{7}, \\
0.92+x_{6} & 180 x_{6} x_{1}-\frac{18 x_{4} x_{8}}{0.92+x_{8}}-\frac{18 x_{5} x_{8}}{0.92+x_{8}}-0.03 x_{8} .
\end{array}
$$

The system has a positive equilibrium point $Z=(0.4102$, $1.6027,0.3430,0.1429,0.0985,0.0478,1.2301,3.4433)$. Using Theorem 5, there is a critical value of the time delay $\tau_{0}=$ 0.122632125 . The equilibrium point is stable when $\tau<\tau_{0}$ (see Figures 2 and 3); the equilibrium point becomes unstable and a Hopf bifurcation occurs when $\tau$ passes through the critical value $\tau_{0}$ (see Figure 4 ). The bifurcation diagrams of system

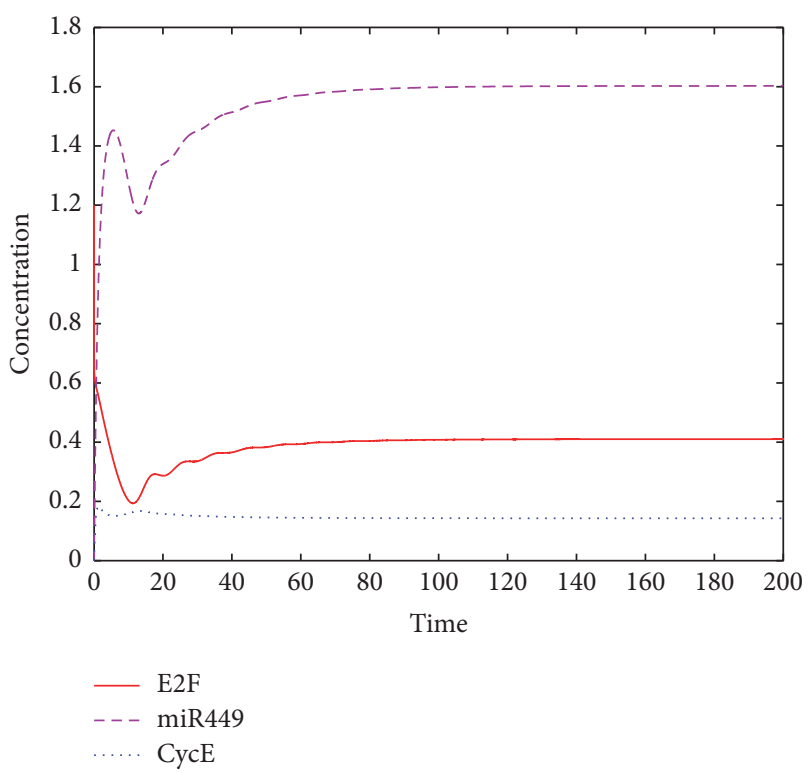

FIgURE 2: An asymptotically stable equilibrium for $\tau=\tau_{1}=\tau_{2}=$ $\tau_{3}=0$. Assume initial conditions are $x_{1}=1.2, x_{2}=0, x_{3}=0, x_{4}=$ $0, x_{5}=0, x_{6}=0.55, x_{7}=0, x_{8}=0$, and $S=5, k_{21}=1.4$.

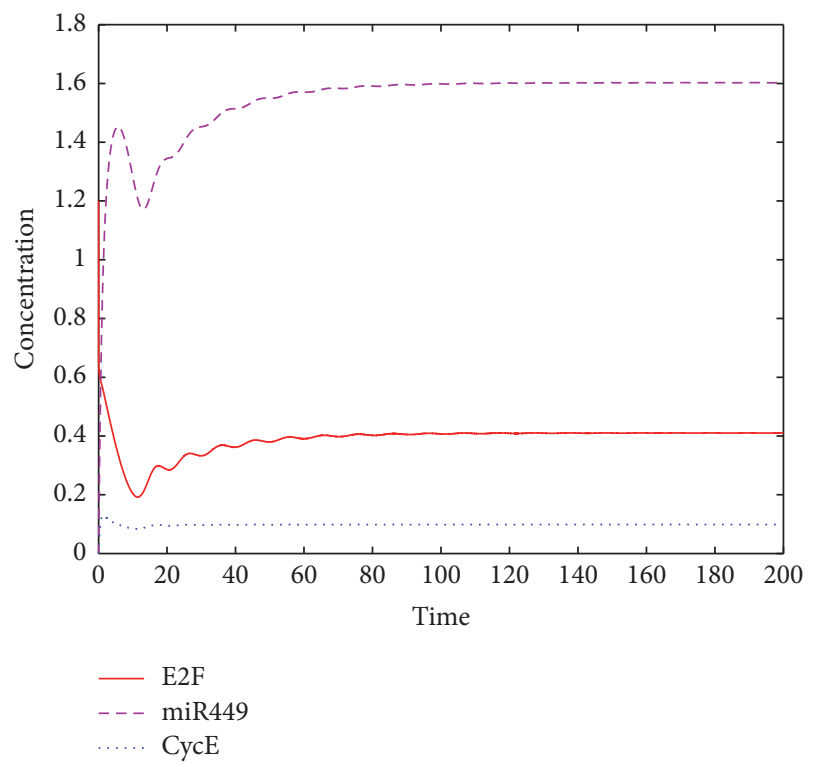

FIGURE 3: An asymptotically stable equilibrium for $\tau=\tau_{1}=\tau_{2}=$ $\tau_{3}<\tau_{0}$. Assume initial conditions are $x_{1}=1.2, x_{2}=0, x_{3}=$ $0, x_{4}=0, x_{5}=0, x_{6}=0.55, x_{7}=0, x_{8}=0$, and $S=5, k_{21}=1.4$.

(20) are shown in Figure 5, where the control parameter is the time delay $\tau$.

When we take $S=3, k_{21}=2.5$, and the other parameters are shown in Table 1, system (1) becomes

$$
\begin{aligned}
\frac{d x_{1}}{d t}= & \frac{0.4 x_{3} x_{1}}{\left(0.15+x_{3}\right)\left(0.15+x_{1}\right)}+\frac{0.003 x_{3}}{0.15+x_{3}} \\
& +\frac{18 x_{4} x_{8}}{0.92+x_{8}}+\frac{18 x_{5} x_{8}}{0.92+x_{8}}-0.25 x_{1}
\end{aligned}
$$




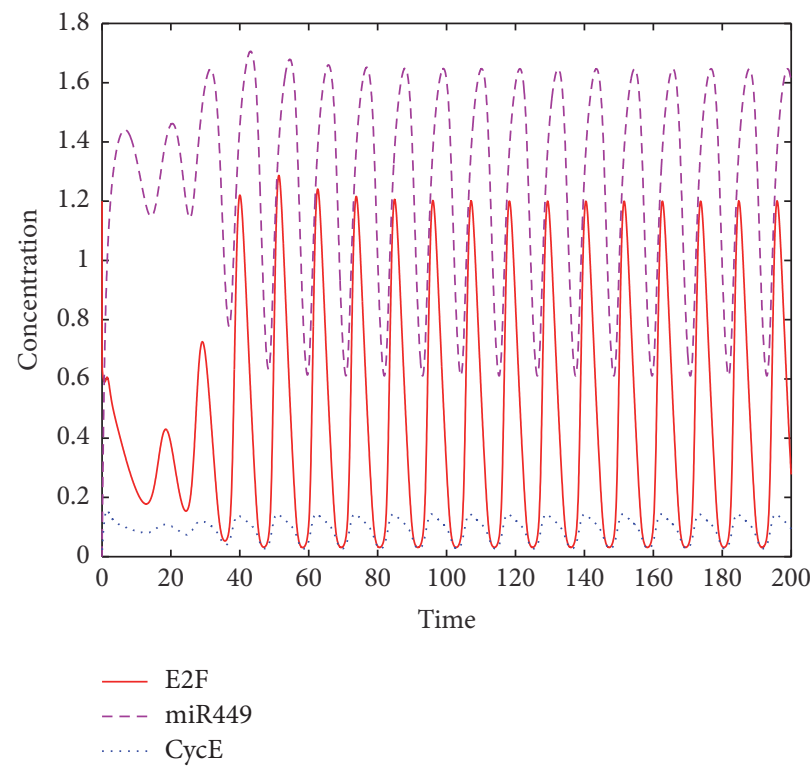

FIGURE 4: A periodic solution bifurcated from equilibrium for $\tau=\tau_{1}=\tau_{2}=\tau_{3}>\tau_{0}$. Assume initial conditions are $x_{1}=1.2, x_{2}=0, x_{3}=$ $0, x_{4}=0, x_{5}=0, x_{6}=0.55, x_{7}=0, x_{8}=0$, and $S=5, k_{21}=1.4$.
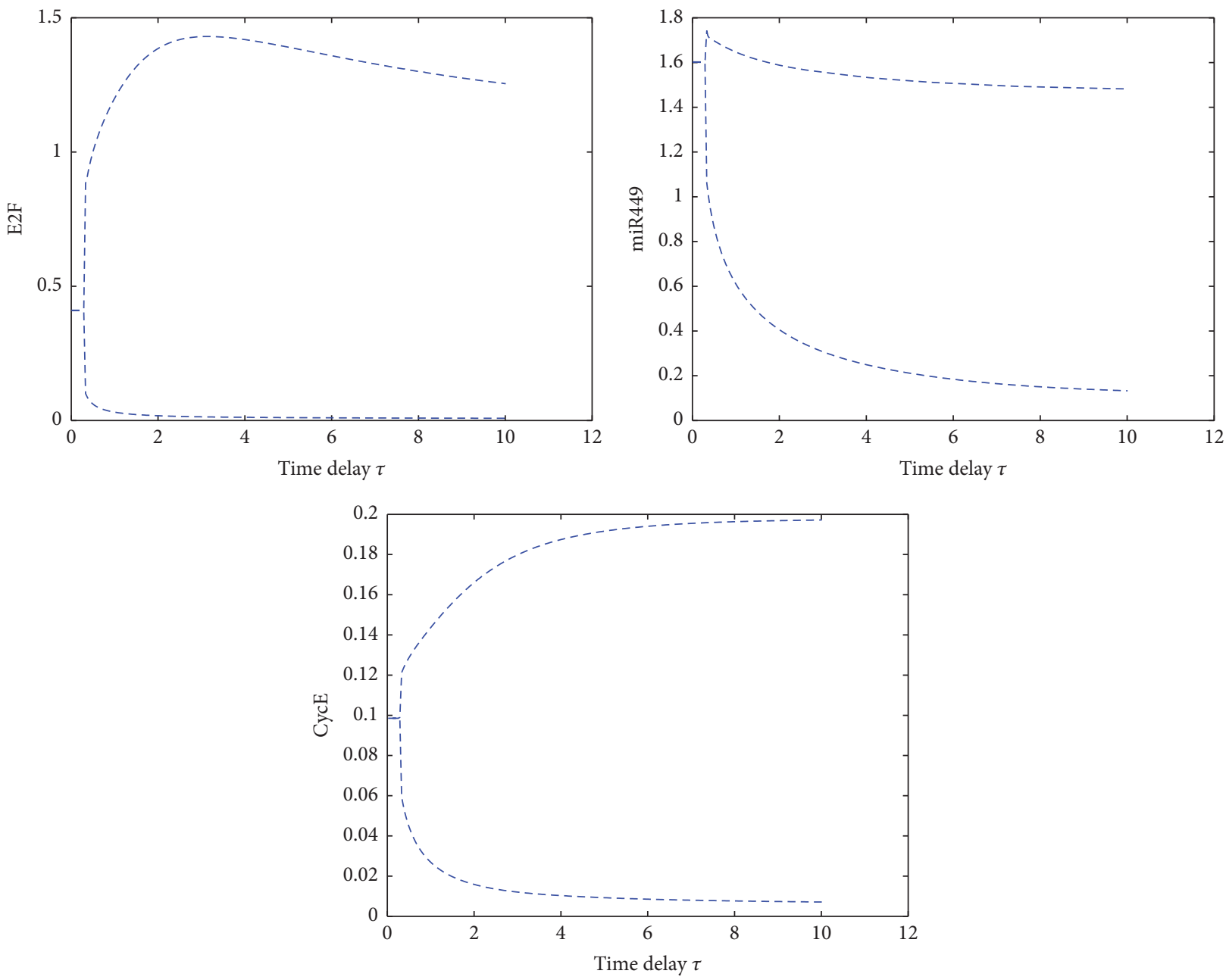

FIGURE 5: Bifurcation induced by time delay with $S=5, k_{21}=1.4$. Assume initial conditions are $x_{1}=1.2, x_{2}=0, x_{3}=0, x_{4}=0, x_{5}=$ $0, x_{6}=0.55, x_{7}=0, x_{8}=0$, and $S=5, k_{21}=1.4$. 


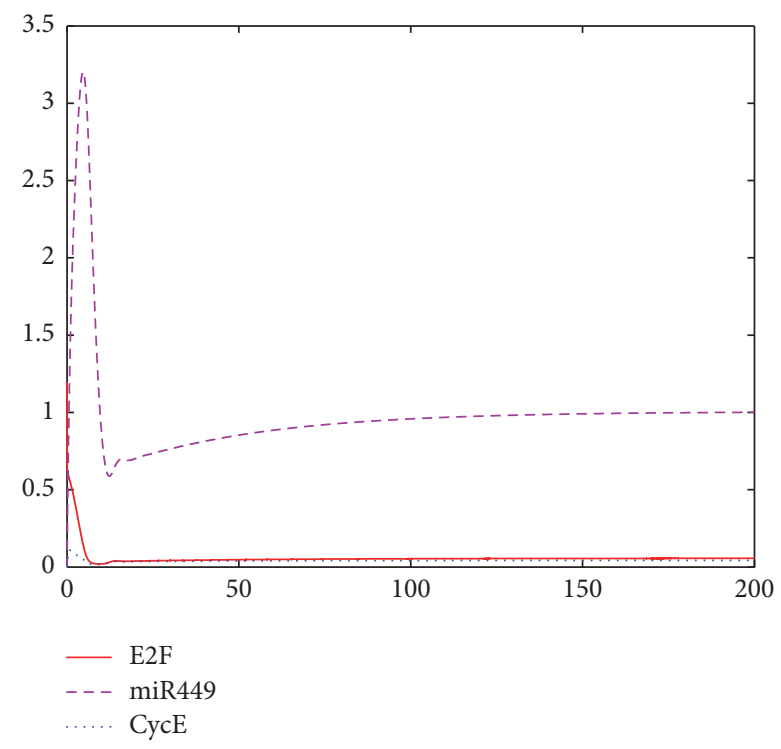

Figure 6: An asymptotically stable equilibrium for $\tau=\tau_{1}=\tau_{2}=$ $\tau_{3}=0$. Assume initial conditions are $x_{1}=1.2, x_{2}=0, x_{3}=0, x_{4}=$ $0, x_{5}=0, x_{6}=0.55, x_{7}=0, x_{8}=0$, and $S=3, k_{21}=2.5$.

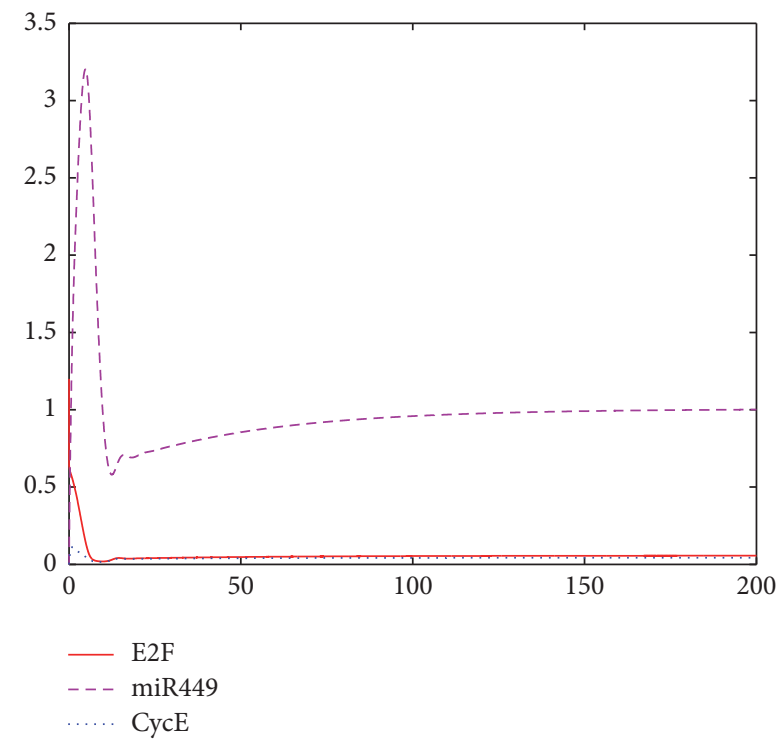

Figure 7: An asymptotically stable equilibrium for $\tau=\tau_{1}=\tau_{2}=$ $\tau_{3}<\tau_{0}$. Assume initial conditions are $x_{1}=1.2, x_{2}=0, x_{3}=$ $0, x_{4}=0, x_{5}=0, x_{6}=0.55, x_{7}=0, x_{8}=0$, and $S=3, k_{21}=2.5$.

$$
\begin{aligned}
& -180 x_{6} x_{1}, \\
\frac{d x_{2}}{d t}= & \frac{1.4 x_{1}}{0.15+x_{1}}-0.02 x_{2}-\frac{0.6 x_{2} x_{3}}{0.15+x_{3}}-\frac{x_{2} x_{4}}{0.92+x_{4}} \\
& -\frac{0.7 x_{2} x_{5}}{0.92+x_{5}}, \\
\frac{d x_{3}}{d t}= & 0.9090909090-0.7 x_{3}-\frac{0.6 x_{2}\left(t-\tau_{1}\right) x_{3}}{0.15+x_{3}},
\end{aligned}
$$

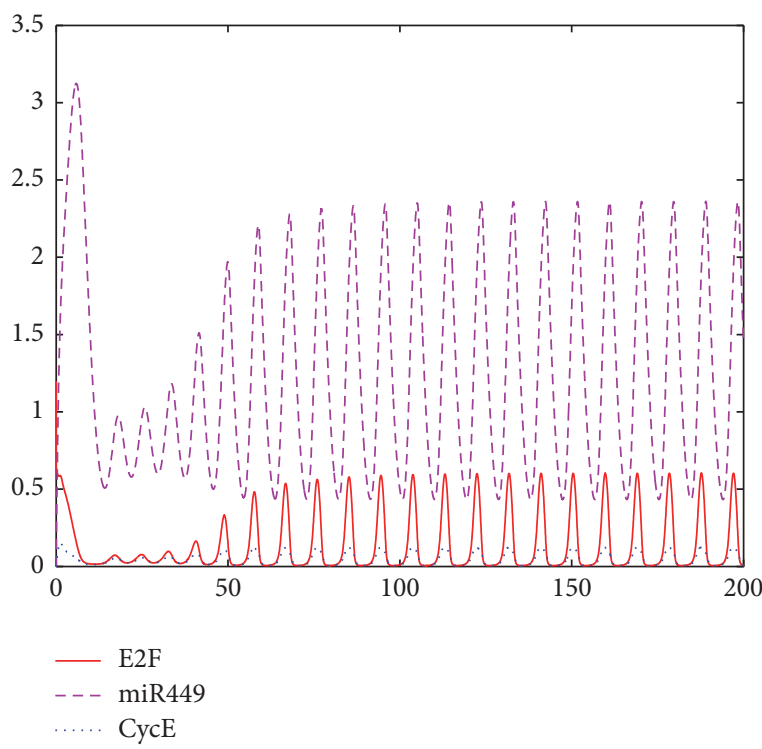

Figure 8: A periodic solution bifurcated from equilibrium for $\tau=$ $\tau_{1}=\tau_{2}=\tau_{3}>\tau_{0}$. Assume initial conditions are $x_{1}=1.2, x_{2}=$ $0, x_{3}=0, x_{4}=0, x_{5}=0, x_{6}=0.55, x_{7}=0, x_{8}=0$, and $S=3, k_{21}=2.5$.

$$
\begin{aligned}
\frac{d x_{4}}{d t}= & 0.4090909090+\frac{0.03 x_{3}}{0.15+x_{3}}-1.5 x_{4} \\
& -\frac{x_{2}\left(t-\tau_{2}\right) x_{4}}{0.92+x_{4}}, \\
\frac{d x_{5}}{d t}= & \frac{0.35 x_{1}}{0.15+x_{1}}-1.5 x_{5}-\frac{0.7 x_{2}\left(t-\tau_{3}\right) x_{5}}{0.92+x_{5}}, \\
\frac{d x_{6}}{d t}= & 0.18+\frac{3.6 x_{7}}{0.01+x_{7}}-180 x_{6} x_{1}-\frac{18 x_{4} x_{6}}{0.92+x_{6}} \\
& -\frac{18 x_{5} x_{6}}{0.92+x_{6}}-0.06 x_{6}, \\
\frac{d x_{7}}{d t}= & \frac{18 x_{4} x_{6}}{0.92+x_{6}}+\frac{18 x_{5} x_{6}}{0.92+x_{6}}+\frac{18 x_{4} x_{8}}{0.92+x_{8}} \\
& +\frac{18 x_{5} x_{8}}{0.92+x_{8}}-\frac{3.6 x_{7}}{0.01+x_{7}}-0.06 x_{7}, \\
\frac{d x_{8}}{d t}= & 180 x_{6} x_{1}-\frac{18 x_{4} x_{8}}{0.92+x_{8}}-\frac{18 x_{5} x_{8}}{0.92+x_{8}}-0.03 x_{8} .
\end{aligned}
$$

The system has a positive equilibrium point $Z^{\prime}=(0.0561$, $1.0057,0.5478,0.1689,0.0427,0.2816,1.4875,2.4618)$. Using Theorem 5 , there is a critical value of the time delay $\tau_{0}^{\prime}=$ 0.1334173317 . The equilibrium point is stable when $\tau<\tau_{0}^{\prime}$ (see Figures 6 and 7); the equilibrium point becomes unstable and a Hopf bifurcation occurs when $\tau$ passes through the critical value $\tau_{0}^{\prime}$ (see Figure 8 ). The bifurcation diagrams of system (21) are shown in Figure 9, where the control parameter is the time delay $\tau$. 

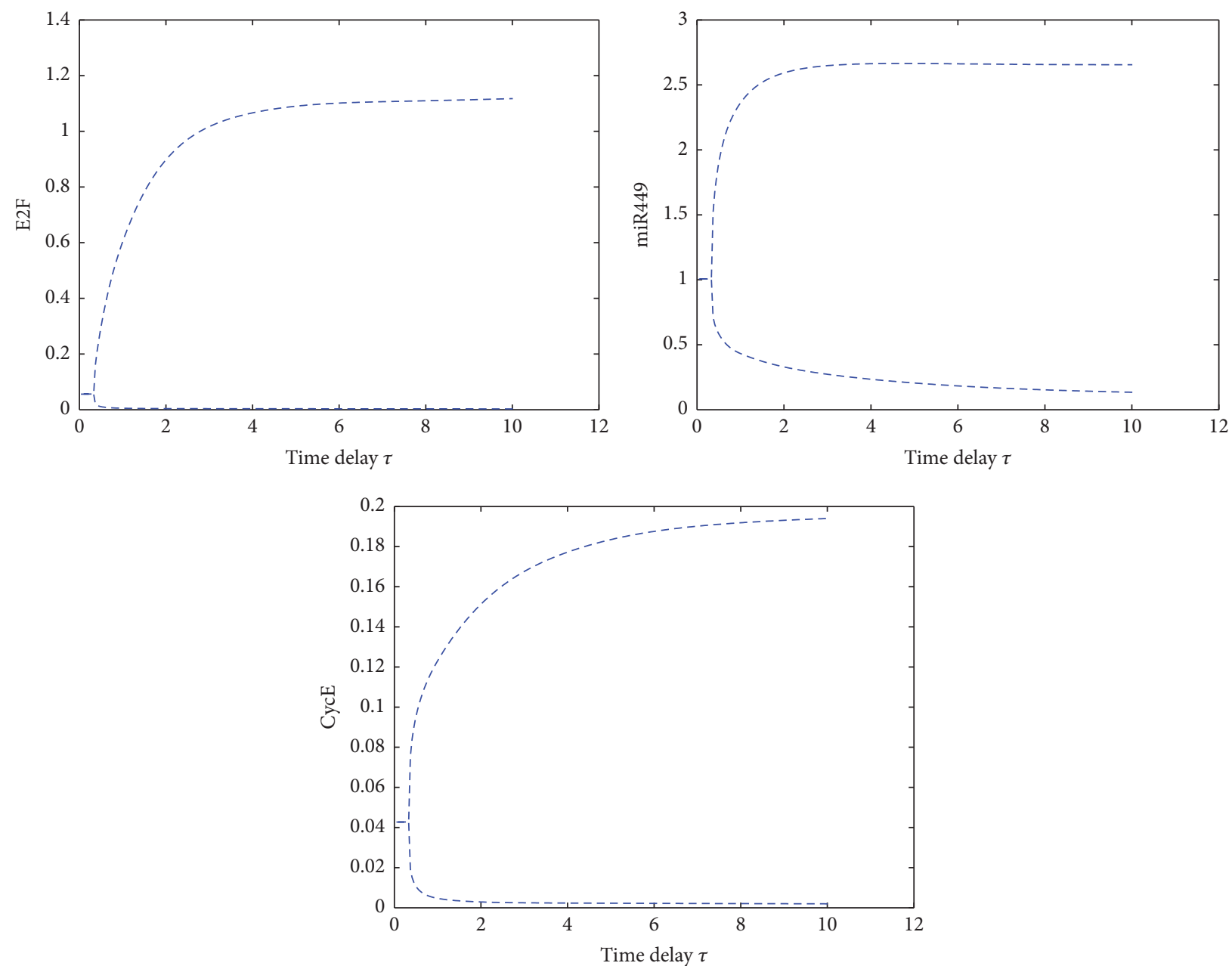

Figure 9: Bifurcation induced by time delay with $S=3, k_{21}=2.5$. Assume initial conditions are $x_{1}=1.2, x_{2}=0, x_{3}=0, x_{4}=0, x_{5}=$ $0, x_{6}=0.55, x_{7}=0, x_{8}=0$, and $S=3, k_{21}=2.5$.

\section{Discussion}

In this paper, we studied the dynamical behaviors of the Rb-E2F pathway including negative feedback loops involving miR449 by using Hopf bifurcation theory. On one hand, we gave the detailed theoretical analysis of system (1) in which there are some dynamical behaviors, and it is shown that, under certain conditions, the equilibrium point is asymptotically stable for all the delay $\tau \geq 0$; however, if these conditions are not met, there is a critical delay $\tau_{0}$; when $\tau<\tau_{0}$, the equilibrium point is asymptotically stable; when $\tau>\tau_{0}$, periodic oscillations occur. Thus, Hopf bifurcation appears at the steady state when the delay passes through the critical value $\tau_{0}$. On the other hand, through numerical simulations, we calculated the critical value of $\tau_{0}$, giving the time courses of E2F, CycE, and miR449 both $\tau<\tau_{0}$ and $\tau>\tau_{0}$, drawing the bifurcation diagrams of E2F, CycE, and miR449, respectively, and testifying the correctness of the theory.

\section{Appendix}

$$
\begin{aligned}
& A_{1}=-a_{66}-a_{22}-a_{11}-a_{55}-a_{33}-a_{44}-a_{88}-a_{77} \\
& A_{2}=a_{33} a_{44}-a_{61} a_{16}-a_{81} a_{18}+a_{11} a_{22}+a_{11} a_{66}+a_{44} a_{66}+a_{66} a_{88}+a_{33} a_{88}+a_{22} a_{77}-a_{76} a_{67}+a_{11} a_{88}+a_{33} a_{66}+a_{55} a_{88}+a_{55} a_{77} \\
& +a_{22} a_{66}+a_{44} a_{88}+a_{33} a_{55}-a_{51} a_{15}+a_{55} a_{11}+a_{66} a_{77}+a_{55} a_{22}+a_{44} a_{55}+a_{33} a_{22}+a_{44} a_{77}+a_{33} a_{11}+a_{77} a_{88}+a_{44} a_{22}+a_{22} a_{88} \\
& +a_{55} a_{66}+a_{11} a_{77}+a_{44} a_{11}+a_{33} a_{77} \\
& A_{3}=-a_{33} a_{44} a_{55}-a_{33} a_{66} a_{88}-a_{33} a_{66} a_{77}-a_{33} a_{55} a_{22}-a_{44} a_{11} a_{88}-a_{44} a_{11} a_{22}-a_{44} a_{66} a_{77}+a_{55} a_{61} a_{16}+a_{33} a_{61} a_{16}-a_{44} a_{55} a_{77} \\
& +a_{44} a_{81} a_{18}-a_{44} a_{22} a_{66}-a_{33} a_{55} a_{11}-a_{33} a_{77} a_{88}-a_{44} a_{55} a_{22}-a_{44} a_{55} a_{88}-a_{33} a_{22} a_{77}+a_{44} a_{61} a_{16}-a_{33} a_{44} a_{77}-a_{11} a_{22} a_{66}
\end{aligned}
$$




$$
\begin{aligned}
& -a_{55} a_{66} a_{88}-a_{33} a_{55} a_{77}-a_{33} a_{22} a_{66}-a_{44} a_{22} a_{77}-a_{44} a_{55} a_{11}-a_{44} a_{11} a_{77}-a_{55} a_{66} a_{77}-a_{44} a_{22} a_{88}+a_{44} a_{76} a_{67}-a_{33} a_{44} a_{88} \\
& -a_{33} a_{11} a_{77}-a_{44} a_{66} a_{88}-a_{55} a_{11} a_{77}-a_{33} a_{55} a_{88}-a_{33} a_{22} a_{88}-a_{55} a_{11} a_{22}+a_{33} a_{81} a_{18}-a_{33} a_{11} a_{66}-a_{33} a_{11} a_{22}-a_{44} a_{11} a_{66} \\
& +a_{33} a_{76} a_{67}-a_{33} a_{11} a_{88}-a_{33} a_{44} a_{22}-a_{44} a_{77} a_{88}+a_{55} a_{81} a_{18}-a_{61} a_{86} a_{18}+a_{61} a_{22} a_{16}+a_{81} a_{22} a_{18}+a_{81} a_{18} a_{66}-a_{55} a_{22} a_{66} \\
& -a_{33} a_{44} a_{66}-a_{33} a_{44} a_{11}-a_{55} a_{77} a_{88}-a_{44} a_{55} a_{66}-a_{55} a_{11} a_{66}-a_{55} a_{22} a_{88}+a_{55} a_{76} a_{67}-a_{55} a_{11} a_{88}+a_{81} a_{18} a_{77}-a_{55} a_{22} a_{77} \\
& +a_{51} a_{15} a_{66}+a_{51} a_{33} a_{15}+a_{51} a_{15} a_{88}-a_{51} a_{65} a_{16}+a_{51} a_{15} a_{77}-a_{11} a_{77} a_{88}-a_{51} a_{85} a_{18}+a_{22} a_{76} a_{67}+a_{11} a_{76} a_{67}-a_{11} a_{66} a_{88} \\
& -a_{11} a_{66} a_{77}-a_{66} a_{77} a_{88}+a_{61} a_{16} a_{88}-a_{11} a_{22} a_{88}+a_{61} a_{16} a_{77}-a_{22} a_{66} a_{88}-a_{22} a_{77} a_{88}+a_{76} a_{67} a_{88}-a_{86} a_{67} a_{78}-a_{11} a_{22} a_{77} \\
& +a_{51} a_{22} a_{15}+a_{51} a_{44} a_{15}-a_{22} a_{66} a_{77}-a_{33} a_{55} a_{66} \text {, } \\
& A_{4}=a_{44} a_{11} a_{22} a_{77}+a_{44} a_{61} a_{86} a_{18}-a_{44} a_{61} a_{22} a_{16}-a_{44} a_{81} a_{18} a_{77}+a_{44} a_{55} a_{11} a_{66}-a_{44} a_{81} a_{18} a_{66}-a_{44} a_{81} a_{22} a_{18}+a_{44} a_{55} a_{22} a_{77} \\
& +a_{44} a_{55} a_{77} a_{88}+a_{44} a_{55} a_{22} a_{88}-a_{44} a_{55} a_{76} a_{67}+a_{44} a_{55} a_{66} a_{88}-a_{44} a_{55} a_{61} a_{16}+a_{44} a_{55} a_{11} a_{77}+a_{44} a_{55} a_{11} a_{22}-a_{44} a_{55} a_{81} a_{18} \\
& -a_{33} a_{22} a_{76} a_{67}+a_{33} a_{11} a_{77} a_{88}+a_{33} a_{11} a_{66} a_{88}+a_{33} a_{11} a_{66} a_{77}-a_{33} a_{11} a_{76} a_{67}+a_{33} a_{11} a_{22} a_{88}+a_{33} a_{11} a_{22} a_{66}-a_{33} a_{61} a_{16} a_{88} \\
& -a_{33} a_{61} a_{16} a_{77}+a_{33} a_{22} a_{66} a_{88}+a_{33} a_{66} a_{77} a_{88}+a_{33} a_{22} a_{66} a_{77}+a_{33} a_{86} a_{67} a_{78}+a_{33} a_{61} a_{86} a_{18}-a_{33} a_{76} a_{67} a_{88}+a_{33} a_{22} a_{77} a_{88} \\
& +a_{33} a_{11} a_{22} a_{77}+a_{51} a_{65} a_{16} a_{88}-a_{51} a_{15} a_{66} a_{77}+a_{51} a_{85} a_{18} a_{66}+a_{51} a_{85} a_{18} a_{77}-a_{51} a_{75} a_{16} a_{67}+a_{44} a_{55} a_{11} a_{88}+a_{44} a_{55} a_{22} a_{66} \\
& +a_{44} a_{55} a_{66} a_{77}-a_{51} a_{33} a_{15} a_{88}-a_{51} a_{15} a_{66} a_{88}-a_{51} a_{65} a_{86} a_{18}+a_{51} a_{22} a_{85} a_{18}-a_{51} a_{22} a_{15} a_{66}-a_{51} a_{22} a_{15} a_{88}-a_{51} a_{22} a_{15} a_{77} \\
& +a_{51} a_{22} a_{65} a_{16}+a_{51} a_{44} a_{85} a_{18}-a_{51} a_{44} a_{15} a_{66}-a_{51} a_{44} a_{15} a_{88}-a_{51} a_{44} a_{15} a_{77}+a_{51} a_{44} a_{65} a_{16}-a_{51} a_{44} a_{22} a_{15}+a_{51} a_{33} a_{85} a_{18} \\
& -a_{51} a_{33} a_{15} a_{66}-a_{51} a_{33} a_{15} a_{77}+a_{51} a_{33} a_{65} a_{16}-a_{51} a_{33} a_{22} a_{15}-a_{51} a_{33} a_{44} a_{15}-a_{81} a_{22} a_{18} a_{77}-a_{11} a_{76} a_{67} a_{88}+a_{11} a_{86} a_{67} a_{78} \\
& +a_{11} a_{22} a_{77} a_{88}+a_{11} a_{22} a_{66} a_{88}-a_{81} a_{16} a_{67} a_{78}-a_{81} a_{18} a_{66} a_{77}+a_{81} a_{76} a_{18} a_{67}-a_{61} a_{16} a_{77} a_{88}+a_{61} a_{86} a_{18} a_{77}-a_{61} a_{22} a_{16} a_{88} \\
& +a_{22} a_{66} a_{77} a_{88}-a_{22} a_{76} a_{67} a_{88}+a_{22} a_{86} a_{67} a_{78}+a_{11} a_{66} a_{77} a_{88}-a_{55} a_{22} a_{76} a_{67}+a_{55} a_{11} a_{77} a_{88}+a_{55} a_{11} a_{66} a_{88}+a_{55} a_{11} a_{66} a_{77} \\
& -a_{55} a_{11} a_{76} a_{67}+a_{55} a_{11} a_{22} a_{88}+a_{55} a_{11} a_{22} a_{66}-a_{55} a_{61} a_{16} a_{88}-a_{55} a_{61} a_{16} a_{77}+a_{55} a_{66} a_{77} a_{88}-a_{55} a_{76} a_{67} a_{88}+a_{55} a_{86} a_{67} a_{78} \\
& +a_{55} a_{22} a_{77} a_{88}+a_{55} a_{22} a_{66} a_{88}+a_{55} a_{22} a_{66} a_{77}+a_{55} a_{11} a_{22} a_{77}+a_{55} a_{61} a_{86} a_{18}-a_{55} a_{61} a_{22} a_{16}-a_{55} a_{81} a_{18} a_{77}-a_{55} a_{81} a_{18} a_{66} \\
& -a_{55} a_{81} a_{22} a_{18}-a_{44} a_{22} a_{76} a_{67}-a_{44} a_{11} a_{76} a_{67}+a_{44} a_{11} a_{77} a_{88}+a_{44} a_{11} a_{66} a_{88}+a_{44} a_{11} a_{66} a_{77}+a_{44} a_{11} a_{22} a_{88}+a_{44} a_{11} a_{22} a_{66} \\
& -a_{44} a_{61} a_{16} a_{88}-a_{44} a_{61} a_{16} a_{77}+a_{44} a_{66} a_{77} a_{88}-a_{44} a_{76} a_{67} a_{88}+a_{44} a_{86} a_{67} a_{78}+a_{44} a_{22} a_{77} a_{88}+a_{44} a_{22} a_{66} a_{88}+a_{44} a_{22} a_{66} a_{77} \\
& +a_{33} a_{44} a_{55} a_{11}-a_{33} a_{61} a_{22} a_{16}-a_{33} a_{81} a_{18} a_{77}+a_{33} a_{55} a_{22} a_{77}-a_{33} a_{81} a_{18} a_{66}-a_{33} a_{81} a_{22} a_{18}+a_{33} a_{55} a_{77} a_{88}+a_{33} a_{55} a_{11} a_{66} \\
& +a_{33} a_{55} a_{22} a_{88}-a_{33} a_{55} a_{76} a_{67}+a_{33} a_{55} a_{66} a_{88}+a_{33} a_{55} a_{11} a_{88}+a_{33} a_{55} a_{66} a_{77}-a_{33} a_{55} a_{61} a_{16}+a_{33} a_{55} a_{22} a_{66}+a_{33} a_{55} a_{11} a_{77} \\
& +a_{33} a_{55} a_{11} a_{22}-a_{33} a_{55} a_{81} a_{18}+a_{33} a_{44} a_{22} a_{77}+a_{33} a_{44} a_{11} a_{66}+a_{33} a_{44} a_{77} a_{88}+a_{33} a_{44} a_{11} a_{88}-a_{33} a_{44} a_{76} a_{67}+a_{33} a_{44} a_{22} a_{88} \\
& +a_{33} a_{44} a_{66} a_{77}+a_{33} a_{44} a_{66} a_{88}+a_{33} a_{44} a_{22} a_{66}-a_{33} a_{44} a_{61} a_{16}+a_{33} a_{44} a_{11} a_{77}+a_{33} a_{44} a_{11} a_{22}+a_{33} a_{44} a_{55} a_{88}-a_{33} a_{44} a_{81} a_{18} \\
& +a_{33} a_{44} a_{55} a_{77}+a_{33} a_{44} a_{55} a_{66}+a_{33} a_{44} a_{55} a_{22}+a_{51} a_{15} a_{76} a_{67}-a_{51} a_{15} a_{77} a_{88}-a_{61} a_{22} a_{16} a_{77}+a_{61} a_{22} a_{86} a_{18}+a_{11} a_{22} a_{66} a_{77} \\
& -a_{11} a_{22} a_{76} a_{67}-a_{81} a_{22} a_{18} a_{66}+a_{51} a_{65} a_{16} a_{77}
\end{aligned}
$$

$A_{5}=-a_{55} a_{22} a_{66} a_{77} a_{88}+a_{81} a_{22} a_{16} a_{67} a_{78}-a_{81} a_{22} a_{76} a_{18} a_{67}+a_{81} a_{22} a_{18} a_{66} a_{77}-a_{55} a_{22} a_{86} a_{67} a_{78}-a_{55} a_{11} a_{66} a_{77} a_{88}$ $-a_{44} a_{55} a_{11} a_{66} a_{77}+a_{44} a_{55} a_{81} a_{22} a_{18}-a_{44} a_{22} a_{66} a_{77} a_{88}-a_{44} a_{11} a_{86} a_{67} a_{78}-a_{44} a_{11} a_{22} a_{66} a_{77}+a_{44} a_{11} a_{76} a_{67} a_{88}$ $+a_{44} a_{22} a_{76} a_{67} a_{88}+a_{55} a_{22} a_{76} a_{67} a_{88}-a_{44} a_{11} a_{66} a_{77} a_{88}-a_{44} a_{11} a_{22} a_{66} a_{88}+a_{44} a_{61} a_{16} a_{77} a_{88}+a_{44} a_{81} a_{22} a_{18} a_{66}$ $+a_{44} a_{61} a_{22} a_{16} a_{77}+a_{44} a_{11} a_{22} a_{76} a_{67}+a_{44} a_{81} a_{18} a_{66} a_{77}+a_{44} a_{55} a_{61} a_{16} a_{88}-a_{44} a_{55} a_{11} a_{22} a_{66}+a_{44} a_{81} a_{22} a_{18} a_{77}$ $+a_{44} a_{55} a_{22} a_{76} a_{67}-a_{44} a_{61} a_{22} a_{86} a_{18}-a_{44} a_{55} a_{11} a_{77} a_{88}+a_{44} a_{81} a_{16} a_{67} a_{78}-a_{44} a_{11} a_{22} a_{77} a_{88}-a_{44} a_{55} a_{11} a_{22} a_{88}$ $-a_{44} a_{61} a_{86} a_{18} a_{77}-a_{44} a_{81} a_{76} a_{18} a_{67}+a_{44} a_{55} a_{81} a_{18} a_{66}-a_{44} a_{22} a_{86} a_{67} a_{78}-a_{44} a_{55} a_{11} a_{66} a_{88}+a_{44} a_{55} a_{11} a_{76} a_{67}$ $+a_{44} a_{61} a_{22} a_{16} a_{88}+a_{44} a_{55} a_{81} a_{18} a_{77}+a_{44} a_{55} a_{61} a_{22} a_{16}-a_{44} a_{55} a_{61} a_{86} a_{18}-a_{44} a_{55} a_{11} a_{22} a_{77}-a_{44} a_{55} a_{22} a_{66} a_{77}$ $-a_{44} a_{55} a_{22} a_{66} a_{88}-a_{44} a_{55} a_{22} a_{77} a_{88}-a_{44} a_{55} a_{86} a_{67} a_{78}+a_{44} a_{55} a_{76} a_{67} a_{88}-a_{44} a_{55} a_{66} a_{77} a_{88}+a_{44} a_{55} a_{61} a_{16} a_{77}$ $+a_{33} a_{81} a_{22} a_{18} a_{77}-a_{55} a_{61} a_{86} a_{18} a_{77}+a_{33} a_{81} a_{22} a_{18} a_{66}+a_{33} a_{11} a_{76} a_{67} a_{88}-a_{33} a_{11} a_{86} a_{67} a_{78}+a_{33} a_{81} a_{16} a_{67} a_{78}$ $-a_{33} a_{61} a_{22} a_{86} a_{18}-a_{33} a_{11} a_{22} a_{66} a_{77}-a_{33} a_{11} a_{22} a_{66} a_{88}-a_{33} a_{11} a_{22} a_{77} a_{88}+a_{33} a_{11} a_{22} a_{76} a_{67}-a_{33} a_{81} a_{76} a_{18} a_{67}$ $+a_{33} a_{81} a_{18} a_{66} a_{77}-a_{33} a_{61} a_{86} a_{18} a_{77}+a_{33} a_{61} a_{16} a_{77} a_{88}-a_{33} a_{11} a_{66} a_{77} a_{88}-a_{33} a_{22} a_{86} a_{67} a_{78}+a_{33} a_{22} a_{76} a_{67} a_{88}$ $-a_{33} a_{22} a_{66} a_{77} a_{88}+a_{33} a_{61} a_{22} a_{16} a_{77}+a_{33} a_{61} a_{22} a_{16} a_{88}+a_{51} a_{65} a_{86} a_{18} a_{77}+a_{51} a_{15} a_{66} a_{77} a_{88}+a_{51} a_{75} a_{16} a_{67} a_{88}$ $-a_{51} a_{85} a_{18} a_{66} a_{77}+a_{51} a_{85} a_{76} a_{18} a_{67}-a_{51} a_{85} a_{16} a_{67} a_{78}-a_{51} a_{75} a_{86} a_{18} a_{67}-a_{51} a_{65} a_{16} a_{77} a_{88}-a_{51} a_{15} a_{76} a_{67} a_{88}$ $+a_{51} a_{15} a_{86} a_{67} a_{78}+a_{51} a_{33} a_{22} a_{15} a_{66}-a_{51} a_{33} a_{22} a_{85} a_{18}+a_{51} a_{33} a_{65} a_{86} a_{18}+a_{51} a_{33} a_{15} a_{66} a_{88}+a_{51} a_{33} a_{75} a_{16} a_{67}$ 


$$
\begin{aligned}
& -a_{51} a_{33} a_{85} a_{18} a_{77}-a_{51} a_{33} a_{85} a_{18} a_{66}+a_{51} a_{33} a_{15} a_{66} a_{77}-a_{51} a_{33} a_{65} a_{16} a_{88}-a_{51} a_{33} a_{65} a_{16} a_{77}-a_{51} a_{33} a_{15} a_{76} a_{67} \\
& +a_{51} a_{33} a_{15} a_{77} a_{88}-a_{51} a_{44} a_{22} a_{65} a_{16}+a_{51} a_{44} a_{22} a_{15} a_{77}+a_{51} a_{44} a_{22} a_{15} a_{88}+a_{51} a_{44} a_{22} a_{15} a_{66}-a_{51} a_{44} a_{22} a_{85} a_{18} \\
& +a_{51} a_{44} a_{65} a_{86} a_{18}+a_{51} a_{33} a_{22} a_{15} a_{77}+a_{51} a_{33} a_{22} a_{15} a_{88}-a_{51} a_{33} a_{44} a_{85} a_{18}-a_{51} a_{33} a_{22} a_{65} a_{16}+a_{33} a_{55} a_{81} a_{18} a_{66} \\
& +a_{33} a_{55} a_{81} a_{18} a_{77}+a_{33} a_{55} a_{61} a_{22} a_{16}-a_{33} a_{55} a_{61} a_{86} a_{18}-a_{33} a_{55} a_{11} a_{22} a_{77}-a_{33} a_{55} a_{22} a_{66} a_{77}-a_{33} a_{55} a_{22} a_{66} a_{88} \\
& -a_{33} a_{55} a_{22} a_{77} a_{88}-a_{33} a_{55} a_{86} a_{67} a_{78}+a_{33} a_{55} a_{76} a_{67} a_{88}-a_{33} a_{55} a_{66} a_{77} a_{88}+a_{33} a_{55} a_{61} a_{16} a_{77}+a_{33} a_{55} a_{61} a_{16} a_{88} \\
& -a_{33} a_{55} a_{11} a_{22} a_{66}-a_{33} a_{55} a_{11} a_{22} a_{88}+a_{33} a_{55} a_{11} a_{76} a_{67}-a_{33} a_{55} a_{11} a_{66} a_{77}-a_{33} a_{55} a_{11} a_{66} a_{88}-a_{33} a_{55} a_{11} a_{77} a_{88} \\
& +a_{33} a_{55} a_{22} a_{76} a_{67}+a_{51} a_{22} a_{15} a_{77} a_{88}-a_{51} a_{22} a_{15} a_{76} a_{67}-a_{51} a_{22} a_{65} a_{16} a_{77}-a_{51} a_{22} a_{65} a_{16} a_{88}-a_{51} a_{22} a_{85} a_{18} a_{77} \\
& +a_{51} a_{22} a_{75} a_{16} a_{67}-a_{33} a_{44} a_{55} a_{22} a_{88}-a_{33} a_{44} a_{55} a_{77} a_{88}-a_{33} a_{44} a_{55} a_{11} a_{66}-a_{33} a_{44} a_{55} a_{22} a_{77}+a_{33} a_{44} a_{81} a_{22} a_{18} \\
& +a_{33} a_{44} a_{81} a_{18} a_{66}+a_{33} a_{44} a_{81} a_{18} a_{77}+a_{33} a_{44} a_{61} a_{22} a_{16}-a_{33} a_{44} a_{61} a_{86} a_{18}-a_{33} a_{44} a_{11} a_{22} a_{77}-a_{33} a_{44} a_{22} a_{66} a_{77} \\
& -a_{33} a_{44} a_{22} a_{66} a_{88}-a_{33} a_{44} a_{22} a_{77} a_{88}-a_{33} a_{44} a_{86} a_{67} a_{78}+a_{33} a_{44} a_{76} a_{67} a_{88}-a_{33} a_{44} a_{66} a_{77} a_{88}+a_{33} a_{44} a_{61} a_{16} a_{77} \\
& +a_{33} a_{44} a_{61} a_{16} a_{88}-a_{33} a_{44} a_{11} a_{22} a_{66}-a_{33} a_{44} a_{11} a_{22} a_{88}+a_{33} a_{44} a_{11} a_{76} a_{67}-a_{33} a_{44} a_{11} a_{66} a_{77}-a_{33} a_{44} a_{11} a_{66} a_{88} \\
& -a_{33} a_{44} a_{11} a_{77} a_{88}+a_{33} a_{44} a_{22} a_{76} a_{67}+a_{33} a_{55} a_{81} a_{22} a_{18}+a_{51} a_{44} a_{15} a_{66} a_{88}+a_{51} a_{44} a_{75} a_{16} a_{67}-a_{51} a_{44} a_{85} a_{18} a_{77} \\
& -a_{51} a_{44} a_{85} a_{18} a_{66}+a_{51} a_{44} a_{15} a_{66} a_{77}-a_{51} a_{44} a_{65} a_{16} a_{88}-a_{51} a_{44} a_{65} a_{16} a_{77}-a_{51} a_{44} a_{15} a_{76} a_{67}+a_{51} a_{44} a_{15} a_{77} a_{88} \\
& +a_{51} a_{22} a_{65} a_{86} a_{18}+a_{51} a_{22} a_{15} a_{66} a_{88}-a_{33} a_{44} a_{55} a_{11} a_{88}+a_{33} a_{44} a_{55} a_{76} a_{67}-a_{33} a_{44} a_{55} a_{66} a_{77}-a_{33} a_{44} a_{55} a_{22} a_{66} \\
& -a_{33} a_{44} a_{55} a_{66} a_{88}-a_{33} a_{44} a_{55} a_{11} a_{77}+a_{33} a_{44} a_{55} a_{61} a_{16}-a_{33} a_{44} a_{55} a_{11} a_{22}+a_{33} a_{44} a_{55} a_{81} a_{18}+a_{55} a_{61} a_{22} a_{16} a_{77} \\
& -a_{11} a_{22} a_{66} a_{77} a_{88}+a_{55} a_{61} a_{22} a_{16} a_{88}+a_{61} a_{22} a_{16} a_{77} a_{88}-a_{51} a_{22} a_{85} a_{18} a_{66}+a_{51} a_{22} a_{15} a_{66} a_{77}-a_{61} a_{22} a_{86} a_{18} a_{77} \\
& +a_{55} a_{11} a_{22} a_{76} a_{67}-a_{55} a_{81} a_{76} a_{18} a_{67}+a_{55} a_{81} a_{18} a_{66} a_{77}+a_{55} a_{81} a_{16} a_{67} a_{78}-a_{55} a_{61} a_{22} a_{86} a_{18}-a_{55} a_{11} a_{22} a_{66} a_{77} \\
& -a_{55} a_{11} a_{22} a_{66} a_{88}-a_{55} a_{11} a_{22} a_{77} a_{88}-a_{55} a_{11} a_{86} a_{67} a_{78}+a_{55} a_{11} a_{76} a_{67} a_{88}+a_{55} a_{81} a_{22} a_{18} a_{66}+a_{55} a_{81} a_{22} a_{18} a_{77} \\
& +a_{51} a_{33} a_{44} a_{22} a_{15}-a_{51} a_{33} a_{44} a_{65} a_{16}+a_{51} a_{33} a_{44} a_{15} a_{77}+a_{51} a_{33} a_{44} a_{15} a_{88}+a_{51} a_{33} a_{44} a_{15} a_{66}-a_{11} a_{22} a_{86} a_{67} a_{78} \\
& +a_{11} a_{22} a_{76} a_{67} a_{88}+a_{55} a_{61} a_{16} a_{77} a_{88} \text {, }
\end{aligned}
$$

$A_{6}=a_{51} a_{33} a_{85} a_{16} a_{67} a_{78}+a_{51} a_{33} a_{75} a_{86} a_{18} a_{67}+a_{51} a_{33} a_{22} a_{65} a_{16} a_{88}-a_{51} a_{33} a_{85} a_{76} a_{18} a_{67}-a_{51} a_{33} a_{75} a_{16} a_{67} a_{88}$ $+a_{51} a_{33} a_{85} a_{18} a_{66} a_{77}+a_{51} a_{33} a_{22} a_{65} a_{16} a_{77}+a_{51} a_{33} a_{22} a_{15} a_{76} a_{67}-a_{51} a_{33} a_{22} a_{15} a_{77} a_{88}-a_{51} a_{33} a_{65} a_{86} a_{18} a_{77}$ $-a_{51} a_{33} a_{15} a_{66} a_{77} a_{88}-a_{51} a_{33} a_{22} a_{15} a_{66} a_{77}+a_{51} a_{33} a_{22} a_{85} a_{18} a_{66}+a_{51} a_{33} a_{22} a_{85} a_{18} a_{77}-a_{51} a_{33} a_{22} a_{75} a_{16} a_{67}$ $+a_{51} a_{22} a_{65} a_{16} a_{77} a_{88}+a_{51} a_{22} a_{15} a_{76} a_{67} a_{88}+a_{51} a_{44} a_{22} a_{65} a_{16} a_{88}+a_{51} a_{44} a_{22} a_{65} a_{16} a_{77}-a_{51} a_{33} a_{22} a_{15} a_{66} a_{88}$ $-a_{51} a_{33} a_{22} a_{65} a_{86} a_{18}-a_{51} a_{33} a_{44} a_{75} a_{16} a_{67}+a_{51} a_{33} a_{44} a_{85} a_{18} a_{77}+a_{51} a_{33} a_{44} a_{85} a_{18} a_{66}-a_{51} a_{33} a_{44} a_{15} a_{66} a_{77}$ $+a_{51} a_{33} a_{44} a_{65} a_{16} a_{88}+a_{51} a_{33} a_{44} a_{65} a_{16} a_{77}-a_{51} a_{22} a_{75} a_{16} a_{67} a_{88}+a_{51} a_{22} a_{85} a_{18} a_{66} a_{77}+a_{51} a_{44} a_{85} a_{16} a_{67} a_{78}$ $-a_{51} a_{44} a_{75} a_{16} a_{67} a_{88}+a_{51} a_{44} a_{85} a_{18} a_{66} a_{77}-a_{51} a_{44} a_{85} a_{76} a_{18} a_{67}-a_{51} a_{33} a_{44} a_{65} a_{86} a_{18}-a_{51} a_{44} a_{85} a_{76} a_{18} a_{67}$ $-a_{51} a_{44} a_{85} a_{76} a_{18} a_{67}-a_{51} a_{33} a_{44} a_{65} a_{86} a_{18}+a_{51} a_{33} a_{44} a_{15} a_{76} a_{67}-a_{51} a_{33} a_{44} a_{15} a_{77} a_{88}-a_{51} a_{33} a_{44} a_{15} a_{66} a_{88}$ $+a_{51} a_{33} a_{44} a_{22} a_{85} a_{18}-a_{55} a_{81} a_{22} a_{18} a_{66} a_{77}-a_{51} a_{22} a_{15} a_{86} a_{67} a_{78}-a_{51} a_{33} a_{44} a_{22} a_{15} a_{88}-a_{51} a_{33} a_{44} a_{22} a_{15} a_{77}$ $+a_{51} a_{33} a_{44} a_{22} a_{65} a_{16}-a_{51} a_{33} a_{44} a_{22} a_{15} a_{66}+a_{55} a_{61} a_{22} a_{86} a_{18} a_{77}-a_{55} a_{61} a_{22} a_{16} a_{77} a_{88}+a_{55} a_{11} a_{22} a_{66} a_{77} a_{88}$ $-a_{55} a_{81} a_{22} a_{16} a_{67} a_{78}+a_{55} a_{81} a_{22} a_{76} a_{18} a_{67}-a_{44} a_{81} a_{22} a_{18} a_{66} a_{77}-a_{55} a_{11} a_{22} a_{76} a_{67} a_{88}+a_{55} a_{11} a_{22} a_{86} a_{67} a_{78}$ $+a_{44} a_{81} a_{22} a_{76} a_{18} a_{67}+a_{44} a_{11} a_{22} a_{66} a_{77} a_{88}-a_{44} a_{81} a_{22} a_{16} a_{67} a_{78}+a_{44} a_{61} a_{22} a_{86} a_{18} a_{77}-a_{44} a_{61} a_{22} a_{16} a_{77} a_{88}$ $+a_{44} a_{11} a_{22} a_{86} a_{67} a_{78}-a_{44} a_{11} a_{22} a_{76} a_{67} a_{88}-a_{44} a_{55} a_{81} a_{22} a_{18} a_{77}-a_{44} a_{55} a_{11} a_{76} a_{67} a_{88}-a_{44} a_{55} a_{81} a_{22} a_{18} a_{66}$ $+a_{44} a_{55} a_{61} a_{22} a_{86} a_{18}+a_{44} a_{55} a_{11} a_{22} a_{66} a_{77}+a_{44} a_{55} a_{11} a_{22} a_{77} a_{88}+a_{44} a_{55} a_{11} a_{22} a_{66} a_{88}+a_{44} a_{55} a_{11} a_{86} a_{67} a_{78}$ $-a_{44} a_{55} a_{81} a_{16} a_{67} a_{78}-a_{44} a_{55} a_{81} a_{18} a_{66} a_{77}+a_{44} a_{55} a_{81} a_{76} a_{18} a_{67}+a_{44} a_{55} a_{61} a_{86} a_{18} a_{77}-a_{44} a_{55} a_{61} a_{16} a_{77} a_{88}$ $-a_{44} a_{55} a_{11} a_{22} a_{76} a_{67}+a_{44} a_{55} a_{22} a_{66} a_{77} a_{88}-a_{44} a_{55} a_{61} a_{22} a_{16} a_{77}-a_{44} a_{55} a_{61} a_{22} a_{16} a_{88}+a_{44} a_{55} a_{11} a_{66} a_{77} a_{88}$ $+a_{44} a_{55} a_{22} a_{86} a_{67} a_{78}-a_{44} a_{55} a_{22} a_{76} a_{67} a_{88}-a_{33} a_{81} a_{22} a_{18} a_{66} a_{77}-a_{33} a_{55} a_{81} a_{22} a_{18} a_{77}+a_{33} a_{11} a_{22} a_{86} a_{67} a_{78}$ $-a_{33} a_{11} a_{22} a_{76} a_{67} a_{88}+a_{33} a_{61} a_{22} a_{86} a_{18} a_{77}-a_{33} a_{61} a_{22} a_{16} a_{77} a_{88}+a_{33} a_{11} a_{22} a_{66} a_{77} a_{88}-a_{33} a_{81} a_{22} a_{16} a_{67} a_{78}$ $+a_{33} a_{81} a_{22} a_{76} a_{18} a_{67}+a_{33} a_{55} a_{11} a_{86} a_{67} a_{78}-a_{33} a_{55} a_{11} a_{76} a_{67} a_{88}-a_{33} a_{55} a_{81} a_{22} a_{18} a_{66}+a_{33} a_{55} a_{11} a_{22} a_{66} a_{88}$ $+a_{33} a_{55} a_{11} a_{22} a_{77} a_{88}+a_{33} a_{55} a_{61} a_{22} a_{86} a_{18}+a_{33} a_{55} a_{11} a_{22} a_{66} a_{77}-a_{33} a_{55} a_{81} a_{16} a_{67} a_{78}+a_{33} a_{55} a_{81} a_{76} a_{18} a_{67}$ 


$$
\begin{aligned}
& -a_{33} a_{55} a_{81} a_{18} a_{66} a_{77}-a_{33} a_{55} a_{11} a_{22} a_{76} a_{67}-a_{33} a_{55} a_{61} a_{16} a_{77} a_{88}-a_{33} a_{55} a_{61} a_{22} a_{16} a_{88}+a_{33} a_{55} a_{61} a_{86} a_{18} a_{77} \\
& -a_{33} a_{44} a_{55} a_{22} a_{76} a_{67}-a_{51} a_{44} a_{22} a_{15} a_{77} a_{88}-a_{51} a_{44} a_{65} a_{86} a_{18} a_{77}-a_{51} a_{44} a_{15} a_{66} a_{77} a_{88}+a_{33} a_{55} a_{22} a_{66} a_{77} a_{88} \\
& -a_{33} a_{55} a_{61} a_{22} a_{16} a_{77}-a_{33} a_{44} a_{81} a_{22} a_{18} a_{77}+a_{33} a_{55} a_{11} a_{66} a_{77} a_{88}+a_{33} a_{55} a_{22} a_{86} a_{67} a_{78}-a_{33} a_{55} a_{22} a_{76} a_{67} a_{88} \\
& -a_{33} a_{44} a_{81} a_{22} a_{18} a_{66}+a_{33} a_{44} a_{11} a_{22} a_{77} a_{88}+a_{33} a_{44} a_{11} a_{86} a_{67} a_{78}-a_{33} a_{44} a_{11} a_{76} a_{67} a_{88}+a_{33} a_{44} a_{11} a_{22} a_{66} a_{88} \\
& -a_{33} a_{44} a_{81} a_{16} a_{67} a_{78}+a_{33} a_{44} a_{61} a_{22} a_{86} a_{18}+a_{33} a_{44} a_{11} a_{22} a_{66} a_{77}+a_{33} a_{44} a_{81} a_{76} a_{18} a_{67}-a_{33} a_{44} a_{81} a_{18} a_{66} a_{77} \\
& -a_{33} a_{44} a_{61} a_{16} a_{77} a_{88}-a_{33} a_{44} a_{11} a_{22} a_{76} a_{67}-a_{33} a_{44} a_{61} a_{22} a_{16} a_{77}-a_{33} a_{44} a_{61} a_{22} a_{16} a_{88}+a_{33} a_{44} a_{55} a_{11} a_{22} a_{66} \\
& +a_{33} a_{44} a_{55} a_{66} a_{77} a_{88}-a_{33} a_{44} a_{55} a_{61} a_{16} a_{77}-a_{33} a_{44} a_{55} a_{61} a_{16} a_{88}+a_{33} a_{44} a_{55} a_{11} a_{22} a_{77}+a_{33} a_{44} a_{55} a_{22} a_{66} a_{77} \\
& +a_{33} a_{44} a_{55} a_{22} a_{66} a_{88}+a_{33} a_{44} a_{55} a_{22} a_{77} a_{88}+a_{33} a_{44} a_{55} a_{86} a_{67} a_{78}+a_{33} a_{44} a_{55} a_{61} a_{86} a_{18}-a_{33} a_{44} a_{55} a_{61} a_{22} a_{16} \\
& -a_{33} a_{44} a_{55} a_{81} a_{18} a_{77}-a_{33} a_{44} a_{55} a_{81} a_{22} a_{18}-a_{33} a_{44} a_{55} a_{81} a_{18} a_{66}+a_{33} a_{44} a_{22} a_{66} a_{77} a_{88}+a_{33} a_{44} a_{61} a_{86} a_{18} a_{77} \\
& +a_{33} a_{44} a_{11} a_{66} a_{77} a_{88}+a_{33} a_{44} a_{22} a_{86} a_{67} a_{78}-a_{51} a_{44} a_{22} a_{65} a_{86} a_{18}+a_{51} a_{33} a_{15} a_{76} a_{67} a_{88}-a_{51} a_{44} a_{22} a_{75} a_{16} a_{67} \\
& -a_{51} a_{33} a_{15} a_{86} a_{67} a_{78}+a_{51} a_{33} a_{65} a_{16} a_{77} a_{88}-a_{51} a_{44} a_{22} a_{15} a_{66} a_{88}+a_{33} a_{44} a_{55} a_{11} a_{66} a_{77}+a_{33} a_{44} a_{55} a_{11} a_{22} a_{88} \\
& -a_{33} a_{44} a_{55} a_{11} a_{76} a_{67}+a_{33} a_{44} a_{55} a_{11} a_{66} a_{88}+a_{33} a_{44} a_{55} a_{11} a_{77} a_{88}+a_{51} a_{44} a_{22} a_{15} a_{76} a_{67}+a_{51} a_{44} a_{22} a_{85} a_{18} a_{66} \\
& -a_{51} a_{44} a_{22} a_{15} a_{66} a_{77}+a_{51} a_{44} a_{22} a_{85} a_{18} a_{77}-a_{33} a_{44} a_{55} a_{76} a_{67} a_{88}-a_{33} a_{44} a_{22} a_{76} a_{67} a_{88}+a_{51} a_{44} a_{75} a_{86} a_{18} a_{67} \\
& +a_{51} a_{44} a_{65} a_{16} a_{77} a_{88}+a_{51} a_{44} a_{15} a_{76} a_{67} a_{88}-a_{51} a_{44} a_{15} a_{86} a_{67} a_{78}-a_{51} a_{22} a_{65} a_{86} a_{18} a_{77}-a_{51} a_{22} a_{15} a_{66} a_{77} a_{88} \\
& -a_{51} a_{22} a_{85} a_{76} a_{18} a_{67}+a_{51} a_{22} a_{85} a_{16} a_{67} a_{78}+a_{51} a_{22} a_{75} a_{86} a_{18} a_{67} \text {, } \\
& A_{7}=a_{51} a_{44} a_{22} a_{15} a_{86} a_{67} a_{78}-a_{51} a_{33} a_{44} a_{22} a_{15} a_{76} a_{67}-a_{51} a_{33} a_{44} a_{22} a_{65} a_{16} a_{77}+a_{33} a_{44} a_{55} a_{81} a_{22} a_{18} a_{77} \\
& +a_{33} a_{44} a_{55} a_{81} a_{22} a_{18} a_{66}+a_{33} a_{44} a_{55} a_{11} a_{76} a_{67} a_{88}-a_{33} a_{44} a_{61} a_{22} a_{86} a_{18} a_{77}-a_{33} a_{44} a_{11} a_{22} a_{86} a_{67} a_{78} \\
& +a_{33} a_{44} a_{11} a_{22} a_{76} a_{67} a_{88}-a_{51} a_{44} a_{22} a_{75} a_{86} a_{18} a_{67}+a_{51} a_{33} a_{44} a_{15} a_{66} a_{77} a_{88}-a_{51} a_{44} a_{22} a_{15} a_{76} a_{67} a_{88} \\
& +a_{51} a_{33} a_{22} a_{15} a_{66} a_{77} a_{88}+a_{33} a_{55} a_{81} a_{22} a_{16} a_{67} a_{78}-a_{51} a_{33} a_{22} a_{15} a_{76} a_{67} a_{88}+a_{51} a_{33} a_{44} a_{65} a_{86} a_{18} a_{77} \\
& -a_{33} a_{44} a_{55} a_{11} a_{22} a_{77} a_{88}+a_{44} a_{55} a_{61} a_{22} a_{16} a_{77} a_{88}-a_{44} a_{55} a_{61} a_{22} a_{86} a_{18} a_{77}+a_{51} a_{33} a_{44} a_{22} a_{15} a_{66} a_{88} \\
& +a_{51} a_{33} a_{44} a_{22} a_{75} a_{16} a_{67}+a_{33} a_{44} a_{81} a_{22} a_{18} a_{66} a_{77}-a_{33} a_{44} a_{81} a_{22} a_{76} a_{18} a_{67}+a_{33} a_{44} a_{81} a_{22} a_{16} a_{67} a_{78} \\
& -a_{33} a_{44} a_{11} a_{22} a_{66} a_{77} a_{88}-a_{33} a_{55} a_{11} a_{22} a_{66} a_{77} a_{88}-a_{44} a_{55} a_{81} a_{22} a_{76} a_{18} a_{67}+a_{44} a_{55} a_{81} a_{22} a_{16} a_{67} a_{78} \\
& -a_{44} a_{55} a_{11} a_{22} a_{66} a_{77} a_{88}-a_{51} a_{33} a_{44} a_{22} a_{65} a_{16} a_{88}+a_{51} a_{33} a_{22} a_{65} a_{86} a_{18} a_{77}-a_{51} a_{33} a_{22} a_{85} a_{18} a_{66} a_{77} \\
& -a_{51} a_{33} a_{22} a_{75} a_{86} a_{18} a_{67}+a_{51} a_{33} a_{44} a_{22} a_{15} a_{77} a_{88}+a_{51} a_{33} a_{44} a_{22} a_{65} a_{86} a_{18}-a_{51} a_{33} a_{22} a_{85} a_{16} a_{67} a_{78} \\
& +a_{51} a_{33} a_{22} a_{75} a_{16} a_{67} a_{88}-a_{51} a_{44} a_{22} a_{65} a_{16} a_{77} a_{88}+a_{51} a_{33} a_{44} a_{75} a_{16} a_{67} a_{88}-a_{51} a_{33} a_{44} a_{85} a_{16} a_{67} a_{78} \\
& -a_{51} a_{33} a_{44} a_{22} a_{85} a_{18} a_{77}-a_{51} a_{33} a_{44} a_{22} a_{85} a_{18} a_{66}-a_{51} a_{44} a_{22} a_{85} a_{18} a_{66} a_{77}+a_{51} a_{33} a_{44} a_{85} a_{76} a_{18} a_{67} \\
& +a_{51} a_{33} a_{22} a_{15} a_{86} a_{67} a_{78}+a_{51} a_{44} a_{22} a_{75} a_{16} a_{67} a_{88}-a_{51} a_{44} a_{22} a_{85} a_{16} a_{67} a_{78}+a_{51} a_{33} a_{22} a_{85} a_{76} a_{18} a_{67} \\
& -a_{33} a_{44} a_{55} a_{11} a_{86} a_{67} a_{78}+a_{51} a_{44} a_{22} a_{15} a_{66} a_{77} a_{88}+a_{51} a_{44} a_{22} a_{85} a_{76} a_{18} a_{67}-a_{51} a_{33} a_{44} a_{75} a_{86} a_{18} a_{67} \\
& -a_{51} a_{33} a_{44} a_{65} a_{16} a_{77} a_{88}+a_{44} a_{55} a_{81} a_{22} a_{18} a_{66} a_{77}+a_{51} a_{33} a_{44} a_{22} a_{15} a_{66} a_{77}-a_{33} a_{44} a_{55} a_{11} a_{22} a_{66} a_{77} \\
& -a_{33} a_{44} a_{55} a_{11} a_{22} a_{66} a_{88}+a_{33} a_{44} a_{55} a_{61} a_{22} a_{16} a_{77}-a_{33} a_{44} a_{55} a_{61} a_{22} a_{86} a_{18}+a_{33} a_{44} a_{55} a_{81} a_{16} a_{67} a_{78} \\
& +a_{33} a_{44} a_{55} a_{81} a_{18} a_{66} a_{77}-a_{33} a_{44} a_{55} a_{81} a_{76} a_{18} a_{67}+a_{33} a_{44} a_{55} a_{11} a_{22} a_{76} a_{67}+a_{33} a_{44} a_{55} a_{61} a_{16} a_{77} a_{88} \\
& -a_{33} a_{44} a_{55} a_{61} a_{86} a_{18} a_{77}+a_{33} a_{44} a_{55} a_{61} a_{22} a_{16} a_{88}+a_{33} a_{55} a_{61} a_{22} a_{16} a_{77} a_{88}-a_{33} a_{55} a_{61} a_{22} a_{86} a_{18} a_{77} \\
& +a_{33} a_{55} a_{11} a_{22} a_{76} a_{67} a_{88}-a_{33} a_{55} a_{11} a_{22} a_{86} a_{67} a_{78}-a_{33} a_{44} a_{55} a_{22} a_{66} a_{77} a_{88}+a_{33} a_{44} a_{55} a_{22} a_{76} a_{67} a_{88} \\
& -a_{33} a_{44} a_{55} a_{22} a_{86} a_{67} a_{78}-a_{33} a_{44} a_{55} a_{11} a_{66} a_{77} a_{88}+a_{33} a_{55} a_{81} a_{22} a_{18} a_{66} a_{77}-a_{33} a_{55} a_{81} a_{22} a_{76} a_{18} a_{67} \\
& -a_{51} a_{33} a_{44} a_{15} a_{76} a_{67} a_{88}+a_{51} a_{44} a_{22} a_{65} a_{86} a_{18} a_{77}+a_{51} a_{33} a_{44} a_{15} a_{86} a_{67} a_{78}+a_{33} a_{44} a_{61} a_{22} a_{16} a_{77} a_{88} \\
& +a_{44} a_{55} a_{11} a_{22} a_{76} a_{67} a_{88}-a_{44} a_{55} a_{11} a_{22} a_{86} a_{67} a_{78}-a_{51} a_{33} a_{44} a_{85} a_{18} a_{66} a_{77}-a_{51} a_{33} a_{22} a_{65} a_{16} a_{77} a_{88} \text {, } \\
& A_{8}=-a_{51} a_{33} a_{44} a_{22} a_{85} a_{76} a_{18} a_{67}+a_{51} a_{33} a_{44} a_{22} a_{85} a_{16} a_{67} a_{78}+a_{51} a_{33} a_{44} a_{22} a_{75} a_{86} a_{18} a_{67}+a_{51} a_{33} a_{44} a_{22} a_{65} a_{16} a_{77} a_{88} \\
& +a_{51} a_{33} a_{44} a_{22} a_{15} a_{76} a_{67} a_{88}-a_{51} a_{33} a_{44} a_{22} a_{15} a_{86} a_{67} a_{78}-a_{51} a_{33} a_{44} a_{22} a_{15} a_{66} a_{77} a_{88}-a_{51} a_{33} a_{44} a_{22} a_{75} a_{16} a_{67} a_{88} \\
& +a_{51} a_{33} a_{44} a_{22} a_{85} a_{18} a_{66} a_{77}-a_{51} a_{33} a_{44} a_{22} a_{65} a_{86} a_{18} a_{77}-a_{33} a_{44} a_{55} a_{81} a_{22} a_{16} a_{67} a_{78}+a_{33} a_{44} a_{55} a_{11} a_{22} a_{66} a_{77} a_{88} \\
& -a_{33} a_{44} a_{55} a_{61} a_{22} a_{16} a_{77} a_{88}+a_{33} a_{44} a_{55} a_{81} a_{22} a_{76} a_{18} a_{67}-a_{33} a_{44} a_{55} a_{11} a_{22} a_{76} a_{67} a_{88}+a_{33} a_{44} a_{55} a_{11} a_{22} a_{86} a_{67} a_{78} \\
& -a_{33} a_{44} a_{55} a_{81} a_{22} a_{18} a_{66} a_{77}+a_{33} a_{44} a_{55} a_{61} a_{22} a_{86} a_{18} a_{77}
\end{aligned}
$$




$$
\begin{aligned}
& B_{1}=-b_{32} a_{23} \text {, } \\
& B_{2}=-b_{32} a_{21} a_{13}+b_{32} a_{23} a_{88}+b_{32} a_{55} a_{23}+b_{32} a_{23} a_{66}+b_{32} a_{23} a_{77}+b_{32} a_{23} a_{11}+b_{32} a_{44} a_{23}-a_{43} b_{32} a_{24} \text {, } \\
& B_{3}=-a_{51} b_{32} a_{13} a_{25}+b_{32} a_{21} a_{13} a_{66}-b_{32} a_{23} a_{11} a_{88}-b_{32} a_{55} a_{23} a_{77}+b_{32} a_{81} a_{23} a_{18}-b_{32} a_{44} a_{23} a_{88}+b_{32} a_{44} a_{21} a_{13}-b_{32} a_{55} a_{23} a_{66} \\
& -b_{32} a_{44} a_{55} a_{23}-b_{32} a_{23} a_{11} a_{66}-b_{32} a_{23} a_{77} a_{88}+b_{32} a_{61} a_{23} a_{16}+a_{51} b_{32} a_{23} a_{15}-b_{32} a_{44} a_{23} a_{11}+b_{32} a_{55} a_{21} a_{13}+b_{32} a_{23} a_{76} a_{67} \\
& +b_{32} a_{21} a_{13} a_{77}-b_{32} a_{55} a_{23} a_{11}-b_{32} a_{23} a_{66} a_{77}-b_{32} a_{23} a_{11} a_{77}-b_{32} a_{44} a_{23} a_{77}-b_{32} a_{23} a_{66} a_{88}+b_{32} a_{21} a_{13} a_{88}-b_{32} a_{55} a_{23} a_{88} \\
& -b_{32} a_{44} a_{23} a_{66}+a_{43} b_{32} a_{24} a_{66}+a_{43} b_{32} a_{24} a_{77}+a_{43} b_{32} a_{24} a_{11}-a_{43} b_{32} a_{21} a_{14}+a_{43} b_{32} a_{55} a_{24}+a_{43} b_{32} a_{24} a_{88} \text {, } \\
& B_{4}=-b_{32} a_{55} a_{21} a_{13} a_{88}-b_{32} a_{55} a_{21} a_{13} a_{77}-b_{32} a_{44} a_{81} a_{23} a_{18}-b_{32} a_{44} a_{21} a_{13} a_{66}-b_{32} a_{44} a_{61} a_{23} a_{16}+b_{32} a_{44} a_{23} a_{77} a_{88}+b_{32} a_{44} a_{55} a_{23} a_{88} \\
& -a_{43} b_{32} a_{24} a_{11} a_{66}+a_{43} b_{32} a_{21} a_{14} a_{88}+a_{43} b_{32} a_{21} a_{14} a_{77}+a_{43} b_{32} a_{21} a_{14} a_{66}-a_{43} b_{32} a_{24} a_{77} a_{88}-a_{43} b_{32} a_{21} a_{64} a_{16}-a_{43} b_{32} a_{21} a_{84} a_{18} \\
& +a_{43} b_{32} a_{61} a_{24} a_{16}+a_{43} b_{32} a_{81} a_{24} a_{18}-a_{43} b_{32} a_{24} a_{66} a_{88}-a_{43} b_{32} a_{24} a_{66} a_{77}+a_{43} b_{32} a_{24} a_{76} a_{67}-a_{43} b_{32} a_{24} a_{11} a_{88}-a_{43} b_{32} a_{24} a_{11} a_{77} \\
& -a_{43} b_{32} a_{55} a_{24} a_{66}-a_{43} b_{32} a_{55} a_{24} a_{77}-a_{43} b_{32} a_{55} a_{24} a_{88}-a_{43} b_{32} a_{55} a_{24} a_{11}+a_{43} b_{32} a_{55} a_{21} a_{14}-b_{32} a_{23} a_{11} a_{76} a_{67}+b_{32} a_{23} a_{66} a_{77} a_{88} \\
& -b_{32} a_{81} a_{23} a_{18} a_{77}+b_{32} a_{23} a_{11} a_{66} a_{88}+b_{32} a_{61} a_{23} a_{86} a_{18}-b_{32} a_{23} a_{76} a_{67} a_{88}-b_{32} a_{21} a_{13} a_{77} a_{88}+b_{32} a_{21} a_{13} a_{76} a_{67}+b_{32} a_{23} a_{86} a_{67} a_{78} \\
& -b_{32} a_{81} a_{23} a_{18} a_{66}-b_{32} a_{61} a_{23} a_{16} a_{77}-b_{32} a_{44} a_{23} a_{76} a_{67}+b_{32} a_{44} a_{23} a_{11} a_{88}+b_{32} a_{44} a_{23} a_{66} a_{77}+b_{32} a_{44} a_{23} a_{11} a_{77}+b_{32} a_{44} a_{23} a_{11} a_{66} \\
& -b_{32} a_{44} a_{21} a_{13} a_{88}-b_{32} a_{44} a_{21} a_{13} a_{77}+b_{32} a_{44} a_{55} a_{23} a_{77}-b_{32} a_{44} a_{55} a_{21} a_{13}-a_{51} b_{32} a_{23} a_{15} a_{77}-a_{51} b_{32} a_{23} a_{15} a_{66}+a_{51} b_{32} a_{23} a_{65} a_{16} \\
& +a_{51} b_{32} a_{23} a_{85} a_{18}-a_{51} b_{32} a_{44} a_{23} a_{15}+a_{51} b_{32} a_{44} a_{13} a_{25}+b_{32} a_{44} a_{23} a_{66} a_{88}+b_{32} a_{44} a_{55} a_{23} a_{11}+b_{32} a_{44} a_{55} a_{23} a_{66}+b_{32} a_{23} a_{11} a_{66} a_{77} \\
& +b_{32} a_{23} a_{11} a_{77} a_{88}-b_{32} a_{61} a_{23} a_{16} a_{88}-b_{32} a_{21} a_{13} a_{66} a_{77}-b_{32} a_{21} a_{13} a_{66} a_{88}-b_{32} a_{55} a_{81} a_{23} a_{18}-b_{32} a_{55} a_{21} a_{13} a_{66}-b_{32} a_{55} a_{61} a_{23} a_{16} \\
& +a_{51} a_{43} b_{32} a_{24} a_{15}-a_{51} a_{43} b_{32} a_{14} a_{25}+a_{51} b_{32} a_{13} a_{25} a_{88}+a_{51} b_{32} a_{13} a_{25} a_{77}+a_{51} b_{32} a_{13} a_{25} a_{66}-a_{51} b_{32} a_{23} a_{15} a_{88}+b_{32} a_{55} a_{23} a_{77} a_{88} \\
& +b_{32} a_{55} a_{23} a_{66} a_{88}+b_{32} a_{55} a_{23} a_{66} a_{77}-b_{32} a_{55} a_{23} a_{76} a_{67}+b_{32} a_{55} a_{23} a_{11} a_{88}+b_{32} a_{55} a_{23} a_{11} a_{77}+b_{32} a_{55} a_{23} a_{11} a_{66} \text {, } \\
& B_{5}=-a_{43} b_{32} a_{21} a_{14} a_{66} a_{77}-a_{43} b_{32} a_{21} a_{14} a_{66} a_{88}+a_{43} b_{32} a_{21} a_{14} a_{76} a_{67}-b_{32} a_{55} a_{21} a_{13} a_{76} a_{67}+b_{32} a_{55} a_{61} a_{23} a_{16} a_{88}+b_{32} a_{44} a_{55} a_{21} a_{13} a_{77} \\
& +b_{32} a_{55} a_{23} a_{11} a_{76} a_{67}+b_{32} a_{55} a_{21} a_{13} a_{77} a_{88}+b_{32} a_{55} a_{23} a_{76} a_{67} a_{88}-b_{32} a_{55} a_{23} a_{66} a_{77} a_{88}+b_{32} a_{44} a_{81} a_{23} a_{18} a_{66}-a_{43} b_{32} a_{81} a_{24} a_{18} a_{77} \\
& +a_{43} b_{32} a_{24} a_{86} a_{67} a_{78}-a_{51} b_{32} a_{13} a_{25} a_{66} a_{77}-a_{51} b_{32} a_{13} a_{25} a_{66} a_{88}-b_{32} a_{44} a_{55} a_{23} a_{11} a_{88}+b_{32} a_{44} a_{55} a_{21} a_{13} a_{88}+b_{32} a_{44} a_{55} a_{81} a_{23} a_{18} \\
& -b_{32} a_{44} a_{55} a_{23} a_{11} a_{66}+a_{43} b_{32} a_{55} a_{24} a_{66} a_{88}+b_{32} a_{44} a_{21} a_{13} a_{66} a_{77}+b_{32} a_{44} a_{21} a_{13} a_{77} a_{88}-b_{32} a_{44} a_{23} a_{11} a_{66} a_{77}-a_{51} b_{32} a_{44} a_{23} a_{65} a_{16} \\
& +a_{51} b_{32} a_{44} a_{23} a_{15} a_{66}-b_{32} a_{44} a_{55} a_{23} a_{66} a_{77}-b_{32} a_{81} a_{23} a_{76} a_{18} a_{67}+b_{32} a_{44} a_{55} a_{21} a_{13} a_{66}-b_{32} a_{44} a_{55} a_{23} a_{77} a_{88}-b_{32} a_{23} a_{11} a_{86} a_{67} a_{78} \\
& +a_{51} a_{43} b_{32} a_{14} a_{25} a_{77}-b_{32} a_{55} a_{61} a_{23} a_{86} a_{18}-a_{51} b_{32} a_{44} a_{23} a_{85} a_{18}+b_{32} a_{44} a_{23} a_{76} a_{67} a_{88}-b_{32} a_{44} a_{23} a_{66} a_{77} a_{88}-a_{43} b_{32} a_{55} a_{21} a_{14} a_{77} \\
& -a_{43} b_{32} a_{55} a_{21} a_{14} a_{88}+a_{43} b_{32} a_{55} a_{24} a_{11} a_{66}-a_{43} b_{32} a_{21} a_{74} a_{16} a_{67}+a_{43} b_{32} a_{55} a_{21} a_{84} a_{18}+a_{43} b_{32} a_{55} a_{21} a_{64} a_{16}-a_{43} b_{32} a_{55} a_{61} a_{24} a_{16} \\
& -a_{43} b_{32} a_{55} a_{81} a_{24} a_{18}+a_{43} b_{32} a_{55} a_{24} a_{11} a_{77}+a_{43} b_{32} a_{55} a_{24} a_{11} a_{88}+b_{32} a_{81} a_{23} a_{16} a_{67} a_{78}+b_{32} a_{44} a_{61} a_{23} a_{16} a_{88}-a_{43} b_{32} a_{55} a_{24} a_{76} a_{67} \\
& +b_{32} a_{44} a_{61} a_{23} a_{16} a_{77}-b_{32} a_{44} a_{61} a_{23} a_{86} a_{18}-a_{43} b_{32} a_{24} a_{11} a_{76} a_{67}+a_{51} b_{32} a_{44} a_{23} a_{15} a_{77}+a_{51} b_{32} a_{44} a_{23} a_{15} a_{88}-a_{51} b_{32} a_{13} a_{25} a_{77} a_{88} \\
& -a_{43} b_{32} a_{24} a_{76} a_{67} a_{88}-b_{32} a_{44} a_{23} a_{86} a_{67} a_{78}-a_{51} a_{43} b_{32} a_{84} a_{25} a_{18}+a_{51} b_{32} a_{23} a_{15} a_{66} a_{77}+a_{51} b_{32} a_{23} a_{15} a_{66} a_{88}-b_{32} a_{55} a_{23} a_{11} a_{66} a_{77} \\
& +b_{32} a_{55} a_{61} a_{23} a_{16} a_{77}+a_{43} b_{32} a_{24} a_{11} a_{66} a_{77}+a_{43} b_{32} a_{24} a_{11} a_{66} a_{88}+a_{51} a_{43} b_{32} a_{14} a_{25} a_{88}+a_{43} b_{32} a_{61} a_{24} a_{86} a_{18}+a_{43} b_{32} a_{21} a_{64} a_{16} a_{88} \\
& -a_{43} b_{32} a_{21} a_{64} a_{86} a_{18}+a_{43} b_{32} a_{21} a_{64} a_{16} a_{77}-a_{43} b_{32} a_{55} a_{21} a_{14} a_{66}-b_{32} a_{44} a_{23} a_{11} a_{77} a_{88}-b_{32} a_{21} a_{13} a_{76} a_{67} a_{88}-a_{51} a_{43} b_{32} a_{24} a_{15} a_{88} \\
& +a_{51} a_{43} b_{32} a_{24} a_{65} a_{16}+b_{32} a_{81} a_{23} a_{18} a_{66} a_{77}-b_{32} a_{55} a_{23} a_{11} a_{66} a_{88}-a_{43} b_{32} a_{61} a_{24} a_{16} a_{88}+a_{43} b_{32} a_{21} a_{84} a_{18} a_{66}+a_{43} b_{32} a_{21} a_{84} a_{18} a_{77} \\
& +b_{32} a_{44} a_{23} a_{11} a_{76} a_{67}+b_{32} a_{55} a_{21} a_{13} a_{66} a_{88}-a_{51} b_{32} a_{44} a_{13} a_{25} a_{66}-a_{51} b_{32} a_{44} a_{13} a_{25} a_{77}+b_{32} a_{21} a_{13} a_{86} a_{67} a_{78}-a_{51} a_{43} b_{32} a_{64} a_{25} a_{16} \\
& +a_{51} a_{43} b_{32} a_{24} a_{85} a_{18}+a_{51} a_{43} b_{32} a_{14} a_{25} a_{66}-a_{43} b_{32} a_{21} a_{14} a_{77} a_{88}+b_{32} a_{44} a_{21} a_{13} a_{66} a_{88}+b_{32} a_{55} a_{81} a_{23} a_{18} a_{77}-b_{32} a_{44} a_{55} a_{23} a_{66} a_{88} \\
& -a_{51} a_{43} b_{32} a_{24} a_{15} a_{66}-a_{43} b_{32} a_{61} a_{24} a_{16} a_{77}+a_{51} b_{32} a_{23} a_{15} a_{77} a_{88}+a_{51} b_{32} a_{13} a_{25} a_{76} a_{67}+b_{32} a_{44} a_{55} a_{23} a_{76} a_{67}+b_{32} a_{55} a_{21} a_{13} a_{66} a_{77} \\
& -b_{32} a_{23} a_{11} a_{66} a_{77} a_{88}+a_{51} b_{32} a_{23} a_{75} a_{16} a_{67}+a_{51} b_{32} a_{23} a_{65} a_{86} a_{18}-a_{51} b_{32} a_{44} a_{13} a_{25} a_{88}+a_{43} b_{32} a_{55} a_{24} a_{66} a_{77}-a_{51} b_{32} a_{23} a_{85} a_{18} a_{66} \\
& -a_{51} b_{32} a_{23} a_{85} a_{18} a_{77}+b_{32} a_{21} a_{13} a_{66} a_{77} a_{88}+b_{32} a_{61} a_{23} a_{16} a_{77} a_{88}+a_{43} b_{32} a_{55} a_{24} a_{77} a_{88}-a_{51} a_{43} b_{32} a_{24} a_{15} a_{77}-b_{32} a_{44} a_{23} a_{11} a_{66} a_{88} \\
& +b_{32} a_{44} a_{81} a_{23} a_{18} a_{77}+a_{43} b_{32} a_{24} a_{66} a_{77} a_{88}-a_{43} b_{32} a_{81} a_{24} a_{18} a_{66}-a_{51} b_{32} a_{23} a_{15} a_{76} a_{67}-a_{51} b_{32} a_{23} a_{65} a_{16} a_{77}-a_{51} b_{32} a_{23} a_{65} a_{16} a_{88} \\
& -b_{32} a_{61} a_{23} a_{86} a_{18} a_{77}+b_{32} a_{23} a_{11} a_{76} a_{67} a_{88}+a_{43} b_{32} a_{24} a_{11} a_{77} a_{88}+b_{32} a_{55} a_{81} a_{23} a_{18} a_{66}-b_{32} a_{55} a_{23} a_{86} a_{67} a_{78}+b_{32} a_{44} a_{55} a_{61} a_{23} a_{16} \\
& -b_{32} a_{44} a_{21} a_{13} a_{76} a_{67}-b_{32} a_{44} a_{55} a_{23} a_{11} a_{77}-b_{32} a_{55} a_{23} a_{11} a_{77} a_{88} \text {, }
\end{aligned}
$$

$B_{6}=-a_{43} b_{32} a_{61} a_{24} a_{86} a_{18} a_{77}+a_{43} b_{32} a_{61} a_{24} a_{16} a_{77} a_{88}+a_{43} b_{32} a_{21} a_{84} a_{76} a_{18} a_{67}+a_{43} b_{32} a_{81} a_{24} a_{16} a_{67} a_{78}+a_{43} b_{32} a_{81} a_{24} a_{18} a_{66} a_{77}$ $-a_{43} b_{32} a_{81} a_{24} a_{76} a_{18} a_{67}+b_{32} a_{44} a_{55} a_{23} a_{66} a_{77} a_{88}+b_{32} a_{44} a_{55} a_{61} a_{23} a_{86} a_{18}+b_{32} a_{44} a_{55} a_{23} a_{11} a_{66} a_{88}+a_{51} b_{32} a_{23} a_{65} a_{16} a_{77} a_{88}$ $-a_{51} b_{32} a_{23} a_{65} a_{86} a_{18} a_{77}+a_{51} b_{32} a_{23} a_{75} a_{86} a_{18} a_{67}+a_{51} b_{32} a_{23} a_{85} a_{16} a_{67} a_{78}-a_{51} b_{32} a_{23} a_{75} a_{16} a_{67} a_{88}+a_{51} b_{32} a_{23} a_{85} a_{18} a_{66} a_{77}$ $-a_{51} b_{32} a_{23} a_{85} a_{76} a_{18} a_{67}+a_{43} b_{32} a_{55} a_{61} a_{24} a_{16} a_{77}-a_{43} b_{32} a_{55} a_{61} a_{24} a_{86} a_{18}+a_{43} b_{32} a_{55} a_{81} a_{24} a_{18} a_{77}+a_{43} b_{32} a_{55} a_{81} a_{24} a_{18} a_{66}$ $-a_{43} b_{32} a_{55} a_{24} a_{66} a_{77} a_{88}+a_{43} b_{32} a_{55} a_{24} a_{76} a_{67} a_{88}-a_{43} b_{32} a_{55} a_{24} a_{86} a_{67} a_{78}+a_{43} b_{32} a_{55} a_{21} a_{64} a_{86} a_{18}+a_{43} b_{32} a_{55} a_{21} a_{74} a_{16} a_{67}$ 
$+a_{51} a_{43} b_{32} a_{24} a_{65} a_{86} a_{18}+a_{51} a_{43} b_{32} a_{24} a_{75} a_{16} a_{67}+a_{51} b_{32} a_{44} a_{13} a_{25} a_{77} a_{88}+a_{51} b_{32} a_{44} a_{13} a_{25} a_{66} a_{88}+a_{51} b_{32} a_{44} a_{13} a_{25} a_{66} a_{77}$ $-a_{51} b_{32} a_{44} a_{23} a_{15} a_{77} a_{88}-a_{51} b_{32} a_{44} a_{13} a_{25} a_{76} a_{67}-a_{43} b_{32} a_{55} a_{24} a_{11} a_{66} a_{88}-a_{43} b_{32} a_{55} a_{24} a_{11} a_{66} a_{77}+a_{43} b_{32} a_{55} a_{24} a_{11} a_{76} a_{67}$ $+a_{43} b_{32} a_{55} a_{21} a_{14} a_{77} a_{88}-a_{51} b_{32} a_{44} a_{23} a_{15} a_{66} a_{88}-a_{51} b_{32} a_{44} a_{23} a_{15} a_{66} a_{77}+a_{51} b_{32} a_{44} a_{23} a_{15} a_{76} a_{67}+a_{51} b_{32} a_{44} a_{23} a_{65} a_{16} a_{88}$ $+a_{51} b_{32} a_{44} a_{23} a_{65} a_{16} a_{77}-a_{51} b_{32} a_{44} a_{23} a_{65} a_{86} a_{18}-a_{51} b_{32} a_{44} a_{23} a_{75} a_{16} a_{67}+a_{51} b_{32} a_{44} a_{23} a_{85} a_{18} a_{77}+a_{51} a_{43} b_{32} a_{64} a_{25} a_{16} a_{77}$ $+a_{51} b_{32} a_{44} a_{23} a_{85} a_{18} a_{66}+a_{51} a_{43} b_{32} a_{64} a_{25} a_{16} a_{88}+a_{51} b_{32} a_{13} a_{25} a_{66} a_{77} a_{88}-a_{51} a_{43} b_{32} a_{24} a_{15} a_{76} a_{67}-a_{51} b_{32} a_{13} a_{25} a_{76} a_{67} a_{88}$ $+a_{51} b_{32} a_{13} a_{25} a_{86} a_{67} a_{78}-a_{51} b_{32} a_{23} a_{15} a_{66} a_{77} a_{88}-a_{43} b_{32} a_{21} a_{84} a_{16} a_{67} a_{78}+a_{51} b_{32} a_{23} a_{15} a_{76} a_{67} a_{88}-a_{51} b_{32} a_{23} a_{15} a_{86} a_{67} a_{78}$ $-a_{51} a_{43} b_{32} a_{24} a_{65} a_{16} a_{77}-a_{51} a_{43} b_{32} a_{24} a_{65} a_{16} a_{88}+b_{32} a_{55} a_{61} a_{23} a_{86} a_{18} a_{77}+b_{32} a_{55} a_{81} a_{23} a_{76} a_{18} a_{67}-b_{32} a_{55} a_{81} a_{23} a_{16} a_{67} a_{78}$ $-b_{32} a_{55} a_{21} a_{13} a_{86} a_{67} a_{78}+b_{32} a_{55} a_{23} a_{11} a_{66} a_{77} a_{88}-b_{32} a_{55} a_{61} a_{23} a_{16} a_{77} a_{88}+b_{32} a_{55} a_{23} a_{11} a_{86} a_{67} a_{78}+b_{32} a_{55} a_{21} a_{13} a_{76} a_{67} a_{88}$ $-b_{32} a_{55} a_{21} a_{13} a_{66} a_{77} a_{88}-b_{32} a_{55} a_{23} a_{11} a_{76} a_{67} a_{88}-b_{32} a_{55} a_{81} a_{23} a_{18} a_{66} a_{77}-b_{32} a_{44} a_{21} a_{13} a_{86} a_{67} a_{78}+a_{51} a_{43} b_{32} a_{14} a_{25} a_{76} a_{67}$ $+a_{51} a_{43} b_{32} a_{24} a_{15} a_{77} a_{88}+a_{51} a_{43} b_{32} a_{24} a_{15} a_{66} a_{88}+a_{51} a_{43} b_{32} a_{24} a_{15} a_{66} a_{77}-a_{51} a_{43} b_{32} a_{64} a_{25} a_{86} a_{18}-a_{51} a_{43} b_{32} a_{74} a_{25} a_{16} a_{67}$ $+a_{51} a_{43} b_{32} a_{84} a_{25} a_{18} a_{77}+a_{51} a_{43} b_{32} a_{84} a_{25} a_{18} a_{66}-b_{32} a_{44} a_{55} a_{23} a_{76} a_{67} a_{88}-b_{32} a_{44} a_{55} a_{21} a_{13} a_{77} a_{88}+b_{32} a_{44} a_{55} a_{23} a_{86} a_{67} a_{78}$ $+b_{32} a_{44} a_{55} a_{21} a_{13} a_{76} a_{67}-b_{32} a_{44} a_{55} a_{81} a_{23} a_{18} a_{66}+b_{32} a_{44} a_{55} a_{23} a_{11} a_{66} a_{77}-b_{32} a_{44} a_{55} a_{61} a_{23} a_{16} a_{77}+b_{32} a_{44} a_{55} a_{23} a_{11} a_{77} a_{88}$ $-b_{32} a_{44} a_{55} a_{61} a_{23} a_{16} a_{88}-b_{32} a_{44} a_{55} a_{21} a_{13} a_{66} a_{88}-b_{32} a_{44} a_{55} a_{21} a_{13} a_{66} a_{77}+a_{43} b_{32} a_{55} a_{21} a_{14} a_{66} a_{77}-a_{43} b_{32} a_{55} a_{21} a_{14} a_{76} a_{67}$ $-a_{43} b_{32} a_{55} a_{21} a_{64} a_{16} a_{88}-a_{43} b_{32} a_{55} a_{21} a_{64} a_{16} a_{77}-a_{43} b_{32} a_{21} a_{84} a_{18} a_{66} a_{77}-b_{32} a_{44} a_{81} a_{23} a_{16} a_{67} a_{78}+a_{43} b_{32} a_{55} a_{21} a_{14} a_{66} a_{88}$ $+a_{43} b_{32} a_{21} a_{14} a_{66} a_{77} a_{88}-a_{43} b_{32} a_{21} a_{14} a_{76} a_{67} a_{88}+a_{43} b_{32} a_{21} a_{14} a_{86} a_{67} a_{78}-a_{43} b_{32} a_{21} a_{64} a_{16} a_{77} a_{88}+a_{43} b_{32} a_{21} a_{64} a_{86} a_{18} a_{77}$ $+a_{43} b_{32} a_{21} a_{74} a_{16} a_{67} a_{88}-a_{43} b_{32} a_{21} a_{74} a_{86} a_{18} a_{67}-a_{43} b_{32} a_{55} a_{21} a_{84} a_{18} a_{77}-a_{43} b_{32} a_{55} a_{21} a_{84} a_{18} a_{66}+a_{43} b_{32} a_{55} a_{61} a_{24} a_{16} a_{88}$ $-a_{43} b_{32} a_{24} a_{11} a_{66} a_{77} a_{88}+a_{43} b_{32} a_{24} a_{11} a_{76} a_{67} a_{88}-a_{51} a_{43} b_{32} a_{14} a_{25} a_{66} a_{77}+b_{32} a_{44} a_{81} a_{23} a_{76} a_{18} a_{67}-a_{51} a_{43} b_{32} a_{24} a_{85} a_{18} a_{77}$ $-a_{51} a_{43} b_{32} a_{24} a_{85} a_{18} a_{66}-a_{51} a_{43} b_{32} a_{14} a_{25} a_{77} a_{88}-a_{51} a_{43} b_{32} a_{14} a_{25} a_{66} a_{88}-a_{43} b_{32} a_{55} a_{24} a_{11} a_{77} a_{88}+b_{32} a_{44} a_{61} a_{23} a_{86} a_{18} a_{77}$ $+b_{32} a_{44} a_{23} a_{11} a_{66} a_{77} a_{88}+b_{32} a_{44} a_{21} a_{13} a_{76} a_{67} a_{88}+b_{32} a_{44} a_{23} a_{11} a_{86} a_{67} a_{78}-b_{32} a_{44} a_{81} a_{23} a_{18} a_{66} a_{77}-b_{32} a_{44} a_{61} a_{23} a_{16} a_{77} a_{88}$ $-b_{32} a_{44} a_{55} a_{81} a_{23} a_{18} a_{77}-b_{32} a_{44} a_{55} a_{23} a_{11} a_{76} a_{67}-b_{32} a_{44} a_{21} a_{13} a_{66} a_{77} a_{88}-b_{32} a_{44} a_{23} a_{11} a_{76} a_{67} a_{88}-a_{43} b_{32} a_{24} a_{11} a_{86} a_{67} a_{78}$,

$B_{7}=-a_{43} b_{32} a_{55} a_{81} a_{24} a_{18} a_{66} a_{77}+a_{43} b_{32} a_{55} a_{81} a_{24} a_{76} a_{18} a_{67}+a_{43} b_{32} a_{55} a_{61} a_{24} a_{86} a_{18} a_{77}+a_{43} b_{32} a_{55} a_{24} a_{11} a_{86} a_{67} a_{78}$ $-a_{43} b_{32} a_{55} a_{24} a_{11} a_{76} a_{67} a_{88}-a_{43} b_{32} a_{55} a_{21} a_{14} a_{66} a_{77} a_{88}+a_{43} b_{32} a_{55} a_{24} a_{11} a_{66} a_{77} a_{88}+a_{43} b_{32} a_{55} a_{21} a_{84} a_{16} a_{67} a_{78}$ $+a_{43} b_{32} a_{55} a_{21} a_{84} a_{18} a_{66} a_{77}-a_{43} b_{32} a_{55} a_{21} a_{84} a_{76} a_{18} a_{67}-a_{43} b_{32} a_{55} a_{61} a_{24} a_{16} a_{77} a_{88}+a_{51} b_{32} a_{44} a_{13} a_{25} a_{76} a_{67} a_{88}$ $-a_{51} b_{32} a_{44} a_{13} a_{25} a_{86} a_{67} a_{78}-b_{32} a_{44} a_{55} a_{61} a_{23} a_{86} a_{18} a_{77}-a_{51} b_{32} a_{44} a_{13} a_{25} a_{66} a_{77} a_{88}-a_{43} b_{32} a_{55} a_{21} a_{64} a_{86} a_{18} a_{77}$ $-a_{43} b_{32} a_{55} a_{21} a_{74} a_{16} a_{67} a_{88}-a_{43} b_{32} a_{55} a_{21} a_{14} a_{86} a_{67} a_{78}+a_{43} b_{32} a_{55} a_{21} a_{64} a_{16} a_{77} a_{88}+a_{51} a_{43} b_{32} a_{14} a_{25} a_{66} a_{77} a_{88}$ $+a_{43} b_{32} a_{55} a_{21} a_{14} a_{76} a_{67} a_{88}+a_{51} a_{43} b_{32} a_{24} a_{85} a_{16} a_{67} a_{78}+a_{51} a_{43} b_{32} a_{24} a_{85} a_{18} a_{66} a_{77}-a_{51} a_{43} b_{32} a_{24} a_{75} a_{16} a_{67} a_{88}$ $+a_{51} a_{43} b_{32} a_{24} a_{75} a_{86} a_{18} a_{67}-a_{51} a_{43} b_{32} a_{24} a_{65} a_{86} a_{18} a_{77}+a_{43} b_{32} a_{55} a_{21} a_{74} a_{86} a_{18} a_{67}+a_{51} a_{43} b_{32} a_{24} a_{65} a_{16} a_{77} a_{88}$ $+a_{51} b_{32} a_{44} a_{23} a_{75} a_{16} a_{67} a_{88}-a_{51} b_{32} a_{44} a_{23} a_{75} a_{86} a_{18} a_{67}-a_{51} b_{32} a_{44} a_{23} a_{65} a_{16} a_{77} a_{88}+a_{51} b_{32} a_{44} a_{23} a_{65} a_{86} a_{18} a_{77}$ $+a_{51} b_{32} a_{44} a_{23} a_{15} a_{86} a_{67} a_{78}+a_{51} b_{32} a_{44} a_{23} a_{15} a_{66} a_{77} a_{88}-a_{51} b_{32} a_{44} a_{23} a_{15} a_{76} a_{67} a_{88}-b_{32} a_{44} a_{55} a_{23} a_{11} a_{66} a_{77} a_{88}$ $+b_{32} a_{44} a_{55} a_{21} a_{13} a_{86} a_{67} a_{78}-b_{32} a_{44} a_{55} a_{21} a_{13} a_{76} a_{67} a_{88}-b_{32} a_{44} a_{55} a_{81} a_{23} a_{76} a_{18} a_{67}+b_{32} a_{44} a_{55} a_{81} a_{23} a_{16} a_{67} a_{78}$ $+a_{51} a_{43} b_{32} a_{84} a_{25} a_{76} a_{18} a_{67}-a_{51} a_{43} b_{32} a_{84} a_{25} a_{16} a_{67} a_{78}-a_{51} a_{43} b_{32} a_{84} a_{25} a_{18} a_{66} a_{77}+a_{51} a_{43} b_{32} a_{74} a_{25} a_{16} a_{67} a_{88}$ $-a_{51} a_{43} b_{32} a_{74} a_{25} a_{86} a_{18} a_{67}-a_{51} a_{43} b_{32} a_{64} a_{25} a_{16} a_{77} a_{88}+a_{51} a_{43} b_{32} a_{64} a_{25} a_{86} a_{18} a_{77}+a_{51} a_{43} b_{32} a_{14} a_{25} a_{86} a_{67} a_{78}$ $-a_{51} a_{43} b_{32} a_{24} a_{15} a_{66} a_{77} a_{88}-a_{51} a_{43} b_{32} a_{24} a_{15} a_{86} a_{67} a_{78}-a_{51} a_{43} b_{32} a_{14} a_{25} a_{76} a_{67} a_{88}+a_{51} a_{43} b_{32} a_{24} a_{15} a_{76} a_{67} a_{88}$ $-a_{51} a_{43} b_{32} a_{24} a_{85} a_{76} a_{18} a_{67}-a_{43} b_{32} a_{55} a_{81} a_{24} a_{16} a_{67} a_{78}+a_{51} b_{32} a_{44} a_{23} a_{85} a_{76} a_{18} a_{67}-a_{51} b_{32} a_{44} a_{23} a_{85} a_{16} a_{67} a_{78}$ $-a_{51} b_{32} a_{44} a_{23} a_{85} a_{18} a_{66} a_{77}+b_{32} a_{44} a_{55} a_{81} a_{23} a_{18} a_{66} a_{77}+b_{32} a_{44} a_{55} a_{21} a_{13} a_{66} a_{77} a_{88}+b_{32} a_{44} a_{55} a_{61} a_{23} a_{16} a_{77} a_{88}$ $+b_{32} a_{44} a_{55} a_{23} a_{11} a_{76} a_{67} a_{88}-b_{32} a_{44} a_{55} a_{23} a_{11} a_{86} a_{67} a_{78}$

$$
C_{1}=-b_{42} a_{24} \text {, }
$$

$$
C_{2}=b_{42} a_{24} a_{66}+b_{42} a_{24} a_{88}+b_{42} a_{55} a_{24}+b_{42} a_{24} a_{77}+b_{42} a_{24} a_{11}+b_{42} a_{33} a_{24}-b_{42} a_{21} a_{14}
$$

$C_{3}=b_{42} a_{21} a_{14} a_{77}+b_{42} a_{81} a_{24} a_{18}-b_{42} a_{24} a_{66} a_{77}-b_{42} a_{21} a_{64} a_{16}+b_{42} a_{21} a_{14} a_{66}-b_{42} a_{24} a_{77} a_{88}+b_{42} a_{24} a_{76} a_{67}-b_{42} a_{24} a_{11} a_{66}$ $-b_{42} a_{21} a_{84} a_{18}+b_{42} a_{61} a_{24} a_{16}+b_{42} a_{21} a_{14} a_{88}-a_{51} b_{42} a_{14} a_{25}+a_{51} b_{42} a_{24} a_{15}-b_{42} a_{24} a_{11} a_{88}-b_{42} a_{24} a_{11} a_{77}-b_{42} a_{24} a_{66} a_{88}$ 


$$
\begin{aligned}
& -b_{42} a_{33} a_{24} a_{77}-b_{42} a_{33} a_{24} a_{88}-b_{42} a_{33} a_{24} a_{11}+b_{42} a_{33} a_{21} a_{14}-b_{42} a_{33} a_{55} a_{24}-b_{42} a_{55} a_{24} a_{66}-b_{42} a_{55} a_{24} a_{77}-b_{42} a_{55} a_{24} a_{88} \\
& -b_{42} a_{55} a_{24} a_{11}+b_{42} a_{55} a_{21} a_{14}-b_{42} a_{33} a_{24} a_{66} \text {, } \\
& C_{4}=b_{42} a_{33} a_{55} a_{24} a_{88}-b_{42} a_{55} a_{81} a_{24} a_{18}+b_{42} a_{55} a_{24} a_{77} a_{88}+b_{42} a_{55} a_{24} a_{66} a_{88}-b_{42} a_{33} a_{21} a_{14} a_{88}-b_{42} a_{33} a_{21} a_{14} a_{77} \\
& -b_{42} a_{21} a_{74} a_{16} a_{67}+b_{42} a_{55} a_{24} a_{11} a_{66}-b_{42} a_{55} a_{21} a_{14} a_{88}-a_{51} b_{42} a_{24} a_{15} a_{77}-b_{42} a_{55} a_{21} a_{14} a_{77}+a_{51} b_{42} a_{14} a_{25} a_{77} \\
& +a_{51} b_{42} a_{14} a_{25} a_{66}-a_{51} b_{42} a_{24} a_{15} a_{88}-a_{51} b_{42} a_{33} a_{24} a_{15}+a_{51} b_{42} a_{14} a_{25} a_{88}-b_{42} a_{33} a_{81} a_{24} a_{18}+b_{42} a_{33} a_{24} a_{11} a_{77} \\
& -b_{42} a_{21} a_{14} a_{77} a_{88}-b_{42} a_{21} a_{14} a_{66} a_{88}-b_{42} a_{21} a_{14} a_{66} a_{77}+b_{42} a_{24} a_{11} a_{66} a_{88}+b_{42} a_{24} a_{11} a_{66} a_{77}-b_{42} a_{24} a_{11} a_{76} a_{67} \\
& -b_{42} a_{33} a_{61} a_{24} a_{16}+b_{42} a_{21} a_{84} a_{18} a_{77}+b_{42} a_{21} a_{84} a_{18} a_{66}-b_{42} a_{61} a_{24} a_{16} a_{88}+b_{42} a_{21} a_{14} a_{76} a_{67}+b_{42} a_{21} a_{64} a_{16} a_{88} \\
& -b_{42} a_{24} a_{76} a_{67} a_{88}+b_{42} a_{24} a_{86} a_{67} a_{78}+b_{42} a_{24} a_{11} a_{77} a_{88}+b_{42} a_{33} a_{21} a_{64} a_{16}+b_{42} a_{33} a_{55} a_{24} a_{11}+b_{42} a_{33} a_{24} a_{66} a_{88} \\
& +b_{42} a_{33} a_{55} a_{24} a_{77}+b_{42} a_{33} a_{55} a_{24} a_{66}-b_{42} a_{21} a_{64} a_{86} a_{18}-b_{42} a_{33} a_{21} a_{14} a_{66}+b_{42} a_{33} a_{21} a_{84} a_{18}-b_{42} a_{55} a_{21} a_{14} a_{66} \\
& +b_{42} a_{55} a_{21} a_{64} a_{16}+b_{42} a_{55} a_{21} a_{84} a_{18}-b_{42} a_{55} a_{61} a_{24} a_{16}+b_{42} a_{21} a_{64} a_{16} a_{77}+a_{51} b_{42} a_{24} a_{65} a_{16}+a_{51} b_{42} a_{24} a_{85} a_{18} \\
& -a_{51} b_{42} a_{64} a_{25} a_{16}-a_{51} b_{42} a_{84} a_{25} a_{18}+a_{51} b_{42} a_{33} a_{14} a_{25}+b_{42} a_{61} a_{24} a_{86} a_{18}-b_{42} a_{81} a_{24} a_{18} a_{77}-b_{42} a_{81} a_{24} a_{18} a_{66} \\
& -b_{42} a_{61} a_{24} a_{16} a_{77}+b_{42} a_{33} a_{24} a_{77} a_{88}-b_{42} a_{33} a_{55} a_{21} a_{14}+b_{42} a_{33} a_{24} a_{11} a_{88}+b_{42} a_{33} a_{24} a_{66} a_{77}-b_{42} a_{33} a_{24} a_{76} a_{67} \\
& +b_{42} a_{24} a_{66} a_{77} a_{88}+b_{42} a_{55} a_{24} a_{11} a_{77}+b_{42} a_{33} a_{24} a_{11} a_{66}+b_{42} a_{55} a_{24} a_{66} a_{77}-b_{42} a_{55} a_{24} a_{76} a_{67}+b_{42} a_{55} a_{24} a_{11} a_{88} \\
& -a_{51} b_{42} a_{24} a_{15} a_{66} \text {, }
\end{aligned}
$$

$C_{5}=-b_{42} a_{61} a_{24} a_{86} a_{18} a_{77}+b_{42} a_{61} a_{24} a_{16} a_{77} a_{88}+b_{42} a_{21} a_{84} a_{76} a_{18} a_{67}+b_{42} a_{81} a_{24} a_{16} a_{67} a_{78}-b_{42} a_{21} a_{64} a_{16} a_{77} a_{88}$ $+b_{42} a_{21} a_{14} a_{86} a_{67} a_{78}+b_{42} a_{21} a_{64} a_{86} a_{18} a_{77}-b_{42} a_{21} a_{74} a_{86} a_{18} a_{67}+b_{42} a_{21} a_{74} a_{16} a_{67} a_{88}-b_{42} a_{55} a_{21} a_{84} a_{18} a_{66}$ $-b_{42} a_{55} a_{21} a_{84} a_{18} a_{77}+b_{42} a_{55} a_{61} a_{24} a_{16} a_{88}-b_{42} a_{21} a_{84} a_{18} a_{66} a_{77}-b_{42} a_{21} a_{84} a_{16} a_{67} a_{78}-b_{42} a_{24} a_{11} a_{86} a_{67} a_{78}$ $+b_{42} a_{24} a_{11} a_{76} a_{67} a_{88}-b_{42} a_{24} a_{11} a_{66} a_{77} a_{88}-b_{42} a_{21} a_{14} a_{76} a_{67} a_{88}+b_{42} a_{21} a_{14} a_{66} a_{77} a_{88}+b_{42} a_{55} a_{61} a_{24} a_{16} a_{77}$ $-b_{42} a_{55} a_{61} a_{24} a_{86} a_{18}-b_{42} a_{55} a_{24} a_{86} a_{67} a_{78}+b_{42} a_{55} a_{24} a_{76} a_{67} a_{88}-b_{42} a_{55} a_{24} a_{66} a_{77} a_{88}+b_{42} a_{55} a_{81} a_{24} a_{18} a_{66}$ $-b_{42} a_{55} a_{24} a_{11} a_{77} a_{88}+b_{42} a_{55} a_{81} a_{24} a_{18} a_{77}-b_{42} a_{55} a_{21} a_{64} a_{16} a_{77}-b_{42} a_{55} a_{24} a_{11} a_{66} a_{88}-b_{42} a_{55} a_{24} a_{11} a_{66} a_{77}$ $+b_{42} a_{55} a_{24} a_{11} a_{76} a_{67}+b_{42} a_{55} a_{21} a_{14} a_{77} a_{88}+b_{42} a_{55} a_{21} a_{14} a_{66} a_{88}+b_{42} a_{55} a_{21} a_{14} a_{66} a_{77}-b_{42} a_{55} a_{21} a_{64} a_{16} a_{88}$ $-b_{42} a_{55} a_{21} a_{14} a_{76} a_{67}+a_{51} b_{42} a_{24} a_{15} a_{77} a_{88}+b_{42} a_{55} a_{21} a_{64} a_{86} a_{18}+b_{42} a_{55} a_{21} a_{74} a_{16} a_{67}-b_{42} a_{33} a_{21} a_{84} a_{18} a_{77}$ $+b_{42} a_{33} a_{61} a_{24} a_{16} a_{88}-b_{42} a_{33} a_{21} a_{84} a_{18} a_{66}+b_{42} a_{33} a_{81} a_{24} a_{18} a_{77}-b_{42} a_{33} a_{61} a_{24} a_{86} a_{18}+b_{42} a_{33} a_{61} a_{24} a_{16} a_{77}$ $-a_{51} b_{42} a_{33} a_{14} a_{25} a_{66}+b_{42} a_{33} a_{81} a_{24} a_{18} a_{66}-b_{42} a_{33} a_{24} a_{11} a_{66} a_{88}-b_{42} a_{33} a_{24} a_{11} a_{77} a_{88}-b_{42} a_{33} a_{24} a_{86} a_{67} a_{78}$ $+b_{42} a_{33} a_{24} a_{76} a_{67} a_{88}-b_{42} a_{33} a_{24} a_{66} a_{77} a_{88}-a_{51} b_{42} a_{14} a_{25} a_{66} a_{77}+a_{51} b_{42} a_{14} a_{25} a_{76} a_{67}+a_{51} b_{42} a_{84} a_{25} a_{18} a_{77}$ $-a_{51} b_{42} a_{14} a_{25} a_{77} a_{88}-a_{51} b_{42} a_{14} a_{25} a_{66} a_{88}+a_{51} b_{42} a_{84} a_{25} a_{18} a_{66}-a_{51} b_{42} a_{33} a_{14} a_{25} a_{88}-b_{42} a_{33} a_{24} a_{11} a_{66} a_{77}$ $-a_{51} b_{42} a_{74} a_{25} a_{16} a_{67}+b_{42} a_{33} a_{24} a_{11} a_{76} a_{67}-a_{51} b_{42} a_{24} a_{15} a_{76} a_{67}-a_{51} b_{42} a_{24} a_{65} a_{16} a_{88}-a_{51} b_{42} a_{24} a_{65} a_{16} a_{77}$ $+a_{51} b_{42} a_{24} a_{65} a_{86} a_{18}+a_{51} b_{42} a_{24} a_{75} a_{16} a_{67}+a_{51} b_{42} a_{64} a_{25} a_{16} a_{88}+a_{51} b_{42} a_{64} a_{25} a_{16} a_{77}-a_{51} b_{42} a_{64} a_{25} a_{86} a_{18}$ $+a_{51} b_{42} a_{24} a_{15} a_{66} a_{77}+b_{42} a_{33} a_{21} a_{14} a_{66} a_{77}+b_{42} a_{33} a_{21} a_{14} a_{66} a_{88}+b_{42} a_{33} a_{21} a_{14} a_{77} a_{88}-b_{42} a_{33} a_{21} a_{64} a_{16} a_{77}$ $+b_{42} a_{33} a_{21} a_{64} a_{86} a_{18}-b_{42} a_{33} a_{21} a_{64} a_{16} a_{88}-b_{42} a_{33} a_{21} a_{14} a_{76} a_{67}+b_{42} a_{33} a_{55} a_{61} a_{24} a_{16}-b_{42} a_{33} a_{55} a_{21} a_{84} a_{18}$ $-b_{42} a_{33} a_{55} a_{21} a_{64} a_{16}+b_{42} a_{33} a_{55} a_{21} a_{14} a_{66}+b_{42} a_{33} a_{55} a_{21} a_{14} a_{77}+b_{42} a_{33} a_{55} a_{21} a_{14} a_{88}-b_{42} a_{33} a_{55} a_{24} a_{11} a_{66}$ $+b_{42} a_{33} a_{21} a_{74} a_{16} a_{67}+b_{42} a_{33} a_{55} a_{81} a_{24} a_{18}-b_{42} a_{33} a_{55} a_{24} a_{77} a_{88}-b_{42} a_{33} a_{55} a_{24} a_{66} a_{88}+a_{51} b_{42} a_{24} a_{15} a_{66} a_{88}$ $-b_{42} a_{33} a_{55} a_{24} a_{66} a_{77}+b_{42} a_{33} a_{55} a_{24} a_{76} a_{67}-b_{42} a_{33} a_{55} a_{24} a_{11} a_{77}-b_{42} a_{33} a_{55} a_{24} a_{11} a_{88}-a_{51} b_{42} a_{24} a_{85} a_{18} a_{66}$ $-a_{51} b_{42} a_{33} a_{24} a_{65} a_{16}-a_{51} b_{42} a_{24} a_{85} a_{18} a_{77}+a_{51} b_{42} a_{33} a_{24} a_{15} a_{88}+a_{51} b_{42} a_{33} a_{64} a_{25} a_{16}+a_{51} b_{42} a_{33} a_{24} a_{15} a_{66}$ $-a_{51} b_{42} a_{33} a_{14} a_{25} a_{77}-b_{42} a_{81} a_{24} a_{76} a_{18} a_{67}+a_{51} b_{42} a_{33} a_{24} a_{15} a_{77}+b_{42} a_{81} a_{24} a_{18} a_{66} a_{77}+a_{51} b_{42} a_{33} a_{84} a_{25} a_{18}$ $-a_{51} b_{42} a_{33} a_{24} a_{85} a_{18}$

$C_{6}=-a_{51} b_{42} a_{14} a_{25} a_{76} a_{67} a_{88}-a_{51} b_{42} a_{24} a_{75} a_{16} a_{67} a_{88}+a_{51} b_{42} a_{14} a_{25} a_{86} a_{67} a_{78}-a_{51} b_{42} a_{24} a_{15} a_{66} a_{77} a_{88}-a_{51} b_{42} a_{24} a_{15} a_{86} a_{67} a_{78}$ $+a_{51} b_{42} a_{24} a_{15} a_{76} a_{67} a_{88}+a_{51} b_{42} a_{24} a_{65} a_{16} a_{77} a_{88}+a_{51} b_{42} a_{24} a_{75} a_{86} a_{18} a_{67}+a_{51} b_{42} a_{24} a_{85} a_{16} a_{67} a_{78}+a_{51} b_{42} a_{24} a_{85} a_{18} a_{66} a_{77}$ $-a_{51} b_{42} a_{24} a_{85} a_{76} a_{18} a_{67}-a_{51} b_{42} a_{64} a_{25} a_{16} a_{77} a_{88}+a_{51} b_{42} a_{64} a_{25} a_{86} a_{18} a_{77}-a_{51} b_{42} a_{74} a_{25} a_{86} a_{18} a_{67}+a_{51} b_{42} a_{14} a_{25} a_{66} a_{77} a_{88}$ $-a_{51} b_{42} a_{84} a_{25} a_{16} a_{67} a_{78}-a_{51} b_{42} a_{84} a_{25} a_{18} a_{66} a_{77}+a_{51} b_{42} a_{74} a_{25} a_{16} a_{67} a_{88}-a_{51} b_{42} a_{33} a_{24} a_{65} a_{86} a_{18}+a_{51} b_{42} a_{84} a_{25} a_{76} a_{18} a_{67}$ $-a_{51} b_{42} a_{33} a_{24} a_{15} a_{77} a_{88}-a_{51} b_{42} a_{33} a_{24} a_{15} a_{66} a_{88}-a_{51} b_{42} a_{33} a_{24} a_{15} a_{66} a_{77}+a_{51} b_{42} a_{33} a_{24} a_{15} a_{76} a_{67}+a_{51} b_{42} a_{33} a_{24} a_{65} a_{16} a_{77}$ 
$+a_{51} b_{42} a_{33} a_{24} a_{65} a_{16} a_{88}-a_{51} b_{42} a_{33} a_{24} a_{75} a_{16} a_{67}+a_{51} b_{42} a_{33} a_{24} a_{85} a_{18} a_{77}+a_{51} b_{42} a_{33} a_{24} a_{85} a_{18} a_{66}-a_{51} b_{42} a_{33} a_{64} a_{25} a_{16} a_{88}$ $-a_{51} b_{42} a_{33} a_{64} a_{25} a_{16} a_{77}+a_{51} b_{42} a_{33} a_{64} a_{25} a_{86} a_{18}+a_{51} b_{42} a_{33} a_{74} a_{25} a_{16} a_{67}+a_{51} b_{42} a_{33} a_{14} a_{25} a_{77} a_{88}+a_{51} b_{42} a_{33} a_{14} a_{25} a_{66} a_{88}$ $+a_{51} b_{42} a_{33} a_{14} a_{25} a_{66} a_{77}-a_{51} b_{42} a_{33} a_{14} a_{25} a_{76} a_{67}-a_{51} b_{42} a_{33} a_{84} a_{25} a_{18} a_{77}-a_{51} b_{42} a_{33} a_{84} a_{25} a_{18} a_{66}+b_{42} a_{55} a_{21} a_{84} a_{16} a_{67} a_{78}$ $+b_{42} a_{55} a_{61} a_{24} a_{86} a_{18} a_{77}-b_{42} a_{55} a_{61} a_{24} a_{16} a_{77} a_{88}-b_{42} a_{55} a_{21} a_{84} a_{76} a_{18} a_{67}+b_{42} a_{55} a_{21} a_{84} a_{18} a_{66} a_{77}+b_{42} a_{33} a_{21} a_{84} a_{16} a_{67} a_{78}$ $+b_{42} a_{55} a_{21} a_{74} a_{86} a_{18} a_{67}-b_{42} a_{55} a_{21} a_{74} a_{16} a_{67} a_{88}-b_{42} a_{55} a_{21} a_{64} a_{86} a_{18} a_{77}+b_{42} a_{55} a_{21} a_{64} a_{16} a_{77} a_{88}-b_{42} a_{55} a_{21} a_{14} a_{86} a_{67} a_{78}$ $+b_{42} a_{55} a_{21} a_{14} a_{76} a_{67} a_{88}-b_{42} a_{55} a_{21} a_{14} a_{66} a_{77} a_{88}+b_{42} a_{55} a_{24} a_{11} a_{86} a_{67} a_{78}-b_{42} a_{55} a_{24} a_{11} a_{76} a_{67} a_{88}+b_{42} a_{55} a_{24} a_{11} a_{66} a_{77} a_{88}$ $+b_{42} a_{55} a_{81} a_{24} a_{76} a_{18} a_{67}-b_{42} a_{55} a_{81} a_{24} a_{18} a_{66} a_{77}+b_{42} a_{33} a_{21} a_{84} a_{18} a_{66} a_{77}-b_{42} a_{33} a_{21} a_{84} a_{76} a_{18} a_{67}-b_{42} a_{33} a_{21} a_{84} a_{76} a_{18} a_{67}$ $-b_{42} a_{55} a_{81} a_{24} a_{16} a_{67} a_{78}+b_{42} a_{33} a_{21} a_{64} a_{16} a_{77} a_{88}-b_{42} a_{33} a_{21} a_{14} a_{86} a_{67} a_{78}+b_{42} a_{33} a_{21} a_{14} a_{76} a_{67} a_{88}-b_{42} a_{33} a_{21} a_{14} a_{66} a_{77} a_{88}$ $+b_{42} a_{33} a_{24} a_{11} a_{86} a_{67} a_{78}-b_{42} a_{33} a_{24} a_{11} a_{76} a_{67} a_{88}+b_{42} a_{33} a_{24} a_{11} a_{66} a_{77} a_{88}+b_{42} a_{33} a_{81} a_{24} a_{76} a_{18} a_{67}-b_{42} a_{33} a_{81} a_{24} a_{18} a_{66} a_{77}$ $-b_{42} a_{33} a_{81} a_{24} a_{16} a_{67} a_{78}+b_{42} a_{33} a_{61} a_{24} a_{86} a_{18} a_{77}-b_{42} a_{33} a_{21} a_{64} a_{86} a_{18} a_{77}-b_{42} a_{33} a_{61} a_{24} a_{16} a_{77} a_{88}-b_{42} a_{33} a_{61} a_{24} a_{16} a_{77} a_{88}$ $+b_{42} a_{33} a_{21} a_{74} a_{86} a_{18} a_{67}-b_{42} a_{33} a_{21} a_{74} a_{16} a_{67} a_{88}-b_{42} a_{33} a_{55} a_{61} a_{24} a_{16} a_{77}-b_{42} a_{33} a_{55} a_{61} a_{24} a_{16} a_{88}+b_{42} a_{33} a_{55} a_{21} a_{84} a_{18} a_{66}$ $+b_{42} a_{33} a_{55} a_{21} a_{84} a_{18} a_{77}+b_{42} a_{33} a_{55} a_{61} a_{24} a_{86} a_{18}-b_{42} a_{33} a_{55} a_{81} a_{24} a_{18} a_{66}-b_{42} a_{33} a_{55} a_{81} a_{24} a_{18} a_{77}-b_{42} a_{33} a_{55} a_{24} a_{76} a_{67} a_{88}$ $+b_{42} a_{33} a_{55} a_{24} a_{66} a_{77} a_{88}+b_{42} a_{33} a_{55} a_{24} a_{11} a_{77} a_{88}+b_{42} a_{33} a_{55} a_{24} a_{86} a_{67} a_{78}+b_{42} a_{33} a_{55} a_{24} a_{11} a_{66} a_{77}+b_{42} a_{33} a_{55} a_{24} a_{11} a_{66} a_{88}$ $-b_{42} a_{33} a_{55} a_{24} a_{11} a_{76} a_{67}+b_{42} a_{33} a_{55} a_{21} a_{14} a_{76} a_{67}-b_{42} a_{33} a_{55} a_{21} a_{14} a_{66} a_{77}-b_{42} a_{33} a_{55} a_{21} a_{14} a_{66} a_{88}-b_{42} a_{33} a_{55} a_{21} a_{14} a_{77} a_{88}$ $+b_{42} a_{33} a_{55} a_{21} a_{64} a_{16} a_{77}+b_{42} a_{33} a_{55} a_{21} a_{64} a_{16} a_{88}-b_{42} a_{33} a_{55} a_{21} a_{74} a_{16} a_{67}-b_{42} a_{33} a_{55} a_{21} a_{64} a_{86} a_{18}-a_{51} b_{42} a_{24} a_{65} a_{86} a_{18} a_{77}$,

$C_{7}=-a_{51} b_{42} a_{33} a_{24} a_{15} a_{76} a_{67} a_{88}+a_{51} b_{42} a_{33} a_{24} a_{65} a_{86} a_{18} a_{77}+a_{51} b_{42} a_{33} a_{24} a_{75} a_{16} a_{67} a_{88}+a_{51} b_{42} a_{33} a_{24} a_{15} a_{86} a_{67} a_{78}$ $-a_{51} b_{42} a_{33} a_{24} a_{65} a_{16} a_{77} a_{88}-a_{51} b_{42} a_{33} a_{24} a_{85} a_{18} a_{66} a_{77}+a_{51} b_{42} a_{33} a_{24} a_{85} a_{76} a_{18} a_{67}-a_{51} b_{42} a_{33} a_{24} a_{75} a_{86} a_{18} a_{67}$ $-a_{51} b_{42} a_{33} a_{24} a_{85} a_{16} a_{67} a_{78}-a_{51} b_{42} a_{33} a_{74} a_{25} a_{16} a_{67} a_{88}+a_{51} b_{42} a_{33} a_{74} a_{25} a_{86} a_{18} a_{67}-a_{51} b_{42} a_{33} a_{14} a_{25} a_{66} a_{77} a_{88}$ $+a_{51} b_{42} a_{33} a_{84} a_{25} a_{16} a_{67} a_{78}+a_{51} b_{42} a_{33} a_{84} a_{25} a_{18} a_{66} a_{77}-a_{51} b_{42} a_{33} a_{84} a_{25} a_{76} a_{18} a_{67}-b_{42} a_{33} a_{55} a_{21} a_{84} a_{16} a_{67} a_{78}$ $+b_{42} a_{33} a_{55} a_{21} a_{84} a_{76} a_{18} a_{67}-b_{42} a_{33} a_{55} a_{21} a_{84} a_{18} a_{66} a_{77}+b_{42} a_{33} a_{55} a_{81} a_{24} a_{16} a_{67} a_{78}-b_{42} a_{33} a_{55} a_{61} a_{24} a_{86} a_{18} a_{77}$ $+a_{51} b_{42} a_{33} a_{64} a_{25} a_{16} a_{77} a_{88}-a_{51} b_{42} a_{33} a_{64} a_{25} a_{86} a_{18} a_{77}-b_{42} a_{33} a_{55} a_{81} a_{24} a_{76} a_{18} a_{67}-b_{42} a_{33} a_{55} a_{24} a_{11} a_{66} a_{77} a_{88}$ $+b_{42} a_{33} a_{55} a_{24} a_{11} a_{76} a_{67} a_{88}-b_{42} a_{33} a_{55} a_{24} a_{11} a_{86} a_{67} a_{78}+b_{42} a_{33} a_{55} a_{21} a_{14} a_{66} a_{77} a_{88}-b_{42} a_{33} a_{55} a_{21} a_{14} a_{76} a_{67} a_{88}$ $+b_{42} a_{33} a_{55} a_{21} a_{14} a_{86} a_{67} a_{78}-b_{42} a_{33} a_{55} a_{21} a_{64} a_{16} a_{77} a_{88}+b_{42} a_{33} a_{55} a_{21} a_{64} a_{86} a_{18} a_{77}+b_{42} a_{33} a_{55} a_{21} a_{74} a_{16} a_{67} a_{88}$ $-b_{42} a_{33} a_{55} a_{21} a_{74} a_{86} a_{18} a_{67}+b_{42} a_{33} a_{55} a_{61} a_{24} a_{16} a_{77} a_{88}+b_{42} a_{33} a_{55} a_{81} a_{24} a_{18} a_{66} a_{77}+a_{51} b_{42} a_{33} a_{14} a_{25} a_{76} a_{67} a_{88}$ $-a_{51} b_{42} a_{33} a_{14} a_{25} a_{86} a_{67} a_{78}+a_{51} b_{42} a_{33} a_{24} a_{15} a_{66} a_{77} a_{88}$,

$$
E_{1}=-b_{32} a_{25},
$$

$$
E_{2}=b_{32} a_{25} a_{77}+b_{32} a_{33} a_{25}-b_{32} a_{21} a_{15}+b_{32} a_{44} a_{25}+b_{32} a_{25} a_{88}+b_{32} a_{25} a_{11}+b_{32} a_{25} a_{66} \text {, }
$$

$E_{3}=b_{32} a_{21} a_{15} a_{88}+b_{32} a_{21} a_{15} a_{77}+b_{32} a_{21} a_{15} a_{66}-b_{32} a_{21} a_{65} a_{16}-b_{32} a_{21} a_{85} a_{18}+b_{32} a_{25} a_{76} a_{67}-b_{32} a_{25} a_{11} a_{88}-b_{32} a_{25} a_{11} a_{77}$ $+b_{32} a_{61} a_{25} a_{16}+b_{32} a_{81} a_{25} a_{18}-b_{32} a_{25} a_{11} a_{66}-b_{32} a_{44} a_{25} a_{77}-b_{32} a_{44} a_{25} a_{11}-b_{32} a_{44} a_{25} a_{66}+b_{32} a_{44} a_{21} a_{15}-b_{32} a_{25} a_{66} a_{77}$ $-b_{32} a_{25} a_{66} a_{88}-b_{32} a_{44} a_{25} a_{88}-b_{32} a_{33} a_{25} a_{77}-b_{32} a_{33} a_{25} a_{11}-b_{32} a_{33} a_{25} a_{66}+b_{32} a_{33} a_{21} a_{15}-b_{32} a_{33} a_{25} a_{88}-b_{32} a_{33} a_{44} a_{25}$ $-b_{32} a_{25} a_{77} a_{88}$

$E_{4}=-b_{32} a_{44} a_{25} a_{76} a_{67}+b_{32} a_{44} a_{25} a_{11} a_{88}+b_{32} a_{44} a_{25} a_{11} a_{77}-b_{32} a_{44} a_{61} a_{25} a_{16}-b_{32} a_{44} a_{81} a_{25} a_{18}+b_{32} a_{44} a_{25} a_{11} a_{66}$ $+b_{32} a_{33} a_{25} a_{77} a_{88}+b_{32} a_{33} a_{25} a_{66} a_{88}+b_{32} a_{33} a_{25} a_{66} a_{77}-b_{32} a_{33} a_{21} a_{15} a_{88}-b_{32} a_{33} a_{21} a_{15} a_{77}-b_{32} a_{33} a_{21} a_{15} a_{66}$ $+b_{32} a_{33} a_{21} a_{65} a_{16}+b_{32} a_{33} a_{21} a_{85} a_{18}-b_{32} a_{33} a_{25} a_{76} a_{67}+b_{32} a_{33} a_{25} a_{11} a_{88}+b_{32} a_{33} a_{25} a_{11} a_{77}-b_{32} a_{33} a_{61} a_{25} a_{16}$ $-b_{32} a_{33} a_{81} a_{25} a_{18}+b_{32} a_{33} a_{25} a_{11} a_{66}+b_{32} a_{25} a_{11} a_{77} a_{88}+b_{32} a_{33} a_{44} a_{25} a_{11}+b_{32} a_{33} a_{44} a_{25} a_{66}-b_{32} a_{33} a_{44} a_{21} a_{15}$ $+b_{32} a_{33} a_{44} a_{25} a_{88}-b_{32} a_{25} a_{76} a_{67} a_{88}+b_{32} a_{25} a_{86} a_{67} a_{78}+b_{32} a_{33} a_{44} a_{25} a_{77}+b_{32} a_{25} a_{11} a_{66} a_{88}+b_{32} a_{21} a_{15} a_{76} a_{67}$ $-b_{32} a_{21} a_{75} a_{16} a_{67}-b_{32} a_{21} a_{65} a_{86} a_{18}+b_{32} a_{21} a_{65} a_{16} a_{77}-b_{32} a_{61} a_{25} a_{16} a_{88}-b_{32} a_{61} a_{25} a_{16} a_{77}+b_{32} a_{21} a_{85} a_{18} a_{77}$ $+b_{32} a_{25} a_{11} a_{66} a_{77}-b_{32} a_{25} a_{11} a_{76} a_{67}-b_{32} a_{21} a_{15} a_{66} a_{77}-b_{32} a_{21} a_{15} a_{66} a_{88}-b_{32} a_{21} a_{15} a_{77} a_{88}+b_{32} a_{44} a_{25} a_{77} a_{88}$ $+b_{32} a_{44} a_{25} a_{66} a_{88}+b_{32} a_{44} a_{25} a_{66} a_{77}-b_{32} a_{81} a_{25} a_{18} a_{66}-b_{32} a_{44} a_{21} a_{15} a_{88}-b_{32} a_{44} a_{21} a_{15} a_{77}-b_{32} a_{44} a_{21} a_{15} a_{66}$ 
$-b_{32} a_{81} a_{25} a_{18} a_{77}+b_{32} a_{21} a_{65} a_{16} a_{88}+b_{32} a_{21} a_{85} a_{18} a_{66}+b_{32} a_{61} a_{25} a_{86} a_{18}+b_{32} a_{25} a_{66} a_{77} a_{88}+b_{32} a_{44} a_{21} a_{65} a_{16}$ $+b_{32} a_{44} a_{21} a_{85} a_{18}$,

$E_{5}=-b_{32} a_{33} a_{21} a_{85} a_{18} a_{77}+b_{32} a_{33} a_{21} a_{15} a_{77} a_{88}+b_{32} a_{81} a_{25} a_{16} a_{67} a_{78}+b_{32} a_{44} a_{25} a_{11} a_{76} a_{67}-b_{32} a_{44} a_{25} a_{11} a_{66} a_{77}$ $-b_{32} a_{21} a_{75} a_{86} a_{18} a_{67}-b_{32} a_{81} a_{25} a_{76} a_{18} a_{67}+b_{32} a_{33} a_{61} a_{25} a_{16} a_{77}+b_{32} a_{33} a_{25} a_{11} a_{76} a_{67}-b_{32} a_{33} a_{25} a_{11} a_{66} a_{77}$ $+b_{32} a_{44} a_{25} a_{76} a_{67} a_{88}+b_{32} a_{44} a_{21} a_{75} a_{16} a_{67}-b_{32} a_{44} a_{21} a_{15} a_{76} a_{67}-b_{32} a_{44} a_{25} a_{11} a_{66} a_{88}+b_{32} a_{44} a_{21} a_{15} a_{66} a_{77}$ $-b_{32} a_{33} a_{44} a_{25} a_{66} a_{88}+b_{32} a_{21} a_{15} a_{66} a_{77} a_{88}+b_{32} a_{21} a_{15} a_{86} a_{67} a_{78}-b_{32} a_{44} a_{25} a_{86} a_{67} a_{78}-b_{32} a_{21} a_{85} a_{18} a_{66} a_{77}$ $+b_{32} a_{21} a_{85} a_{76} a_{18} a_{67}-b_{32} a_{21} a_{65} a_{16} a_{77} a_{88}-b_{32} a_{25} a_{11} a_{86} a_{67} a_{78}+b_{32} a_{61} a_{25} a_{16} a_{77} a_{88}-b_{32} a_{21} a_{15} a_{76} a_{67} a_{88}$ $+b_{32} a_{21} a_{65} a_{86} a_{18} a_{77}+b_{32} a_{21} a_{75} a_{16} a_{67} a_{88}+b_{32} a_{25} a_{11} a_{76} a_{67} a_{88}-b_{32} a_{33} a_{21} a_{65} a_{16} a_{88}-b_{32} a_{33} a_{25} a_{66} a_{77} a_{88}$ $-b_{32} a_{33} a_{61} a_{25} a_{86} a_{18}-b_{32} a_{44} a_{21} a_{85} a_{18} a_{77}+b_{32} a_{44} a_{61} a_{25} a_{16} a_{77}+b_{32} a_{44} a_{21} a_{15} a_{66} a_{88}-b_{32} a_{44} a_{21} a_{65} a_{16} a_{77}$ $+b_{32} a_{44} a_{21} a_{65} a_{86} a_{18}-b_{32} a_{33} a_{44} a_{25} a_{77} a_{88}+b_{32} a_{81} a_{25} a_{18} a_{66} a_{77}+b_{32} a_{33} a_{21} a_{65} a_{86} a_{18}+b_{32} a_{44} a_{21} a_{15} a_{77} a_{88}$ $+b_{32} a_{33} a_{81} a_{25} a_{18} a_{66}-b_{32} a_{33} a_{25} a_{86} a_{67} a_{78}-b_{32} a_{33} a_{21} a_{65} a_{16} a_{77}+b_{32} a_{33} a_{44} a_{21} a_{15} a_{77}+b_{32} a_{33} a_{44} a_{21} a_{15} a_{88}$ $-b_{32} a_{33} a_{44} a_{25} a_{66} a_{77}+b_{32} a_{33} a_{21} a_{15} a_{66} a_{77}+b_{32} a_{33} a_{21} a_{15} a_{66} a_{88}-b_{32} a_{61} a_{25} a_{86} a_{18} a_{77}+b_{32} a_{33} a_{44} a_{61} a_{25} a_{16}$ $-b_{32} a_{33} a_{44} a_{25} a_{11} a_{77}-b_{32} a_{21} a_{85} a_{16} a_{67} a_{78}-b_{32} a_{25} a_{11} a_{66} a_{77} a_{88}+b_{32} a_{33} a_{44} a_{25} a_{76} a_{67}-b_{32} a_{33} a_{44} a_{21} a_{85} a_{18}$ $-b_{32} a_{33} a_{44} a_{21} a_{65} a_{16}+b_{32} a_{33} a_{44} a_{21} a_{15} a_{66}-b_{32} a_{33} a_{44} a_{25} a_{11} a_{88}-b_{32} a_{33} a_{44} a_{25} a_{11} a_{66}+b_{32} a_{33} a_{44} a_{81} a_{25} a_{18}$ $+b_{32} a_{44} a_{61} a_{25} a_{16} a_{88}+b_{32} a_{44} a_{81} a_{25} a_{18} a_{66}+b_{32} a_{44} a_{81} a_{25} a_{18} a_{77}-b_{32} a_{44} a_{25} a_{11} a_{77} a_{88}-b_{32} a_{33} a_{25} a_{11} a_{77} a_{88}$ $+b_{32} a_{33} a_{25} a_{76} a_{67} a_{88}+b_{32} a_{33} a_{81} a_{25} a_{18} a_{77}-b_{32} a_{33} a_{21} a_{85} a_{18} a_{66}-b_{32} a_{44} a_{25} a_{66} a_{77} a_{88}-b_{32} a_{44} a_{61} a_{25} a_{86} a_{18}$ $-b_{32} a_{44} a_{21} a_{85} a_{18} a_{66}-b_{32} a_{44} a_{21} a_{65} a_{16} a_{88}+b_{32} a_{33} a_{21} a_{75} a_{16} a_{67}-b_{32} a_{33} a_{21} a_{15} a_{76} a_{67}-b_{32} a_{33} a_{25} a_{11} a_{66} a_{88}$ $+b_{32} a_{33} a_{61} a_{25} a_{16} a_{88}$,

$$
\begin{aligned}
& E_{6}=-b_{32} a_{44} a_{21} a_{85} a_{76} a_{18} a_{67}-b_{32} a_{33} a_{25} a_{11} a_{76} a_{67} a_{88}-b_{32} a_{33} a_{44} a_{25} a_{76} a_{67} a_{88}+b_{32} a_{33} a_{44} a_{25} a_{11} a_{66} a_{77} \\
& +b_{32} a_{33} a_{21} a_{15} a_{76} a_{67} a_{88}-b_{32} a_{44} a_{21} a_{15} a_{66} a_{77} a_{88}+b_{32} a_{44} a_{25} a_{11} a_{66} a_{77} a_{88}+b_{32} a_{44} a_{21} a_{65} a_{16} a_{77} a_{88} \\
& +b_{32} a_{33} a_{44} a_{21} a_{85} a_{18} a_{77}-b_{32} a_{33} a_{21} a_{75} a_{16} a_{67} a_{88}+b_{32} a_{33} a_{44} a_{25} a_{66} a_{77} a_{88}-b_{32} a_{33} a_{21} a_{85} a_{76} a_{18} a_{67} \\
& -b_{32} a_{33} a_{81} a_{25} a_{18} a_{66} a_{77}-b_{32} a_{33} a_{44} a_{81} a_{25} a_{18} a_{77}-b_{32} a_{33} a_{21} a_{15} a_{66} a_{77} a_{88}+b_{32} a_{33} a_{44} a_{25} a_{11} a_{77} a_{88} \\
& +b_{32} a_{44} a_{21} a_{15} a_{76} a_{67} a_{88}+b_{32} a_{44} a_{21} a_{85} a_{18} a_{66} a_{77}+b_{32} a_{33} a_{44} a_{21} a_{65} a_{16} a_{77}+b_{32} a_{33} a_{44} a_{21} a_{65} a_{16} a_{88} \\
& -b_{32} a_{33} a_{21} a_{15} a_{86} a_{67} a_{78}-b_{32} a_{44} a_{25} a_{11} a_{76} a_{67} a_{88}+b_{32} a_{33} a_{44} a_{61} a_{25} a_{86} a_{18}+b_{32} a_{33} a_{25} a_{11} a_{66} a_{77} a_{88} \\
& +b_{32} a_{44} a_{25} a_{11} a_{86} a_{67} a_{78}-b_{32} a_{44} a_{81} a_{25} a_{16} a_{67} a_{78}-b_{32} a_{33} a_{44} a_{61} a_{25} a_{16} a_{77}+b_{32} a_{33} a_{21} a_{75} a_{86} a_{18} a_{67} \\
& +b_{32} a_{33} a_{44} a_{21} a_{85} a_{18} a_{66}+b_{32} a_{33} a_{44} a_{25} a_{11} a_{66} a_{88}-b_{32} a_{44} a_{21} a_{65} a_{86} a_{18} a_{77}-b_{32} a_{33} a_{44} a_{61} a_{25} a_{16} a_{88} \\
& -b_{32} a_{44} a_{21} a_{15} a_{86} a_{67} a_{78}-b_{32} a_{33} a_{44} a_{21} a_{75} a_{16} a_{67}+b_{32} a_{33} a_{44} a_{25} a_{86} a_{67} a_{78}+b_{32} a_{44} a_{61} a_{25} a_{86} a_{18} a_{77} \\
& -b_{32} a_{44} a_{21} a_{75} a_{16} a_{67} a_{88}-b_{32} a_{33} a_{81} a_{25} a_{16} a_{67} a_{78}-b_{32} a_{33} a_{61} a_{25} a_{16} a_{77} a_{88}+b_{32} a_{33} a_{44} a_{21} a_{15} a_{76} a_{67} \\
& -b_{32} a_{33} a_{44} a_{81} a_{25} a_{18} a_{66}-b_{32} a_{33} a_{44} a_{21} a_{15} a_{77} a_{88}-b_{32} a_{44} a_{81} a_{25} a_{18} a_{66} a_{77}+b_{32} a_{33} a_{81} a_{25} a_{76} a_{18} a_{67} \\
& -b_{32} a_{44} a_{61} a_{25} a_{16} a_{77} a_{88}+b_{32} a_{44} a_{21} a_{85} a_{16} a_{67} a_{78}-b_{32} a_{33} a_{21} a_{65} a_{86} a_{18} a_{77}+b_{32} a_{33} a_{61} a_{25} a_{86} a_{18} a_{77} \\
& -b_{32} a_{33} a_{44} a_{25} a_{11} a_{76} a_{67}-b_{32} a_{33} a_{44} a_{21} a_{15} a_{66} a_{77}+b_{32} a_{33} a_{21} a_{85} a_{18} a_{66} a_{77}+b_{32} a_{33} a_{21} a_{85} a_{16} a_{67} a_{78} \\
& -b_{32} a_{33} a_{44} a_{21} a_{65} a_{86} a_{18}-b_{32} a_{33} a_{44} a_{21} a_{15} a_{66} a_{88}+b_{32} a_{44} a_{21} a_{75} a_{86} a_{18} a_{67}+b_{32} a_{44} a_{81} a_{25} a_{76} a_{18} a_{67} \\
& +b_{32} a_{33} a_{25} a_{11} a_{86} a_{67} a_{78}+b_{32} a_{33} a_{21} a_{65} a_{16} a_{77} a_{88} \text {, } \\
& E_{7}=b_{32} a_{33} a_{44} a_{61} a_{25} a_{16} a_{77} a_{88}-b_{32} a_{33} a_{44} a_{21} a_{85} a_{18} a_{66} a_{77}+b_{32} a_{33} a_{44} a_{21} a_{85} a_{76} a_{18} a_{67} \\
& +b_{32} a_{33} a_{44} a_{25} a_{11} a_{76} a_{67} a_{88}-b_{32} a_{33} a_{44} a_{21} a_{75} a_{86} a_{18} a_{67}-b_{32} a_{33} a_{44} a_{21} a_{65} a_{16} a_{77} a_{88} \\
& +b_{32} a_{33} a_{44} a_{21} a_{15} a_{86} a_{67} a_{78}-b_{32} a_{33} a_{44} a_{81} a_{25} a_{76} a_{18} a_{67}-b_{32} a_{33} a_{44} a_{21} a_{15} a_{76} a_{67} a_{88} \\
& -b_{32} a_{33} a_{44} a_{25} a_{11} a_{66} a_{77} a_{88}-b_{32} a_{33} a_{44} a_{61} a_{25} a_{86} a_{18} a_{77}-b_{32} a_{33} a_{44} a_{21} a_{85} a_{16} a_{67} a_{78} \\
& +b_{32} a_{33} a_{44} a_{21} a_{75} a_{16} a_{67} a_{88}-b_{32} a_{33} a_{44} a_{25} a_{11} a_{86} a_{67} a_{78}+b_{32} a_{33} a_{44} a_{21} a_{15} a_{66} a_{77} a_{88} \\
& +b_{32} a_{33} a_{44} a_{81} a_{25} a_{16} a_{67} a_{78}+b_{32} a_{33} a_{44} a_{21} a_{65} a_{86} a_{18} a_{77}+b_{32} a_{33} a_{44} a_{81} a_{25} a_{18} a_{66} a_{77} \text {, }
\end{aligned}
$$




\section{Conflicts of Interest}

The authors declare that there are no conflicts of interest.

\section{Acknowledgments}

This work is supported by National Natural Science Foundation of China (11772291, 11572278, and 11572084) and Innovative Research Team in University of Henan Province (13IRTSTHN019).

\section{References}

[1] J. Shen, Z. Liu, W. Zheng, F. Xu, and L. Chen, "Oscillatory dynamics in a simple gene regulatory network mediated by small RNAs," Physica A. Statistical Mechanics and its Applications, vol. 388, no. 14, pp. 2995-3000, 2009.

[2] X. Yang, M. Feng, X. Jiang et al., "miR-449a and miR-449b are direct transcriptional targets of E2F1 and negatively regulate pRb-E2F1 activity through a feedback loop by targeting CDK6 and CDC25A," Genes \& Development, vol. 23, no. 20, pp. 23882393, 2009.

[3] T. Bou Kheir, E. Futoma-Kazmierczak, A. Jacobsen et al., "miR449 inhibits cell proliferation and is down-regulated in gastric cancer," Molecular Cancer, vol. 10, article 29, 2011.

[4] E. Khav, Visualing an Rb-E2F cellular switch that controls cell oliferation [Dissertation, thesis], Academic Dissertation, The University of Arizona, 2013.

[5] J. White, E. Stead, R. Faast, S. Conn, P. Cartwright, and S. Dalton, "Developmental activation of the Rb-E2F pathway and establishment of cell cycle-regulated cyclin-dependent kinase activity during embryonic stem cell differentiation," Molecular Biology of the Cell, vol. 16, no. 4, pp. 2018-2027, 2005.

[6] J. R. Nevins, G. Leone, J. DeGregori, and L. Jakoi, "Role of the Rb/E2F pathway in cell growth control," Journal of Cellular Physiology, vol. 173, no. 2, pp. 233-236, 1997.

[7] J. R. Nevins, "The Rb/E2F pathway and cancer," Human Molecular Genetics, vol. 10, no. 7, pp. 699-703, 2001.

[8] L. Hangnoh, Regulation of differentiation-specific genes by the Drosophila $R B, E 2 F$, and Myb-interacting proteins complex (dREAM [Dissertation, thesis], Academic Dissertation, The State University of New Jersey, 2011.

[9] D. Hanahan and R. A. Weinberg, "The hallmarks of cancer," Cell, vol. 100, no. 1, pp. 57-70, 2000.

[10] R. C. Sears and J. R. Nevins, "Signaling networks that link cell proliferation and cell fate," Journal of Biological Chemistry, vol. 277, no. 14, pp. 11617-11620, 2002.

[11] N. Ghanem, M. G. Andrusiak, D. Svoboda et al., "The Rb/E2F pathway modulates neurogenesis through direct regulation of the Dlx1/Dlx2 bigene cluster," Journal of Neuroscience, vol. 32, no. 24, pp. 8219-8230, 2012.

[12] F. Yan, H. Liu, J. Hao, and Z. Liu, "Dynamical Behaviors of Rb-E2F Pathway Including Negative Feedback Loops Involving miR449," PLoS ONE, vol. 7, no. 9, Article ID e43908, 2012.

[13] M. N. Obeyesekere, S. O. Zimmerman, E. S. Tecarro, and G. Auchmuty, "A model of cell cycle behavior dominated by kinetics of a pathway stimulated by growth factors," Bulletin of Mathematical Biology, vol. 61, no. 5, pp. 917-934, 1999.

[14] G. Yao, T. J. Lee, S. Mori, J. R. Nevins, and L. You, "A bistable RbE2F switch underlies the restriction point," Nature Cell Biology, vol. 10, no. 4, pp. 476-482, 2008.
[15] H. Chen, P. A. Mundra, L. N. Zhao, F. Lin, and J. Zheng, "Highly sensitive inference of time-delayed gene regulation by network deconvolution," BMC Systems Biology, vol. 8, no. 4, article no. S6, 2014.

[16] J.-P. Richard, "Time-delay systems: an overview of some recent advances and open problems," Automatica. A Journal of IFAC, the International Federation of Automatic Control, vol. 39, no. 10, pp. 1667-1694, 2003.

[17] C. Y. Ko, M. B. Liu, Z. Song, Z. Qu, and J. N. Weiss, "Multiscale Determinants of Delayed Afterdepolarization Amplitude in Cardiac Tissue," Biophysical Journal, vol. 112, no. 9, pp. 19491961, 2017.

[18] L. Mier-y-Tern-Romero, M. Silber, and V. Hatzimanikatis, "The origins of time-delay in template biopolymerization processes," PLoS Computational Biology, vol. 6, no. 4, e1000726, 15 pages, 2010.

[19] Q. Zheng, Z. Wang, and J. Shen, "Pattern dynamics of networkorganized system with cross-diffusion," Chinese Physics B, vol. 26, no. 2, p. 020501, 2017.

[20] Q. Zheng and J. Shen, "Dynamics and pattern formation in a cancer network with diffusion," Communications in Nonlinear Science and Numerical Simulation, vol. 27, no. 1-3, pp. 93-109, 2015.

[21] Y. Xu, Y.-N. Zhu, J. W. Shen, and J. B. Su, "Switch dynamics for stochastic model of genetic toggle switch," Physica A: Statistical Mechanics and Its Applications, vol. 416, pp. 461-466, 2014.

[22] Q. Zheng and J. Shen, "Pattern formation in the FitzHughNagumo model," Computers \& Mathematics with Applications, vol. 70, no. 5, pp. 1082-1097, 2015.

[23] Q. Zheng and J. Shen, "Turing instability in a gene network with cross-diffusion," Nonlinear Dynamics. An International Journal of Nonlinear Dynamics and Chaos in Engineering Systems, vol. 78, no. 2, pp. 1301-1310, 2014.

[24] Q. Zheng and J. Shen, "Bifurcations and dynamics of cancer signaling network regulated by MicroRNA," Discrete Dynamics in Nature and Society, vol. 2013, Article ID 176956, 2013.

[25] G. Orosz, J. Moehlis, and R. M. Murray, "Controlling biological networks by time-delayed signals," Philosophical Transactions of the Royal Society of London. Series A. Mathematical, Physical and Engineering Sciences, vol. 368, no. 1911, pp. 439-454, 2010.

[26] Z. Song, Z. Qu, and A. Karma, "Stochastic initiation and termination of calcium-mediated triggered activity in cardiac myocytes," Proceedings of the National Academy of Sciences, vol. 114, no. 3, pp. E270-E279, 2017.

[27] P. Zoppoli, S. Morganella, and M. Ceccarelli, "TimedelayARACNE: reverse engineering of gene networks from timecourse data by an information theoretic approach," BMC Bioinformatics, vol. 11, no. 1, article 154, 2010.

[28] Y. Li and A. Ngom, "The max-min high-order dynamic Bayesian network learning for identifying gene regulatory networks from time-series microarray data," in Proceedings of the 10th Annual IEEE Symposium on Computational Intelligence in Bioinformatics and Computational Biology, CIBCB 2013 - 2013 IEEE Symposium Series on Computational Intelligence, SSCI 2013, pp. 83-90, April 2013.

[29] Y. Cao and P. M. Frank, "Analysis and synthesis of nonlinear time-delay systems via fuzzy control approach," IEEE Transactions on Fuzzy Systems, vol. 8, no. 2, pp. 200-211, 2000.

[30] Z. Song, C. Y. Ko, M. Nivala, J. N. Weiss, and Z. Qu, "Complex darly and delayed afterdepolarization dynamics caused 
by voltage-calcium coupling in cardiac myocytes," Biophysical Journal, vol. 108, no. 8, pp. 261A-262A, 2015.

[31] C. Y. Ko, Z. Song, Z. Qu, and J. N. Weiss, "Multiscale Consequences of Spontaneous Calcium Release on Cardiac Delayed Afterdepolarizations," Biophysical Journal, vol. 108, no. 2, p. 264a, 2015. 




Advances in

Operations Research

vatersals

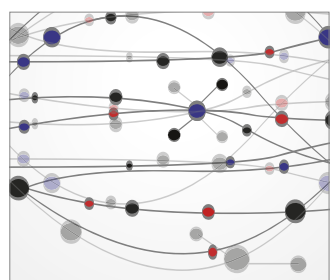

\section{The Scientific} World Journal
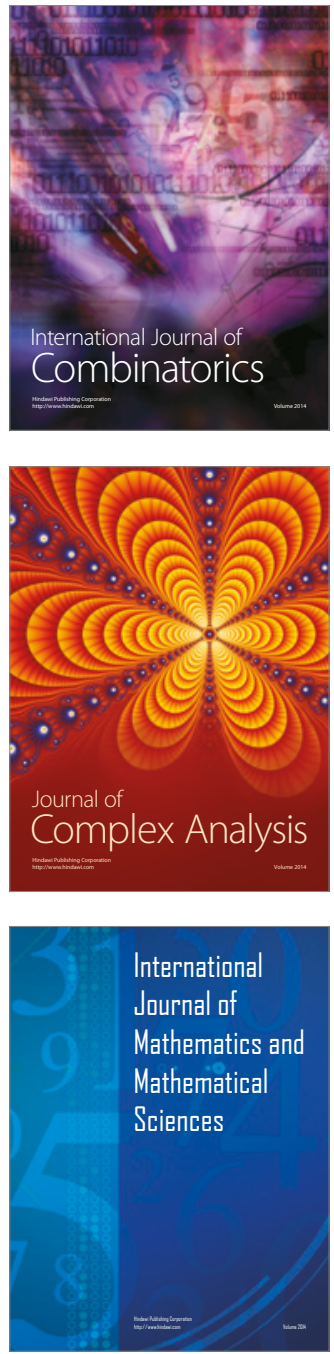
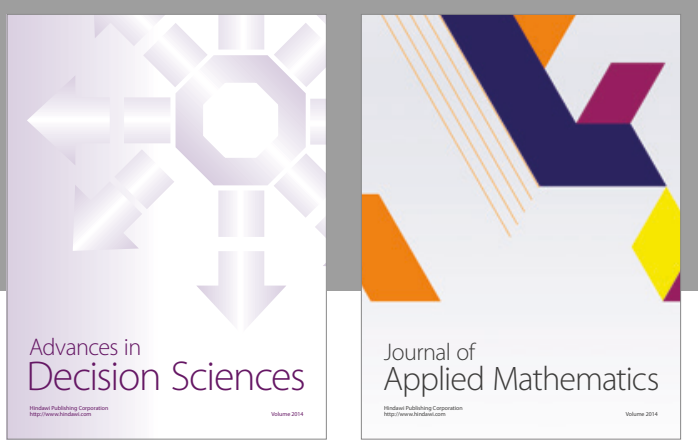

Algebra

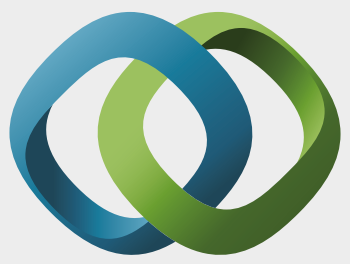

\section{Hindawi}

Submit your manuscripts at

https://www.hindawi.com
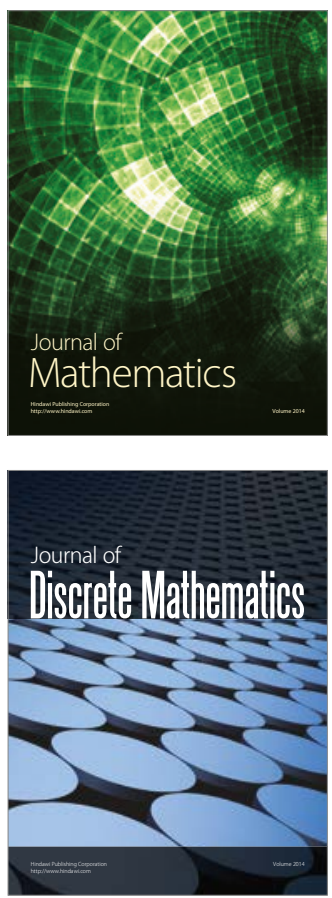

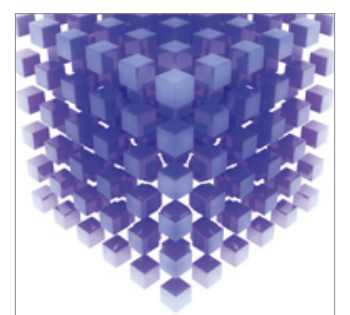

Mathematical Problems in Engineering
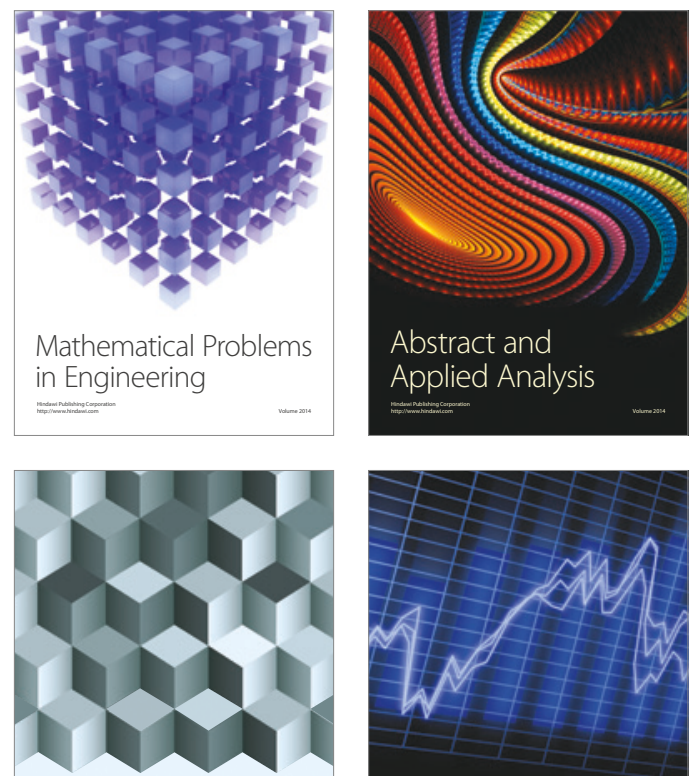

Journal of

Function Spaces

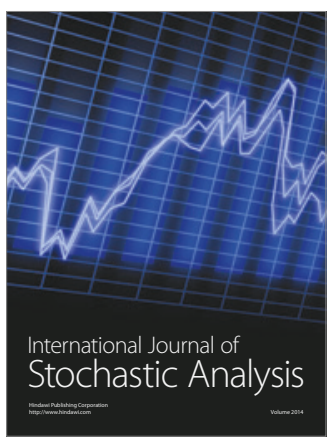

Probability and Statistics
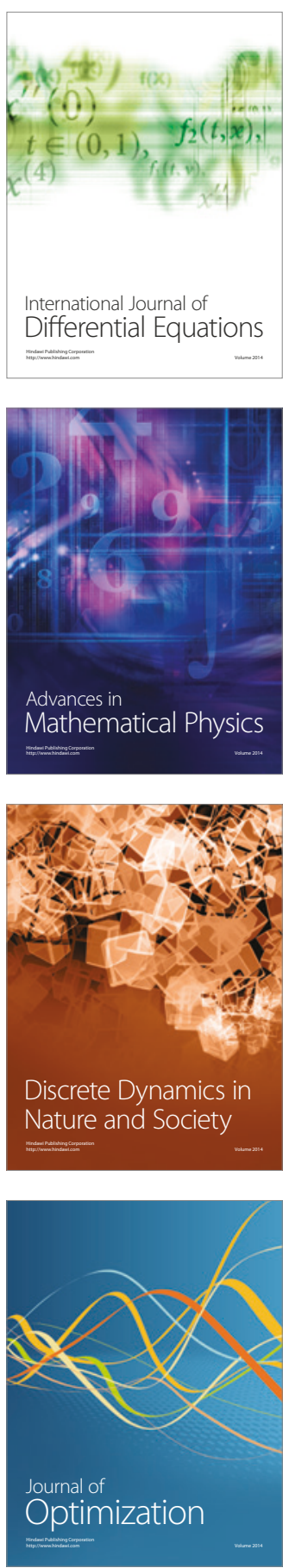